\title{
51st ISCEV international symposium abstract issue
}

Springer-Verlag Berlin Heidelberg 2013

\section{Editorial note: The abstracts in this issue have been edited and re-set to a standard format. We have made every effort to respect the intended meaning of the originals and apologise for any inadvertent misinterpretation.}

\section{Oral Abstracts}

\section{William Dawson Memorial Lecture: Melatonin modulates excitatory and inhibitory signal Transmission in the retina}

\section{Professor Yang XL}

Institute of Neurobiology, Institutes of Brain Sciences and State Key Laboratory of Medical Neurobiology, Fudan University, 138 Yixueyuan Rd. Shanghai 200032, China

Melatonin, a primary hormone originally discovered in the pineal gland, is involved in the regulation of a variety of physiological processes through activation of specific G-protein coupled receptors (MT1 and MT2). In the retina melatonin is released by photoreceptors in a circadian manner, and its receptors are abundantly expressed in various types of neurons. In recent years, our group has made an extensive study of melatonin-induced modulation of the activity of retinal neurons, using multidisciplinary approaches.

In the outer retina we studied how melatonin modulates excitatory transmission at horizontal and bipolar cell levels in carp retina. Melatonin potentiates glutamate-induced whole cell currents from isolated cone-driven, but not rod-driven, horizontal cells by increasing the efficacy and apparent affinity of the glutamate receptors. The effects of melatonin are mediated by the MT1 receptor, and the activation of this receptor causes a decrease of the intracellular concentration of cGMP. Consistently, melatonin depolarizes the membrane potential of these cells and reduces their light responses, recorded intracellularly in isolated, superfused carp retina. At the bipolar cell level, melatonin activates the MT2 receptor on rod-driven ON type cells, thus increasing intracellular cGMP levels through an inhibition of phosphodiesterase. Physiologically, melatonin potentiates responses of these rod-driven bipolar cells to simulated light flashes, which is mediated by cGMP-dependent kinase, and increases the amplitude of the scotopic electroretinographic b-wave, a reflection of rod-driven ON type bipolar cell activity. These results suggest that in the outer retina melatonin, being at a higher level at night, suppresses cone signal transfer to horizontal cells, but improves the signal/noise ratio for rod signals by enhancing signal transfer from rods to bipolar cells.

In the inner retina, we studied melatonin-induced modulation of inhibitory transmission to ganglion cells. Melatonin potentiates whole cell glycine currents of isolated ganglion cells by activating the MT2 receptor. A distinct phosphatidylcholine (PC)-phosphokinase $\mathrm{C}$ (PLC)/PKC signaling pathway, following the activation of MT2 receptors, is most likely responsible for the melatonin effects. Consistent with this, in retinal slice preparations melatonin potentiates light-evoked glycine receptor-mediated inhibitory postsynaptic currents in ganglion cells. These results suggest that melatonin may help animals to detect positive or negative contrast in night vision by modulating inhibitory signals largely mediated by glycinergic amacrine cells in the inner retina.

Taken together, melatonin may contribute to detect dim light signals and/or contrast in night vision by differentially modulating rod versus cone signal transfer in the outer retina and/or enhancing inhibitory signals in the inner retina.

\section{Invited Lecture 1: Transplantation of retinal progenitor cells into the subretinal space of retinitis pigmentosa patients: one year follow-up study}

\section{Professor Zheng Qin Yin}

Southwest Hospital/Southwest Eye Hospital, Third Military Medical University, Chongqing, China 
Retinitis pigmentosa (RP) is the most common inherited retinal degenerative disease and currently has no treatment. Preclinical studies have demonstrated efficacy in photoreceptor precursor transplantation in animal models of RP. In a phase I clinical trial, we assessed the safety of retinal cell transplantation in RP patients. Neuroretinal cells (NRCs) from 12 to 16 week human fetuses or retinal progenitor cells (RPCs) further purified from NRCs were transplanted into the subretinal space of RP patients. In the RPC transplanted group of 6 patients, there was no clinical evidence of immunological rejection and there was an improvement in visual acuity at 3 and 6 months follow-up $(P<0.05)$ over 12 months. In the NRC transplanted group, three of four cases had signs of immune rejection and none of the patients experienced visual improvement. This study shows the potential efficacy of human stem cell transplantation and provides the basis for future transplantation studies in RP and other retinal degenerative diseases.

\section{Invited Lecture 2: The multiple evaluation of visual electrophysiology in glaucoma}

\section{Professor LezhengWu}

Zhongshan Ophthalmic Center, Sun Yat-Sen University, Guangzhou, China

Glaucoma is still a serious eye problem that can lead to blindness. Loss of visual function is an important characteristic of damage to the optic nerve in glaucoma. Routine visual field testing is as one of the subjective examinations for evaluation of visual function, but multiple visual electrophysiological measurements may be favourable to assess objectively visual function in glaucoma. An abnormality in pattern ERG (PERG) may be earlier than visual field changes by several years in glaucoma. With normal retinal nerve fiber thickness in OCT in high tension glaucoma the PERG may be abnormal. The total score of mfVEP and the asymmetry of amplitudes in mfERG were useful to differentiate the normal tension glaucoma and the progress of glaucoma. The photopic negative potential $(\mathrm{PhNR})$ which is becoming more popular may play an effective role in the assessment of varied visual functions in glaucoma. It is important that multiple evaluations by visual electrophysiology can benefit the differentiation of suspect glaucoma / normal tension glaucoma and are valuable in the assessment of the early variations in visual function as well as the progress and severity in glaucoma.

\section{Oral Session 1: Retinal degeneration I}

\section{OS1: Oral paper 1}

Clinical and molecular analysis of Stargardt disease with preserved foveal structure and function

Fujinami $K^{1,5}$, Sergouniotis $\mathbf{P I}^{4,5}$, Tsunoda $K^{1}$, Tsubota $K^{3}$, Robson $A_{G}^{4,5}$, Moore $A^{4,5}$, Michaelides $M^{4,5}$, Webster $A^{4,5}$, Holder GE ${ }^{4,5}$

${ }^{1}$ Laboratory of Visual Physiology, National Institute of Sensory Organs, Tokyo, Japan, ${ }^{2}$ National Hospital Organization, Tokyo Medical Center, Tokyo, Japan,
${ }^{3}$ Keio University, School of Medicine, Tokyo, Japan, ${ }^{4} \mathrm{UCL}$ Institute of Ophthalmology, London, UK, ${ }^{5}$ Moorfields Eye Hospital, London, UK

\section{Purpose}

To describe a cohort of patients with Stargardt disease (STGD) who show a foveal sparing phenotype.

Methods

The foveal sparing phenotype was defined as foveal preservation on autofluorescence (AF) imaging, despite a retinopathy otherwise consistent with STGD. Forty such individuals were ascertained from a survey of AF images of 438 patients with STGD. A detailed history and ophthalmic examination was undertaken. Investigations included colour fundus photography, AF imaging, spectral domain optical coherence tomography (SD-OCT), and PERG and full-field ERGs that incorporated the ISCEV standards. All main components of the ERG and the PERG were used to classify patients into three groups (Lois et al. 1999; Fujinami et al. 2013); dysfunction confined to the macula (group 1), macular and generalised cone dysfunction (group 2) and macular and generalised cone and rod dysfunction (group 3). Mutation screening of ABCA4 was performed with arrayed primer extension (APEX) technology (AsperBiotech). The molecular findings of patients with foveal sparing phenotype were compared to those of patients with STGD harbouring at least one disease-causing variant detected by APEX but no foveal sparing $(\mathrm{N}=140)$.

\section{Results}

The median age of onset and age at examination of 40 patients with the foveal sparing phenotype was 43.5 and 46.5 years, respectively. The median $\log$ MAR visual acuity was 0.18 . Twenty-two patients $(22 / 40,55 \%)$ had patchy parafoveal atrophy and flecks; 8 (20\%) had numerous flecks at the posterior pole without atrophy; $7(17.5 \%)$ had mottled retinal pigment epithelial changes; $2(5 \%)$ had multiple atrophic lesions, extending beyond the arcades; and $1(2.5 \%)$ had a bull's-eye appearance. The median central foveal thickness assessed with SD-OCT was $183.0 \mu \mathrm{m}(\mathrm{n}=33)$, with outer retinal tubulation observed in $15(45 \%)$. Twenty-two $(67 \%)$ of 33 subjects were in ERG group 1;3 $(9 \%)$ in ERG group 2; and $8(24 \%)$ in ERG group 3. PERG was normal in both eyes of 3 patients $(9 \%)$, abnormal in either eye of 16 subjects ( $48 \%$ ), and undetectable in either eye of 14 individuals (42\%). Diseasecausing variants in ABCA4 were found in 31 patients (31/40, $78 \%$ ). There was a significantly higher prevalence of the variant p.Arg2030Gln in the cohort with foveal sparing compared to the group with foveal atrophy $(6.45 \%$ vs $1.07 \%$-Fisher Exact test two-tailed $p=0.022$ ).

\section{Conclusion}

Foveal sparing STGD can occur in all three electrophysiological types of retinal dysfunction. The presence of two distinct phenotypes of STGD (foveal sparing and foveal atrophy) suggests that there may be more than one disease-mechanism in ABCA4-retinopathy. Reliably identifying patients with foveal sparing prospectively has important implications for the selection of patients for therapeutic trials.

\section{OS1: Oral paper 2}

Clinical and molecular analysis of macular dystrophy with preserved foveal structure and function 
Goto $S^{1,2}$, Fujinami $K^{2,5}$, Akahori $M^{2}$, Iwata $T^{3}$, Noda $T^{3}$, Miyake $\mathbf{Y}^{2}$, Tsunoda $\mathbf{K}^{2}$

${ }^{1}$ Osaka University Graduate School of Medicine, Osaka, Japan, ${ }^{2}$ Laboratory of Visual Physiology, National Institute of Sensory Organs, Tokyo, Japan, ${ }^{3}$ National Hospital Organization, Tokyo Medical Center, Tokyo, Japan, ${ }^{4}$ Keio University, School of Medicine, Tokyo, Japan, ${ }^{5} \mathrm{UCL}$ Institute of Ophthalmology, London, UK

\section{Purpose}

Patients with foveal sparing (FS) phenotype of Stargardt Disease present with late-onset and tend to have maintained visual acuity, with harbouring disease-causing variants in the ABCA4 gene (Rotenstreich et al. 2003; Fujinami et al. 2010, 2013; Westenengvan Haaften et al. 2012). However, it has been unknown whether the FS finding occurs in other macular dystrophies (non-ABCA4retinopathy). The present study describes the clinical and molecular characteristics of four Japanese patients with macular dystrophy (MD) who show the FS finding.

\section{Methods}

The FS finding was defined on autofluorescence (AF) imaging, as an apparent foveal preservation (larger than half of the disc diameter) surrounded by macular atrophic lesions of low/high AF signal. Four simplex cases with the FS finding despite a retinopathy otherwise consistent with MD were ascertained. Detailed ophthalmic examinations, including color fundus photography, AF imaging, and spectral domain optical coherence tomography (SD-OCT) were undertaken. Electrophysiological assessment was performed with ISCEV standard full-field (ff) ERGs, and focal macular ERG/mfERG. Mutation screening of ABCA4 was performed with arrayed primer extension technology (ABCA4 APEX; AsperBiotech).

\section{Results}

The mean age of onset and the mean age at the baseline examination of the four cases with FS-COD/CORD was $35.3 \mathrm{yrs}$ (range 20-46 yrs) and 45.0 yrs (range 38-56 yrs), with the mean follow-up interval being 5.3 yrs (range 3-7 yrs). Decimal visual acuity (VA) at baseline and follow-up was better than 0.5 in both eyes of three patients (patients 1,2,3) and patient 4 showed rapid VA reduction from 0.2 to 0.06 in the left eye during the 4 year follow-up interval. Fundus photography revealed a normal foveal appearance with patchy multiple atrophies at the para-fovea in three patients (patients 1,2,3) and a normal fovea surrounded by nummular atrophy at the macula in one subject (patient 4). AF imaging detected macular lesions of low/high AF signal surrounded by an annular enhancement of high AF signal extended to the disc in all patients. SD-OCT demonstrated a detectable continuous inner segment ellipsoid (ISe) band at the fovea in two patients (patients 1,3), and a disappearance of ISe at the entire macula in two subjects (patients 2, 4). ffERGs detected slightly reduced cone-mediated responses in two patients (patients 1,3) and an electronegative waveform of dark-adapted bright flash ERGs (DA 30.0) in two subjects (patients 2, 4). Focal macular ERGs/mfERGs were recorded in three patients and a preserved central foveal response was observed in one eye of three patients (patients 1, 3, 4). Molecular genetic screening identified one previously reported likely disease-causing variant (c.1699G $>$ A, p.V567 M) in one patient (patient 3), with no disease-causing variants found in three subjects (patients $1,2,4$ ).

\section{Conclusion}

MD with the FS finding was identified and associated with a later onset of disease, better VA ( $>0.5)$, and preserved foveal structure and function. Two patients showed generalized cone dysfunction and two subjects had an electronegative waveform in DA 30.0. One disease-causing ABCA4 variant was detected in only one patient, which implies other causative genes can be responsible for this unique phenotype.

\section{OS1: Oral paper 3}

Phenotype-genotype correlation in patients with occult macular dystrophy associated with heterozygous RP1L1 missense variants

Robson AG, Davidson AE, Sergouniotis PI, Mackay DS, Wright GA, Waseem NH, Moore AT, Plagnol V, Holder GE, Webster AR

Moorfields Eye Hospital and UCL Institute of Ophthalmology, London, UK

\section{Purpose}

To characterise the clinical and electrophysiological features of patients with occult maculopathy and heterozygous RP1L1 variants Methods

A cohort of twenty eight patients were ascertained with reduced visual acuity and normal fundi and a clinical history compatible with maculopathy. The coding regions and intron/ exon boundaries of RP1L1 were sequenced, excluding a repetitive transcript (amino acids 1312 to 2,160). Fundus autofluorescence and spectral domain OCT were performed. Electrophysiological testing included International-standard pattern and full-field ERGs in all and mfERGs in some. The segregation of any rare RP1L1 variants identified in probands were checked in asymptomatic relatives from 5 families.

\section{Results}

Ten of the 28 patients with occult maculopathy were found to harbour rare heterozygous RP1L1 missense variants. The mean age of the 10 patients was 49 years (SD 18 years; range 31-78) and all presented with visual acuity reduction in one (1 patient) or both eyes between the 1st-7th decade (mean and median 4th decade). Mean $\log$ MAR visual acuity in affected eyes was 0.6 (SD 0.21, range 0.3-1.0). PERG P50 and/or mfERGs were subnormal in 10 of 10 patients. Full-field ERGs were normal in 8 and revealed mild generalised cone system dysfunction in 2 individuals. Irregular foveal fundus autofluorescence was evident in 6 of 10 cases. Spectral domain OCT showed focal disruption of the inner segment ellipsoid band in 8 of 9 cases. A missense variant in RP1L1 identical to that found in probands was identified in 4 asymptomatic relatives (aged 29, 57, 67 and 73 years) with normal fundus autofluorescence and normal electrophysiology, including a parent from each of 2 families.

\section{Conclusions}

Patients with occult macular dystrophy and heterozygous missense variants in RP1L1 show a range of electrophysiological and structural abnormalities. Detailed examination of asymptomatic relatives carrying the same allele can be normal. The heterozygous RP1L1 variants act as a risk factor for the disorder rather than being a fully-penetrant gene. 


\section{OS1: Oral paper 4}

Unilateral RP: learning from a 30 year follow-up

Lachapelle $\mathbf{P}^{\mathbf{1}}$, Gauvin $\mathrm{M}^{\mathbf{1}}$, Dorfman $\mathrm{AL}^{\mathbf{1}}$, Racine $\mathbf{J}^{\mathbf{1}}$, Koenekoop RK ${ }^{1}$, Little JM' ${ }^{1}$, Hébert $\mathbf{H}^{2}$, Lina $\mathbf{J M}^{3,4}$

${ }^{1}$ Departments of Ophthalmology, Neurology and Neurosurgery, McGill University, Montreal Children's Hospital, Montreal, Canada, ${ }^{2}$ Department of Ophthalmology, Centre de recherche Laval Robert-Giffard, Université Laval, ${ }^{3}$ Department of electrical engineering, École de Technologie Supérieure, ${ }^{4}$ Centre de Recherches Mathématiques, Montréal, Canada

\section{Purpose}

A 29 year old woman presented with constricted visual field, attenuated retinal vasculature, pigmentary clumping and reduced ERG in OS only, suggesting a diagnosis of unilateral retinitis pigmentosa (RP). This patient was subsequently seen on 8 occasions over 30 years and, with time, the initially normal eye (OD) gradually showed signs of RP-like retinal degeneration. On last examination (2012) the patient was legally blind OU. The purpose of this study was to evaluate which clinical test (visual field, retinal fundus or ERG) was first to predict this fate.

\section{Method}

Data obtained from control subjects [visual field area $(\mathrm{N}=30)$, retinal vessels diameter $(\mathrm{N}=20)$, scotopic and photopic ERG amplitude $(\mathrm{N}=80)$ and time-frequency (discrete wavelet transform (DWT) measurements of photopic ERG $(\mathrm{N}=80)]$ were compared (Z-score statistics) with the results from the patient. Linear interpolation was used to estimate $p$-values between two consecutive tests.

\section{Results}

At the initial visit, all tests were significantly altered in OS $(p<0.05)$ and normal in OD $(p>0.05)$. Visual field areas and retinal vessel calibers in OD gradually reduced to reach statistical significance $(p<0.05) 20.2$ and 19.1 years after the first examination, respectively. The amplitude of the scotopic ERG in OD also reduced gradually and became significantly smaller than normal $(p<0.05) 8.5$ years after the initial visit. Similarly, the amplitude of the photopic b-wave reached significance $(p<0.05)$ after 15.5 years. In contrast, when the DWT was used to quantify the photopic b-wave, we were able to detect a significant change (in the Hölder exponent descriptor) after 6.9 years only $(p<0.05)$. Finally, of note, the rate of degeneration (based on the photopic ERGs) progressed significantly faster ( 5 times faster) in the initially normal eye.

\section{Conclusion}

Our results demonstrate that the first sign of pathology was observed after 20 years with the traditional clinical features (retinal fundus and visual field), while the ERG was significantly altered after 8 (rod function) and 15 years (cone function). The cone ERG changes were detected even faster (after 7 years compared to 15) when a sophisticated mathematical tool were used (DWT). Our results demonstrate the advantage of the DWT for early detection of significant alteration in retinal function.

Funding sources: CIHR and Réseau Vision of the FRSQ

\section{OS1: Oral paper 5}

ERGs in developmentally delayed children with failure to thrive: facilitating diagnosis of PMM2-CDG

Thompson $\mathrm{D}^{1}$, Russell-Eggitt $\mathbf{I}^{1}$, Allen $\mathrm{L}^{2}$, Liasis $\mathrm{A}^{\mathbf{1}}$, Jagle $\mathbf{H}^{2}$, Grunewald $\mathbf{S}^{3}$

${ }^{1}$ Clinical and Academic Department of Ophthalmology, Great

Ormond Street Hospital for Children, London, UK,

${ }^{2}$ Ophthalmology Department, Addenbrookes NHS Trust, Cambridge, UK, ${ }^{3}$ University Eye Clinic, Regensburg, Germany

\section{Purpose}

To refine the retinal phenotype associated with the congenital disorder of glycosylation PMM2-CDG caused by a lack of the enzyme phosphomannomutase.

\section{Methods}

The ERGs and clinical reports of 12 patients with genetically confirmed PMM2-CDG were reviewed retrospectively; age at diagnosis ranged from $5 \mathrm{~m}$ to 15 years. ERGs were recorded with corneal electrodes to International Society of Clinical Electrophysiology of Vision standards where possible, extended to include a wider range of flash strengths and prolonged on-off photopic stimulation, or in younger children using the GOSH skin ERG protocol with a hand held stimulator. Results

All patients presented in early infancy with hypotonia, 9/12 showed a failure to thrive and 6/12 patients had experienced acute crises in the early years requiring hospital admission. MRI signs of cerebellar hypoplasia were remarked in 10/12 patients and 9/12 patients showed nystagmoid eye movements, some vertical. At the time of last review the patients exhibited a wide range of clinical severity.

All patients showed a subnormal ERG at first test, carried out at ages ranging from $5 \mathrm{~m}$ to $15 \mathrm{yrs}$ of age. The ERGs showed a distinctive pattern of retinal on-pathway dysfunction, in the form of reduced b-wave amplitudes and preserved oscillatory potentials in 9/12 patients. The remaining 3/12 patients had severe photoreceptor dysfunction by the time of ERG recording, with markedly attenuated a- and b-wave amplitudes.

\section{Conclusions}

ERG features of on-pathway dysfunction are evident in early stages of PMM2-CDG and in mildly manifesting disease. This recognisable pattern can help establish diagnosis when an underlying metabolic cause of developmental delay is suspected in infancy or indeed in later childhood.

\section{Oral Session 2: Origins of electrophysiological signals}

\section{OS2: Oral paper 1}

ERGs originate in photoreceptors and intrinsically photosensitive retinal ganglion cells (ipRGCs)

Caiping $\mathrm{Hu}^{1}$, Slaughter $\mathrm{MM}^{2}$, Kwoon Y Wang ${ }^{2}$

${ }^{1}$ Zhenzhou Eye Hospital, Zhenzhou second Hospital, Henan, China, ${ }^{2}$ Department of Physiology and Biophysics 
at University at Buffalo, State University of New York, USA, ${ }^{3}$ Kellogg Eye Center, University of Michigan, USA

\section{Purpose}

About a decade ago, a new ganglion cell (RGC) type was discovered that specializes in functions such as the pupillary light reflex, photo entrainment of circadian rhythms, and photic regulation of hormone secretion. These novel RGCs express the photo pigment melanopsin and function as photoreceptors, and thus are called ganglion-cell photoreceptors or intrinsically photosensitive retinal ganglion cells (ipRGCs) (Berson et al. 2002; Hattar et al. 2002; Provencio et al. 1998). How the light responses of these new photoreceptors change understanding of the ERG has not been studied.

\section{Methods}

We did patch clamp on ipRGCs for whole-cell recording, using mice in which all five types of melanopsin ganglion cells were selectively labeled with enhanced green fluorescent protein (EGFP) (Ecker et al. 2010). The morphologies and intrinsically electrophysiological properties were explored. The typical light responses of ipRGCs were compared with those of retinal bipolar cells (from rod and cones).

\section{Results}

Our studies revealed five morphological types of melanopsinexpressing RGCs in mice, M1 through M5. The five cell types have different dendritic stratification levels, dendritic morphologies, soma sizes and dendritic areas. In addition to anatomical differences, some of the five ipRGC types have been shown to have different photoresponse characteristics, and intrinsic electrophysiological properties, suggesting they are likely to perform diverse physiological functions. In terms of intrinsic electrophysiology, M1, M2 and M3 include two subtypes according to their different $\mathrm{Ca}^{2+}$ channel expressions while M4 and M5 cells are homogeneous. All ipRGCs produced asynchronized responses in contrast to the synchronized ON/OFF responses in retinal bipolar cells. The ipRGCs contributed to the ERG waves more than photoreceptors following the flash stimuli.

\section{Conclusions}

A characterization of the electrophysiology of ipRGCs and bipolar cells explain the clinical ERGs at the cellular level. These results have suggested that these ipRGC types contribute to ERGs. Both photoreceptors share the ERG waves in displaying their special visual function.

\section{OS2: Oral paper 2}

\section{Exogenous zinc ions interrupt signal transmission from photoreceptors to bipolar cells and reduce retinal TRPM1 expression in mouse}

\section{Bo Lei, Yu Liu, Tao Lin, Hongxia Yang}

Department of Ophthalmology, the First Affiliated Hospital of Chongqing Medical University and Chongqing Key Laboratory of Ophthalmology, Chongqing Eye Institute, Chongqing, China

\section{Purpose}

To investigate whether zinc ions affect visual signal transmission and transient receptor potential cation channel subfamily M member 1 (TRPM1) protein expression in mammalian retina.

\section{Methods}

One group of mice received unilateral intravitreal injections of $0.4 \mu 10.01 \mathrm{M}$ zinc solution and the control group received PBS. ERGs were recorded at different intervals after injection of zinc ions. Retinal TRPM1 protein was detected with western blotting. Immunofluorescence was performed to detect rod bipolar cells and photoreceptor ribbons. $50 \mu \mathrm{l}$ of $0.05 \mathrm{M}$ zinc solution was injected intravitreally in a rhesus monkey and ERGs were obtained at $2 \mathrm{~h}$ after injection.

\section{Results}

From 1 h to 8 weeks after zinc injections, mouse ERGs exhibited similar changes and a negative ERG waveform was evident: the dark- and light-adapted ERG b-wave decreased while the a-wave was relatively unchanged. Similar ERGs were also observed in monkey. The monkey ON-OFF responses showed attenuation of b-wave but relatively unaffected a-wave. TRPM1 protein expression was decreased in zinc-treated mouse retina. Retinal morphology was normal at day 1 after zinc injection. However at 8 weeks the inner nuclear layer became thinner. Aberrant dendritic processes of rod bipolar cells projected into the outer nuclear layer (ONL). The number of the photoreceptor synaptic ribbon decreased and ectopic ribbons were seen in the ONL.

\section{Conclusion}

Exogenous zinc ions interrupted retinal signal transmission from photoreceptors to ON bipolar cells but barely affected photoreceptor functions. Zinc downregulated TPPM1 expression implying TRPM1 may be a potential target of zinc mediated interruption instantly after injection. Abnormalities of inner retina structure may contribute to the long term defects of ON pathway dysfunction.

\section{OS2: Oral paper 3}

Cone spatial density and mfERG a-wave amplitude: a comparison across retinal eccentricity

\section{Viswanathan $\mathrm{S}^{1}$, Klein $\mathrm{MW}^{2}$, Burns $\mathrm{SA}^{2}$, Gang Huang ${ }^{3}$ \\ ${ }^{1}$ College of Optometry, State University of New York, USA, ${ }^{2}$ Kansas City Veterans Affairs Medical Center, USA, ${ }^{3}$ Indiana University School of Optometry, USA}

\section{Purpose}

Studies that have used pharmacological agents in non-human primates (e.g., Hood et al., IOVS 2002) indicate that electrical activity of cone photoreceptors, depolarizing cone bipolar cells and horizontal cells are all likely to contribute to mfERG a-wave. The purpose of this study was to examine the relationship between the mfERG a-wave and co-localized cone spatial density individually measured in young healthy human subjects. Methods

MfERGs (0.1-300 Hz) were recorded from 4 subjects (20-29 yrs) with a system from Veris Science (EDI, Inc.) using 2.4 degree unstretched hexagons from 206 retinal locations presented at 30 frames per m-step on a $75 \mathrm{~Hz}$ monitor with m-sequence exponent of 9 and flash strength $9.9 \mathrm{~cd} \mathrm{~s} / \mathrm{m}^{2}$. MfERG a-wave amplitude was measured from baseline at $10 \mathrm{~ms}$ on the leading edge of the a-wave. In vivo cone images were obtained at 24 retinal locations using a custom-built adaptive optics confocal scanning laser ophthalmoscope. Cone spatial 
density was measured from a $100 \times 100 \mu \mathrm{m}^{2}$ area centered on the mfERG hexagons at 24 retinal locations.

\section{Results}

MfERG a-wave amplitude as well as cone density reduced with increase in retinal eccentricity from the fovea and the a-wave amplitude and cone density were positively correlated for each subject $\left(\mathrm{r}^{2}=0.35\right.$ to 0.49 and $p=0.0049$ to 0.0002$)$. The coefficient of variation $(\mathrm{CV})$ of the mfERG a-wave amplitude across subjects at each retinal location (16-62\%) was larger than the $\mathrm{CV}$ of the cone density $(8-37 \%)$ at the same location. In addition, the a-wave amplitude calculated per cone remains roughly constant across eccentricity (approximately $11.5 \mathrm{pV}$ ). Conclusions

The results indicate that underlying cone density can account for a significant portion of the variance in the mfERG a-wave amplitude across retinal eccentricity but that additional factors are likely to make contributions to the variance of the measured mfERG parameters. The constant value of a-wave amplitude per cone across retinal eccentricity may result from changes in the relative contributions of cones and off cone bipolar cells and/or horizontal cells.

\section{OS2: Oral paper 4}

Contributions of human rod and cone pathways to different components of oscillatory potentials in the time and frequency domains

\section{Dai Jiaman, Shiying Li, Zheng Qin Yin, Gang Wang;} Xiaohong Meng

Department of Ophthalmology, Southwest Hospital, Southwest Eye Hospital, Third Military Medical University, Chongqing, China

\section{Purpose}

To investigate the contributions of human rod and cone pathways to different components of oscillatory potentials (OPs) in time and frequency domains.

\section{Methods}

Eighteen subjects were divided into three groups: normal group (NG), cone and rod pathway abnormal groups (CAG and RAG). All eyes were investigated by dark-adapted (DA) and light-adapted (LA) 3.0 ERG recommended by ISCEV. Data were output into the computer and processed and analyzed by the software Matlab 7.0.

\section{Results}

1. Compared with normal group, the amplitudes of the first two components of dark-adapted OPs in the cone dystrophy group were significant decreased. 2. Frequency domain analysis of LA and DA OPs in the three groups comprised two components (harmonic and subharmonic). Compared with normal group, harmonic components of the other two groups were dramatically decreased. The harmonic component in RAG is least in DA OPs and the harmonic component in CAG is least for LA OPs. 3. The harmonic component in CAG was highest for DA OPs in the three groups. There was no significant differences in the other two groups.

\section{Conclusions}

Cone and rod pathways both contributed to the DA and LA OPs, and the contribution of the rod pathway was greater than that of the cone pathway. Cone pathways mainly contributed to the earlier and faster components of OPs while the rod pathways mainly contributed to the later and slower components.

\section{OS2: Oral paper 5}

Multifocal pattern ERG: local differences in P50 vs $\mathbf{N 9 5}$
peak time; clue to origin?

\section{Bach M, Cuno AK, Hoffmann MB}

Ophthalmology Department, University of Freiburg, Germany

\section{Purpose}

The P50 component of the PERG arises mostly from the retinal ganglion cells (RGCs), but is not directly related to RGC spiking activity, in contrast to the N95 component. An open question is the local origin of the N95 component: does it originate from the RGC bodies or from the axon orientation change at the optic nerve head $(\mathrm{ONH})$ ?

\section{Methods}

Recording with PERG-optimized multifocal stimulation [1] in 21 subjects we obtained local PERGs for 36 retinal locations up to $20^{\circ}$ eccentricity and analyzed peak time topography of the grand mean traces.

\section{Results}

Peak times depended mostly on eccentricity: The macular P50 components had the highest peak times $(50 \mathrm{~ms})$, declining to $45 \mathrm{~ms}$ at $10^{\circ}$ and $43 \mathrm{~ms}$ at $20^{\circ}$ eccentricity. The subsequent negativity ("N95") had a similar overall topography, with highest peak times in the center $(76 \mathrm{~ms})$, declining to $66 \mathrm{~ms}$ at $20^{\circ}$. If the $\mathrm{N} 95$ were generated at the $\mathrm{ONH}$, the difference times of P50 and N95 $(\Delta \mathrm{t})$ should depend on axon length (path from RGC body to $\mathrm{ONH}$ ). There was indeed a local dependence of $\Delta \mathrm{t}$ on distance to $\mathrm{ONH}$ (up to $5 \mathrm{~ms}$ longer for temporal retina compared to nasal retina), but it was not fully described by retinal path length as calculated by line integrals based on Jansonius' [2] fiber tracking model.

\section{Conclusions}

(1) Macular RGCs are "slower". (2) N95-P50 peak time differences are roughly proportional to distance of $\mathrm{ONH}$. Temporal topography suggests (1) N95 origin is likely at the $\mathrm{ONH}$, and (2) axons traversing the macular region are slower.

[1] Hoffmann \& Flechner (2008), Clin Neurophys 119:409-417; [2] Jansonius et al. (2009) Vision Res 49:2157-2163.

\section{OS2: Oral paper 6}

The search for magno- and parvocellular contributions to the cortical visually evoked response

\section{Crewther D}

Centre for Human Psychopharmacology, Swinburne University of Technology, Hawthorn, Australia

\section{Purpose}

The search for cortical evoked responses identifiable as magnocellular $(\mathrm{M})$ or parvocellular $(\mathrm{P})$ in origin is complicated by the 
gold standard being single cell spike firing rates in primate LGN where the neurons are well separated anatomically. However, in human, the readily available techniques of electroencephalography, magnetoencephalography, and functional MRI measure different signals with different technology. Nonlinear Wiener kernel analysis visual evoked cortical potentials (VEP) offers some distinct advantages in isolating $\mathrm{M}$ and $\mathrm{P}$ generated waves. Such recordings offer diagnostic capability in schizophrenia, autism, dyslexia and dyscalculia, as well as in visual and retinal disease.

\section{Methods}

Nonlinear analysis of VEP (VERIS system) was recorded with 29 normal young adults with varying level of autistic tendency. Particular attention was paid to the responses from the foveal stimulus patch (subtending $5^{\circ}$ ), and the variation in responses with 5 levels of visual contrast $(10 \%, 25 \%, 50 \%, 70 \%$, $95 \%$ ). Magnetoencephalographic evoked fields (MEF) generated using a similar pseudorandom stimulus (contrasts $24 \%$, $95 \%$ ) developed using a VPixx/DataPixx combination were analysed across the 306 SQUID sensors on a population of 11 normal young adults.

\section{Results}

Contrast response functions were extracted from the major first and second order Wiener kernel peaks of the VEP. Naka-Rushton fits enabled contrast gain and semi-saturation contrasts to be elicited for each peak. A short latency second order response (M) with high contrast gain and a saturating contrast response function showed higher amplitude for the High autistic quotient (AQ) group (compared with Mid and Low groups) indicating poorer neural recovery after rapid stimulation. A longer latency non-linearity $(\mathrm{P})$ with no evidence of contrast saturation and lower contrast gain showed no difference between AQ groups across the full range of stimulus contrasts. MEG nonlinear analysis yielded similar responses, with the added source localization capability indicating that the earliest cortical responses $(\sim 50 \mathrm{~ms}$ ) occurred simultaneously over the occipital pole and an extrastriate location (MT/V5).

\section{Conclusions}

The cortical VEP first and second order responses can be decomposed into those with high contrast gain and contrast saturation (M-like) versus those with lower contrast gain and little evidence of saturation (P-like). Furthermore, the latency of the M-like components is approximately $25 \mathrm{~ms}$ less than those of the P-like components. Comparison of MEF with cortical VEP results gives strong confirmation of the $\mathrm{M}$ and $\mathrm{P}$ origins of the first and second slice major peaks respectively. In particular, the time/frequency MEG analysis differentiates the slices of the second order response, with the putative magnocellular response of the first slice showing much more gamma band activity, while the putative parvocellular response shows strong beta band activation but little gamma.

\section{Oral Session 3: Development and paediatrics}

\section{OS3: Oral paper 1}

Autofluorescence imaging in children with retinal pathology identified with visual electrophysiology
Chia A, Tan A, Boon Kwang L, Cheung G

Singapore National Eye Center, Singapore

\section{Purpose}

To determine if fundus autofluorescence (FAF) was useful in detecting retinal pathology identified with full-field or multifocal electroretinogram (ERG)

Methods

A retrospective study was performed of all children aged $<16$ years who had FAF imaging as well as abnormal ERG findings at the Singapore National Eye Center between 2007-2013. The FAF was reviewed by an ophthalmologist, masked to the ERG and clinical diagnosis, who rated the FAF to be normal or abnormal, and suggested a diagnosis.

Results

FAF of 76 eyes/39 children (mean age: $10.2 \pm 3.2$, range 4-16 yrs) with ERG/clinical diagnosis of cone/cone-rod dystrophy (CRD) (26 eyes), rod-cone dystrophy (RCD) (18), maculopathy (22), Stargardt disease (6), Best's macular dystrophy (BMD) (4) and congenital stationary night blindness (CSNB) (1) were reviewed. FAF could not be graded in 12 eyes ( $8 \%$ of CRD, $35 \% \mathrm{RCD}$, and $18 \%$ maculopathy). It was rated normal in 7 eyes ( $8 \%$ of CRD, $18 \% \mathrm{RCD}$ and $9 \%$ maculopathy), and abnormal in 57 eyes (84\% of CRD, $47 \%$ RCD, $73 \%$ maculopathy, and all Stargardt, BMD and CSNB eyes). Diagnosis based on FAF matched ERG in only $46 \%$. Decreased FAF at the fovea was associated with poorer VA (71 \% in eyes with VA $<6 / 36$ versus $40 \%$ with VA $>6 / 12$ ). Conclusion

FAF is a useful tool to assess children as it easier and less timeconsuming than visual electrophysiology. Qualitative grading of FAF images alone, however, was not always conclusive and electrophysiology was still necessary to confirm diagnosis in some cases.

\section{OS3: Oral paper 2}

Evidence of a delayed retinal-hole-like degeneration of the inferior retina in the juvenile model of lightinduced retinopathy (LIR)

Polosa A, Lachapelle P

Ophthalmology/Neurology-Neurosurgery, McGill

University/Montreal Children's Hospital, Montreal, Canada

\section{Purpose}

At the onset of juvenile light-induced retinopathy (LIR), retinal damage occurs mainly in a specific region of the superior retina; a retinal-hole-like area of depleted photoreceptors that continues to grow long after the cessation of light exposure (Polosa et al., ARVO 2011). The aim of this study was to document the retinotopic distribution and the long term consequences of light damage in the inferior retina, known to be more resistant, as well as to compare the kinetics of photoreceptor cell death in different regions of both hemiretinas.

\section{Methods}

Juvenile albino Sprague-Dawley rats $(n=65)$ were exposed to a bright cyclic light (10 klux) from P14 to 28. Structural (histology) and functional (mfERG) analyses were performed 
at selected postnatal days $(\mathrm{P})$ that were regrouped in 5 phases (p): p1 (P30), p2 (P35-50), p3 (P55-60), p4 (P90-120) and p5 $(\mathrm{P} 400)$. Four predetermined retinal regions $(\mathrm{R})$ were analyzed in both superior (S) and inferior (I) retina: [R1 $680 \mu \mathrm{m}$ from the optic nerve head, R2 680 to $1,700 \mu \mathrm{m}, \mathrm{R} 31700$ to $2,720 \mu \mathrm{m}$ and R4 3060 to $4080 \mu \mathrm{m}$; ora serrata].

\section{Results}

In controls, a gradual thinning of the outer nuclear layer (ONL) was noted as the rat aged, with no evidence of hemiretinal differences. In LIR rats, a uniform, but significantly greater than control thinning of the ONL occurred in the inferior retina during the first 3 phases [mean loss of $24 \pm 6 \%$ vs $13 \pm 3 \%$ in controls, $p<0.05]$. From $\mathrm{p} 4$, a thinner ONL was observed in IR2 and IR3 [35 $\pm 1 \%$ of control, $p<0.05]$ compared to the other retinal locations [IR1: $42 \%$ and IR4: $50 \%$ of control, $p>0.05$ ], giving rise to a similar retinal-hole-like area as previously reported for the superior retina. With further aging, a faster than normal rate of ONL loss occurred in all regions [mean loss of $90 \pm 7 \%$ vs $26 \pm 3 \%$ in control, $p<0.05$ ], resulting in an additional extent of this inferior retinal hole, a growth that also surpassed that of the retinal hole of the superior retina [SR3 (ONL loss of $64 \%$ ) and SR4 (ONL loss of $53 \%)$ ]. Hence, by P400, SR4 stood out as the most resistant retinal area [ONL: $32 \%$ of control, $p<0.05$ ]. These hemiretinal differences were also reflected in the mfERGs.

\section{Conclusions}

Our results thus demonstrate that despite being initially less affected, a pathophysiological process is nonetheless triggered in the inferior retina. Interestingly, our data also show that the emergence and the progression of the retinal-hole-like area is not a feature unique to the superior retina, but also occurs in the inferior retina, albeit with a temporal delay of 2 months compared to the first evidence observed in the superior retina. These findings thus suggest that the normal aging process cannot solely explain the rate of cell death in our LIR model and that another component, such as a secondary slow degenerative process initially triggered by light exposure, could be behind the hemiretinal difference noted in the rate of progression. Further research is however needed to better understand the exact mechanisms underlying this more chronic phase of LIR degeneration.

Funded by NSERC

\section{OS3: Oral paper 3}

Full-field flash ERG follow-up for children with a history of pre-threshold or threshold ROP

\section{Ping Wang, Tang Jin}

Ophthalmology Department, Hunan Children's Hospital, Changsha, China

\section{Purpose}

To evaluate the characteristics of the ERG in children with a history of pre-threshold or threshold ROP

\section{Methods}

A total of 24 children (48 eyes) with a history of pre-threshold ROP or threshold ROP received ERG examination. The rod response, maximal rod-cone response and cone response of full-field ERGs were recorded respectively following the
International Standard Protocol of ISCEV (2000 version). The latency and amplitude of a-wave and b-wave of various responses were analyzed. Ten children with a history of premature birth (20 eyes) were recruited as control group. This trial was approved by the Ethic Committee of Hunan Children's Hospital and informed consent was obtained from the parents of patients prior to any medical procedure.

Results

A-wave and b-wave of each response were observed. There were significant differences between the ROP and control groups for rod responses $(P<0.05)$; the latency was longer and the amplitude was lower in the ROP group. However, there was no difference between the ROP and control groups for cone responses $(P>0.05)$.

\section{Conclusion}

The retinal function of ROP children is abnormal: ERGs in ROP children with a history of pre-threshold or threshold ROP is abnormal compared to children with a history of prematurity but without ROP, mainly in the rod response. The full-field ERG is useful to evaluate retinal function in ROP children.

\section{OS3: Oral paper 4}

\section{Blindness following neonatal hypoxia-ischemia: retinal or cortical?}

Jung $S^{1}$, Polosa $A^{2,3}$, Lachapelle $P^{2,3}$, Wintermark $P^{1,3}$

${ }^{1}$ Department of Pediatrics, Division of Newborn Medicine, ${ }^{2}$ Department of Ophthalmology, ${ }^{3}$ Department of Neurology and Neurosurgery, Montreal Children's Hospital-Research Institute, McGill University, Montreal, Canada

\section{Purpose}

Neonatal hypoxia-ischemia (HI) is a leading cause of childhood blindness, which is thought to be due to brain injuries resulting from HI. However, it is probable that the immature retina would also be susceptible to $\mathrm{HI}$-induced injuries. The purpose of this study was to determine the effects of neonatal HI on the structure and function of the retina.

\section{Methods}

HI was induced in 10-day-old male Long-Evans rat pups by left common carotid artery ligation followed by a 2-hour exposure to $8 \%$ oxygen (Vannucci procedure). Sham operated pups served as control. Flash ERGs (fERGs) and VEPs (fVEPs) were obtained at postnatal days (P) 17, 22, 30 and 60 from the ipsi- and contralateral eyes. Eyes were subsequently collected for retinal histology (P60). In a separate cohort, mfERGs were obtained from both eyes at P90.

\section{Results}

HI animals were classified as mild $(n=6)$, moderate $(n=5)$ and severe $(n=6)$ subgroups based on the ERG b-wave amplitude of the ipsilateral (to carotid ligation) eyes compared to control. In control, the ERG showed an increase in amplitude from P17 through P22 (scotopic a- and photopic b-waves) or P30 (scotopic b-wave). The scotopic a-wave amplitude was not significantly different among groups at each age. The scotopic and photopic b-wave amplitudes were attenuated at P17 (scotopic: by $37 \%, 54 \%, 100 \%$; photopic: by $28 \%, 78 \%$, $99 \%$; mild, moderate, severe HI, respectively). Interestingly in 
the mild HI group, the b-wave amplitudes increased to normal values over time whereas in moderate- and severe HI groups, the increase was only marginal. In line with these findings, structural damage was observed primarily in the inner retinal layers. The extent of inner retinal destruction correlated with the severity of functional impairment. Furthermore, inner retinal damage was heterogeneous across the retina, which was also reflected in the mfERG. Contralateral eyes showed normal fERG, mfERG and histology. VEPs, however, showed abnormal findings when driven by either the ipsilateral or contralateral eyes of the HI animals.

\section{Conclusions}

We demonstrated that neonatal HI causes a retinopathy (of varying severity) that primarily affects the inner retina. Interestingly, there was evidence of functional recovery, especially in the mild HI group that assumed normal ERG function over time. This may be due to the varying extent of structural damage following an HI event. Moreover, histological and functional (mfERG) evidence suggest that there are regions of increased susceptibility within the retina. Abnormality in the VEP driven by the normal fellow eye is most probably due to cortical deficit. These findings suggest that visual impairments in asphyxiated newborns may arise from the retina, brain, or combination of both. Consequently, their retina and its function should also be monitored.

Funding: CIHR, FRSQ, SickKids Foundation, Réseau Vision, RI-MUHC/MCH

\section{OS3: Oral paper 5}

ERG diagnosis of Vigabatrin toxicity in infancy is predictive of oculo-visual defect in childhood

\section{Kumarappah A, Westall C}

The Hospital for Sick Children, University of Toronto, Canada

\section{Purpose}

Vigabatrin (VGB) is a drug used in the management of seizures. Visual field reductions are associated with VGB use in $30-50 \%$ of adults treated. In a pediatric population with the seizure type infantile spasms (IS), the light-adapted flicker ERG amplitude is the surrogate marker for VGB toxicity. Our prospective IS VGB cohort study (1999-2012), including 160 children with baseline (no VGB) ERG and at least 2 subsequent ERGs during VGB treatment, revealed that 33 $(21 \%)$ children developed VGB retinal defect (VGB-RD). VGB-RD is defined as significant reduction in age-corrected $2.29 \mathrm{~Hz}$ flicker ERG amplitude from baseline ERG on two consecutive visits. The purpose of the current study was to determine if VGB-RD in early childhood is correlated with an oculo-visual defect later in childhood.

\section{Methods}

Prospective cross-sectional oculo-visual assessment of visual fields (Goldmann kinetic perimetry) and retinal nerve fibre layer (RNFL) thickness (spectral domain OCT, Cirrus; Carl Zeiss Meditec) of children followed in the IS VGB cohort study. In the current oculo-visual cohort all participants were over 7 years of age at testing.

\section{Results}

22 children (age: 7-23 years) assessed previously in the IS VGB cohort study in infancy and early childhood participated; duration of VGB treatment 3 months -12 years. From the IS VGB cohort study in early childhood 8 of the children had demonstrated VGB-RD and 14 had no evidence of VGB-RD. 2/8 with VGB-RD had reliable visual field assessment and both had temporal visual field defect. Four with VGB-RD showed reliable OCT and all had reduced RNFL thickness. Of the 14 children who had not previously been identified with VGB-RD; 8 had reliable VF and OCT and all had visual fields and RNFL thickness within normal limits.

\section{Conclusions}

The RNFL was attenuated in all children who showed a reduction in the visual fields. $2.29 \mathrm{~Hz}$ flicker ERG amplitude is a predictor of reduction in visual field (temporal meridian) and reduction in RNFL thickness.

Funding: Lunbeck LLC, Canadian Institutes for Health Research, Vision Sciences Research Canadian Program Scholarship, University of Toronto Fellowship

\section{OS3: Oral paper 6}

Vision in six-month-old infants born to drug-misusing mothers prescribed methadone in pregnancy Hamilton $\mathbf{R}^{1}$, McGlone $\mathbf{L}^{2}$, McCulloch $\mathrm{DL}^{3}$,
MacKinnon $\mathbf{J}^{4}$, Bradnam $\mathbf{M S}^{1}$, Mactier $\mathbf{H}^{2}$

${ }^{1}$ Departments of Clinical Physics, Royal Hospital for Sick Children, Glasgow, and College of Medical, Veterinary and Life Sciences, University of Glasgow, Glasgow, UK,

${ }^{2}$ Neonatal Units, Princess Royal Maternity \& Royal Hospital for Sick Children, Glasgow, UK, ${ }^{3}$ Vision Sciences, Glasgow Caledonian University, Glasgow, UK,

${ }^{4}$ Department of Ophthalmology, NHS Highland, UK.

\section{Purpose}

Flash VEPs were abnormal in a cohort of 100 neonates exposed to maintenance methadone in utero. We now describe clinical visual and electrophysiological outcomes at six months.

\section{Methods}

Visual assessment included modified Atkinson test battery; strabismus, nystagmus, reduced visual acuity, delayed visual maturation or refractive error ( $>3$ dioptres) defined a failure. Pattern onset VEPs were recorded to $120^{\prime}, 60^{\prime}$ and $15^{\prime}$ checks.

\section{Results}

81 drug-exposed and 26 comparison infants (79 and $52 \%$ of the original cohorts) were assessed at a median age of 27 weeks (range 26-30). $90 \%$ of drug-exposed infants had been additionally exposed to illicit drugs and $26 \%$ to excess alcohol in utero. $40 \%$ of the drug-exposed cohort failed clinical visual assessment: the relative risk of abnormal assessment was 5.1 (95\% CI $1.3-20 ; p=0.02$ ). Nystagmus was particularly common. VEP peak times were slower and amplitudes smaller in drug-exposed infants, of whom $70 \%$ had one or more abnormal VEP parameter. Abnormal visual outcome at six months was not associated with the pattern of additional drug exposure nor with a history of neonatal abstinence. 


\section{Conclusions}

Abnormal visual electrophysiology in infants born to drugmisusing mothers prescribed maintenance methadone persists to six months of age, and is associated with abnormal clinical visual assessment.

Funding: Yorkhill Children's Charity

\section{Oral Session 4: Techniques}

\section{OS4: Oral paper 1}

Comparison of dark-adapted ERGs to standard and bright flashes in controls and patients

Hogg $C^{1}$, Shaheen $S^{1}$, Hamburg $S^{1}$, Lewis $S^{1}$, Singh $A^{1}$, Neveu MM ${ }^{1,2}$, Robson $A^{1,2}$, Holder $G^{1,2}$

${ }^{1}$ Electrophysiology Department, Moorfields Eye Hospital, London, UK, ${ }^{2}$ Institute of Ophthalmology, UCL, London, UK

\section{Purpose}

To compare the dark-adapted ISCEV-standard ERG (DA 3.0) with brighter flash ERGs (DA 10.0 and DA 30.0) in normal controls and clinical patients.

\section{Methods}

A retrospective analysis of full-field ERGs was performed in 78 consecutive patients. The diagnoses varied. Gold foil electrodes and the same Ganzfeld stimulator and recording system were used. All underwent full-field ERG testing according to the ISCEV standards. Dark-adapted ERGs were additionally recorded to bright flashes (DA 10.0 and DA 30.0) as suggested by ISCEV. The ERG a- wave data were compared with those obtained in 15 normal subjects.

\section{Results}

The DA 3.0 ERG a-wave frequently had a bifid like appearance in 54 patients and 7 controls. The first negative a-wave peak was more prominent than the second in 36 patients and in 4 controls; the second peak was more prominent or equal to the first in the remainder. The DA 10.0 and DA 30.0 ERG a-waves had only a single negative a-wave peak of higher amplitude than in the standard ERG in all cases. The DA 10.0 ERGs revealed subnormal a-waves in 3 of 78 cases with normal DA 3.0 ERGs (first negative peak). There was no significant difference between DA 30.0 ERG and DA 10.0 ERG amplitudes although the DA 30.0 ERG a-wave peak time was consistently shorter (mean peak time was $13 \mathrm{~ms}$ for DA 10.0 and $11 \mathrm{~ms}$ for DA 30.0). There was no significant difference in the mean amplitude or mean peak time of the b-waves between DA 10.0 and DA 30.0 ERGs, including those recorded from one patient with an electronegative ERG.

\section{Conclusion}

The ISCEV-standard DA 3.0 ERG a-wave shape is variable. The DA 10.0 ERG has a more consistent, larger and betterdefined a-wave facilitating measurement and reducing the chance of measurement errors. It can be more sensitive to rod photoreceptor dysfunction, reflecting the physiological origins of the components, as it has been previously established that only the first $8 \mathrm{~ms}$ or so of the a-wave have a photoreceptor origin. Increasing the flash strength from 10 to $30 \mathrm{~cd} \mathrm{~s} / \mathrm{m}^{2}$ provided no additional diagnostic information.

\section{OS4: Oral paper 2}

\section{Development of novel electroretinographic methods} to improve ERG recordings

Saul A, Still A

Department of Ophthalmology and Culver Vision

Discovery Institute, Georgia Regents University, USA

\section{Purpose}

Development of novel electroretinographic methods to permit: generalization of stimulation; relaxation of fixation requirements; artifact reduction; derivation of sensitive measures from kernels; and increased patient-friendliness.

\section{Methods}

A wide range of stimuli are presented to normal subjects. Fixation is toggled on and off, in order to compare fixation results to those in the presence of eye movements. Eye position is recorded using pupil tracking. For analysis, the stimulus is shifted at each time bin into the retinotopic coordinate system, with luminance averaged over each element in a grid. This retinotopic stimulus is then correlated with the signals from corneal electrodes, via wavelet correlation methods. This method performs temporal decorrelation. Spatial decorrelation is also required, and is complicated by the highly singular correlation matrices arising from the grids typically used.

\section{Results}

Interleaved trials with fixation on or off enabled direct comparisons between kernels derived in the absence or presence of eye movements. The two sets of kernels were correlated with each other significantly better than when retinal locations were scrambled. Artificial scotomas that moved on the stimulus monitor with eye movements could be recovered. The length of stimulus time required for convergence of kernels was $340 \pm 4 \mathrm{~s}$ vs $255 \pm 5 \mathrm{~s}$ for the fixation condition.

\section{OS4: Oral paper 3}

The extraction and analysis of $a-$ and $b$ - waves from human ERGs

\section{Zihe Chen, Chang-wei Zheng, Bo Lei}

Department of Ophthalmology, the First Affiliated Hospital of Chongqing Medical University and Chongqing Key Laboratory of Ophthalmology, Chongqing Eye Institute, Chongqing, China

\section{Purpose}

To determine the frequency range of the a-b wave complex in the dark- and light-adapted ERGs and to isolate the pure a- and b- waves. Methods

Full-field ERGs were recorded in 16 eyes of 8 normal volunteers. Digital filtering technique was used to extract the $a-$ and $b$-waves from dark- and light-adapted ERG. The timings of a- and b-waves were measured to determine the frequency range of $a-b$ wave complex. Major frequency components were determined from power spectra using fast Fourier transform (FFT). The effect of different order settings of the digital filter were compared to research the optimum condition where the oscillatory potentials 
(OPs) were completely removed while the amplitudes and phases of the a- and b- waves were less affected.

\section{Results}

The averaged frequency range of the dark-adapted a-b wave complex was between $14.99 \pm 2.39$ and $25.35 \pm 3.77 \mathrm{~Hz}$, compared with $25.22 \pm 6.56$ to $32.47 \pm 3.68 \mathrm{~Hz}$ for the lightadapted a-b wave complex, respectively, indicating the frequency range of the dark-adapted $a-b$ wave complex was significantly less than the light-adapted a-b wave complex $(p<0.001)$. A third order of the digital filter was the best choice in terms of removing the high frequency OPs from the waveform of the ERG and keeping the amplitude and phase of the a- and b- waves.

\section{Conclusions}

The frequency of a-b wave complex is lower than that of OPs. Therefore the a- and b- waves can be isolated from OPs using different digital filter settings for the human ERG. A third order filter and a bandpass of 1 to $45 \mathrm{~Hz}$ is the best choice to extract pure a- and b- waves from original ERGs.

\section{OS4: Oral paper 4}

Skewness of the distribution of normal ERG values and implications for calculation of the normal range Lee $\mathbf{M}^{1}$, Bach $M^{2}$, Sarossy $M^{1,3}$

${ }^{1}$ Faculty of Medicine, Nursing and Health Sciences, Monash University, Australia, ${ }^{2}$ Visual Function Section, University Eye Hospital, Freiburg, Germany, ${ }^{3}$ Centre for Eye Research Australia, Royal Victorian Eye and Ear Hospital, Melbourne, Australia

\section{Purpose}

ERG b-wave amplitudes from normal subjects are well known to have a positively skewed distribution. The normal distribution does not provide an adequate fit to the data and therefore should not be used to calculate $95 \%$ confidence intervals for the normal range. In this study, we determine the best fitting 2 parameter distributions for the data, analyze biases and explore the implications for normal range calculation.

\section{Methods}

66 eyes of 33 patients at 8 flash intensities $(0.01,0.033,0.1$, $0.33,1.0,3.3,10$ and $33 \mathrm{~cd} \mathrm{~s} / \mathrm{m}^{2}$ ) were included. Two parameter statistical distributions (normal, log normal, Weibull, gamma, inverse Gaussian and Gumbel) were fitted to the a- and b-wave amplitude data using R. Maximum likelihood was the fitting method used. The overall best method was determined by rank-sum of the Akaike information criterion (AIC) for each fit over the 8 intensity levels with the lowest rank-sum indicating the best fit. Confidence intervals were determined directly from the fitted distribution parameters. Simulation was used for each of the intensity levels to approximate the estimator bias for the upper and lower confidence intervals.

\section{Results}

For the a-wave amplitudes, the normal distribution was the best fit. For b-wave amplitudes the quality of fit by rank-sums from best to worst was Gumbel (13), log normal (24), gamma (27), inverse Gaussian (32), normal (33) and Weibull (39). For the intensity at $1 \mathrm{~cd} \mathrm{~s} / \mathrm{m}^{2}$ the mean was $343.6 \mu \mathrm{V}$ with the $95 \%$ confidence interval for the normal distribution 145.3 to $541.8 \mu \mathrm{V}$. Using the Gumbel distribution, the confidence interval was 203.1 to $570 \mu \mathrm{V}$. Simulation showed the $2.5 \%$ and $97.5 \%$ quantiles to be biased estimators. As the sample size increased from 20 to 200 the $2.5 \%$ confidence interval decreased from $220 \mu \mathrm{V}$ to an asymptote of $200 \mu \mathrm{V}$ and the $97.5 \%$ confidence interval increased from $520 \mu \mathrm{V}$ to an asymptote of $570 \mu \mathrm{V}$. A sample size of at least 50 is recommended based on these data.

\section{Conclusions}

This study confirms that the distribution of normal $b$ wave amplitudes is positively skewed. If a normal distribution is used to calculate the $95 \%$ confidence interval, the lower bound of the normal range will be significantly underestimated. We show an alternative method of calculating the confidence interval which allows for the skewness although bias needs to considered if the sample size is small.

\section{OS4: Oral paper 5}

Time-frequency domain analysis of the photopic hill reveals new and diagnostically relevant features of the human photopic ERG

\section{Gauvin $\mathbf{M}^{\mathbf{1}}$, Hébert $\mathbf{M}^{\mathbf{2}}$, Koenekoop $\mathbf{R K}^{\mathbf{1}}$, Little $\mathbf{J M}^{\mathbf{1}}$, Lina $\mathrm{JM}^{3,4}$, Lachapelle $\mathrm{P}^{1}$}

${ }^{1}$ Departments of Ophthalmology, Neurology and Neurosurgery, McGill University, Montreal Children's Hospital, ${ }^{2}$ Department of Ophthalmology, Centre de recherche Laval Robert-Giffard, Université Laval, ${ }^{3}$ Department of electrical engineering, École de Technologie Supérieure, ${ }^{4}$ Centre de Recherches Mathématiques, Montréal, Canada

\section{Purpose}

Analysis of clinical ERGs are usually limited to peak-time and amplitude (time-amplitude domain (TAD)) measurements of the a- and b-waves. However, it is well known that retinopathies can also markedly modify the morphology of the ERG signal, a feature potentially of diagnostic significance if it can be quantified in an objective, reliable and reproducible way. We examined if analysis of the ERG in the time-frequency domain (TFD), such as that offered with the discrete wavelet transform (DWT), could meet this challenge.

\section{Method}

Photopic ERGs obtained from normal subjects [ $\mathrm{n}=25 ; 21$ flash intensities: -2.23 to $2.84 \log \mathrm{cd} \mathrm{s} \mathrm{m}^{-2}$ (to obtain the photopic hill (PH)); background: $30 \mathrm{~cd} \mathrm{~m}^{-2}$ ] and patients presenting with a variety of retinal disorders $(n=10$; flash intensity: $0.64 \mathrm{log}$ cd s m${ }^{-2}$; background: $30 \mathrm{~cd} \mathrm{~m}^{-2}$ ) were analysed using novel DWT descriptors $(20 \mathrm{~Hz}$ a, $20 \mathrm{~Hz}$ b, $40 \mathrm{~Hz}$ a, $40 \mathrm{~Hz}$ b, $80 \mathrm{~Hz}$ OPs, $160 \mathrm{~Hz}$ OPs, $\Sigma$ Energy, $\Delta$ Variance and the Hölder exponent) and the resulting TFD PHs were compared [Pearson's correlation coefficient ( $\mathrm{r}$, ANOVAs] to the more traditional TAD PH (i.e. b-wave amplitude).

\section{Results}

The luminance-response functions generated using the TFD descriptors all presented with a photopic hill-like shape that was nearly identical to the classical $\mathrm{PH}$ obtained using b-wave 
amplitude measurements, with a match that was nearly perfect in some instances ( $\mathrm{r}$ values varying between 0.82 to $0.98 ; p<.001$ ), suggesting that the selected TFD descriptors appraised attributes of the cone ERG that co-varied with those obtained with TAD measures. Of interest, the variability (coefficient of variation $(\mathrm{CV})$ ) of DWT measurements was found to be significantly less compared to TAD measures of the same ERGs. For a $92 \%$ reduction in the signal to noise ratio (SNR) of ERGs, the CV of TAD measures increased by $>100 \%$ compared to $<50 \%$ for DWT measures. Finally, analysis of pathological ERGs showed that one (or more) TFD descriptor could be affected first (or significantly more) depending on the type of retinopathy and/or stage.

\section{Conclusion}

This study demonstrates, for the first time, that TFD analysis of the photopic ERG, such as that achieved with DWT derived descriptors, can be used to quantify the ERG beyond what can be accomplished using a TAD approach. Furthermore, our results also raise the possibility that a given retinopathy may impair more specifically one (or more than one) DWT descriptors, thus offering an alternative approach to identify (early diagnosis), classify and possibly stage the different pathophysiological processes that impair retinal function; an information that will be highly relevant especially in diagnostically challenging cases where the retinal disorder has not yet impaired TAD measures of the ERG.

Funded by the CIHR and the Réseau-Vision of the FRQS

\section{OS4: Oral paper 6}

\section{Scotopic dim blue and red ERG stimuli}

\section{Creel DJ}

Moran Eye Center, University of Utah School of Medicine, Salt Lake City, USA

\section{Purpose}

The ISCEV survey of ERG stimuli used other than the Standard protocol revealed few use balanced scotopic dim red and dim blue flash stimuli. Scotopic dim blue and red flashes are a useful addition to the Standard protocol.

\section{Methods}

The blue color should be less than $450 \mathrm{~nm}$, and the red above $585 \mathrm{~nm}$ to $700 \mathrm{~nm}$. These colors can be created with LED Ganzfeld stimulators or by placing color filters such as Wratten 47-47B for blue, and Wratten 26 for red over strobe lamps, and adjusting flash intensity so about a 200 microvolt b-wave is produced in a normal observer.

\section{Results}

The dim blue stimulus elicits a slow positive b-wave peaking at around $100-120 \mathrm{~ms}$. The dim red flash produces an ERG response composed of two parts: a fast b-wave peaking around $30 \mathrm{~ms}$ resembling a photopic single flash ERG, and a slow b-wave peaking about $100-120 \mathrm{~ms}$. The dim red flash ERG is a combination of cone and rod physiology with the conegenerated component occurring early, and a slower rod response similar to the b-wave produced when rod physiology is isolated using dim blue stimuli. The early component of the dim red ERG resembling a photopic single flash ERG is usually referred to as the $\mathrm{x}$-wave or bx wave.

\section{Conclusions}

In addition to more sensitive evaluation of rod physiology compared to the brighter dim white stimulus in the Standard protocol, cone function can be isolated allowing analysis of cone versus rod physiology in the dark-adapted eye. These scotopic blue and red dim flash stimuli can usually better detect retinal rod dysfunction, and the $\mathrm{x}$-wave complements predicting cone pathology along with photopic single flash and $30 \mathrm{~Hz}$ flicker ERGs. The scotopic dim blue and red flashes usually elicit the most sensitive ERGs in patients with early retinitis pigmentosa especially dominantly inherited forms, early stages of retinal toxicity, and most retinopathies.

\section{OS4: Oral paper 7}

The rise-time of the dark-adapted a-wave: a simple measure of rod sensitivity

\section{Robson J, Frishman L}

University of Houston College of Optometry, Houston, TX, USA

\section{Purpose}

To show how the timing of the peak and leading edge of darkadapted a-waves evoked by strong stimuli can be used to estimate rod sensitivity.

\section{Methods}

Computer simulations of the extracellular flow of photocurrent in the outer nuclear layer (ONL) and subretinal space of the dark-adapted retina have been used to predict the time course of the trans-retinal voltage generated by rods in response to brief ganzfeld flashes. The time course of simulated responses has been compared with that of ERG a-waves from primate and rodent retinas.

\section{Results}

Simulations show that the timecourse of the rod component of the trans-retinal voltage generated by a brief stimulus is dependent upon both the energy of the stimulus and the assumed sensitivity of the rods. When the energy of the stimulus is sufficiently increased, the simulated responses develop an initial negative nose as a result of a transient capacitive component of the photocurrent that flows in the ONL of the retina. ERG a-waves recorded in vivo from primate and rodent retinas that are evoked by strong stimuli reflect a similar transient nose. With stimuli strong enough to give a-waves with time-to-peak (implicit time) of less than about $12 \mathrm{~ms}$, the time for ERG a-waves to rise from $10 \%$ to $90 \%$ of their peak amplitude and the actual time of the peak after the stimulus both depend upon stimulus strength in just the way predicted by the simulations. Simulations indicate that the rise-time will be less affected than the time-to-peak by changes in stimulus duration, variations in the time delay in the rod transduction cascade or early postreceptoral contributions to the ERG.

\section{Conclusions}

The time for the ERG to rise from $10 \%$ to $90 \%$ of the amplitude of the a-wave (a quantity that is easily measured) can provide, for a stimulus of any given high energy, a useful simple measure of rod sensitivity.

Support:NEI (EY06671) 


\section{OS4: Oral paper 8}

\section{The subretinal implant alpha IMS to deliver useful vision in photoreceptor disease}

Zrenner $\mathbf{E}^{\mathbf{1}}$, Bartz-Schmidt KU ${ }^{\mathbf{1}}$, Gekeler $\mathbf{F}^{\mathbf{1}}$, Sachs $\mathbf{H}^{\mathbf{1}}$, Kusnyerik $A^{1,2}$ Stingl $K^{1}$ and SUBRET-Consortium

${ }^{1}$ Institute for Ophthalmic Research, Center for

Ophthalmology, University of Tuebingen;

${ }^{2}$ Department of Ophthalmology, Semmelweis-University,

Budapest, Hungary

\section{Purpose}

To restore useful visual sensations to patients blind from photoreceptor dystrophies by surgically implanting an externally powered photosensitive microphotodiode array with 1500 pixels in the subretinal space (1). A clinical trial has been performed to evaluate safety and efficacy concerning the visual function of patients who have undergone a unique surgical procedure to implant the chip (2).

\section{Methods}

A multicentre trial to implant and evaluate the photodiode chip is performed (www.clinicaltrials.gov, NCT01024803). Here we report on the first module of the trial and experiences with the implant surgery and function. Preoperatively, evaluation of visual function, extraocular movements, fundus photography, axial length measurements, OCT, FFA, electrophysiology, MRI of the orbit and psychological assessments were performed. A multidisciplinary team of surgeons performed the surgery. Post operative evaluation of the patient included electrophysiology, assessment of the chip function and assessment of the visual function with specialized computerized tests and activities of daily living.

\section{Results}

All the subjects had successful implantation of the chip. All subjects were able to perceive light after implantation of the photodiode chip. The visual experiences ranged from perception of light where there was none before surgery, to the ability to see individual letters $4 \mathrm{~cm}$ high at a working distance of $40 \mathrm{~cm}$. Motion detection was possible to angular speed up to $35 \mathrm{deg} / \mathrm{s}$, grating acuity up to 3.3 cycles per degree). In some cases visual acuity measurement with Landolt C-rings was possible up to Snellen visual acuity of 20/546. Additionally, the identification, localisation and discrimination of objects improved significantly in most patients. In repeated tests over a nine month period, several subjects were able to read letters spontaneously, controlled in four alternative forced choice tests (3). Control tests were performed each time with the implant's power source switched off.

\section{Conclusion}

The study has shown proof of concept that a photodiode chip placed in the subretinal space can provide useful vision for many subjects. Selection of patients based on experiences with preoperative OCT analysis, fluorescence angiography and autofluorescence helps to identify patients optimally suited for rehabilitation measures by means of subretinal electronic implants.

[1] Zrenner E, Nature Photonics 2012, 6: 344-345; [2] Zrenner E et al., Proc Biol Sci. 2011; 278:1489-97; [3] Stingl K et al. Proc. R. Soc. B 2013, 280, published online, 20 February 2013

\section{Oral Session 5: Optic nerve}

\section{OS5: Oral paper 1}

Evaluation of hemifield sector analysis protocol in mfVEP objective perimetry in the diagnosis and early detection of glaucomatous visual field defects

Ahmed Mousa M, Cubbidge RP, Al-Mansouri F, Bener A

Ophthalmology Investigative Unit, Ophthalmology

Department, Hamad Medical Corporation, Doha, Qatar

\section{Purpose}

The purpose of this study is to examine the effectiveness of a new analysis method of mfVEPs used for the objective assessment of the visual field in glaucoma patients, compared to the gold standard technique. Visual field assessment is a core component of glaucoma diagnosis and monitoring, and the standard automated perimetry (SAP) test is considered up until this moment the gold standard of visual field assessment. Although SAP is a subjective assessment and has many pitfalls, it is being constantly used in the diagnosis of visual field loss in glaucoma. MfVEP is a newly introduced method used for objective visual field assessment. Several analysis protocols have been tested to identify early visual field losses in glaucoma patients using the mfVEP technique: some were successful in detection of field defects, which were comparable to the standard SAP visual field assessment, and others were not very informative and needed more adjustment and research work. In this study, we implemented a novel analysis approach and evaluated its validity and whether it could be used effectively for early detection of visual field defects in glaucoma. Methods

Three groups were tested in this study; normal controls (38 eyes), glaucoma patients (36 eyes) and glaucoma suspect patients (38 eyes). All subjects had a two standard Humphrey visual field HFA test 24-2 and a single mfVEP test undertaken in one session. Analysis of the mfVEP results was done using the new analysis protocol; the hemifield sector analysis (HAS) protocol. Analysis of the HFA was done using the standard grading system.

\section{Results}

Analysis of mfVEP results showed that there was a statistically significant difference between the 3 groups of the mean signal to noise ratio (ANOVA $p<0.001$ with a $95 \% \mathrm{CI}$ ). The difference between superior and inferior hemispheres in all subjects were all statistically significant in the glaucoma patient group 11/11 sectors ( $t$ test $p<0.001$ ), partially significant $5 / 11$ (t-test $p<0.01)$ and no statistical difference between most sectors in normal group (only $1 / 11$ was significant) (t-test $p<0.9$ ). Sensitivity and specificity of the HSA protocol in detecting glaucoma were $97 \%$ and $86 \%$ respectively, while for glaucoma suspect were $89 \%$ and $79 \%$.

\section{Conclusion}

The results showed that the new analysis protocol was able to confirm already existing field defects detected by standard HFA, was able to differentiate between the 3 study groups with a clear distinction between normal and patients with suspected glaucoma; however the distinction between normal and glaucoma patients was especially clear and significant. The new HSA protocol used in mfVEP testing can be used to detect 
glaucomatous visual field defects in both glaucoma and glaucoma suspect patients. Use of this protocol can provide information about focal visual field differences across the horizontal midline, which can be utilized to differentiate between glaucoma and normal subjects. Sensitivity and specificity of the mfVEP test showed very promising results and correlated with other anatomical changes in glaucoma field loss.

\section{OS5: Oral paper 2}

Relationship among N2 amplitude of mfERGs, retinal sensitivity and ganglion cell complex in glaucomatous eyes

\section{Miura G, Shirato S, Kajita F, Yamamoto S}

Chiba University, Department of Ophthalmology and Visual Science, Chiba, Japan

\section{Purpose}

To investigate whether a significant correlation exists between the N2 amplitude of the mfERG, retinal sensitivity and ganglion cell complex (GCC) in glaucomatous eyes.

\section{Methods}

Twenty-four eyes of 24 glaucoma patients were studied. The mfERGs were elicited by red stimuli presented on a blue background. Responses from the central 5 elements within 20 degrees were analyzed. The N2 amplitudes were compared with the retinal sensitivity $(\mathrm{dB})$ measured by Humphrey field analyzer (HFA) and GCC by spectral-domain OCT of the corresponding area.

\section{Results}

There was a significant correlation between the $\mathrm{N} 2$ amplitude and the mean deviation values of HFA $(\mathrm{r}=0.51, P=0.02$ for superior retina; $\mathrm{r}=0.50, P=0.01$ for central retina; $\mathrm{r}=0.66$, $P=0.002$ for inferior retina). There was no significant correlation between the $\mathrm{N} 2$ amplitude and $\mathrm{GCC}(\mathrm{r}=0.37$ for superior retina, 0.35 for central retina and 0.17 for inferior retina).

\section{Conclusions}

The significant correlations between $\mathrm{N} 2$ amplitude and the mean deviation values of HFA suggest that the N2 amplitude of the mfERG receives some contribution from the retinal ganglion cells.

\section{OS5: Oral paper 3}

\section{Combined application of VEPs and mfERGs} in the diagnosis of optic nerve contusion

\section{Lei Zhang, Jing An, Qinglin Cao, Linsong Qi, Zuoming Zhang}

School of Aerospace Medicine, Fourth Military Medical University, Xi'an, China

\section{Purpose}

To investigate VEP and mfERG abnormality rates in the diagnosis of optic nerve contusion.

\section{Methods}

Unilateral contusion of 25 patients underwent visual electrophysiology testing, with the contralateral eye as control. Amplitude and latency of the VEP P100 and the response density of central mfERG was compared between the two groups. Results

VEP P100 amplitude was significantly lower; VEP P100 latency was significantly longer, and the response density of central mfERG was decreased in the contusion eye. The more severe the optic nerve conduction dysfunction, the more obvious mfERG abnormalities were.

\section{Conclusions}

The VEP is an objective assessment of optic nerve function. MfERG can reflect the multifocal function of retina. When the optic nerve is damaged, optic nerve fibers can stretch the macula which results in decreased mfERG responses. Thus, combined application of the VEP and the mfERG has great value in the diagnosis of traumatic optic neuropathy and improves early diagnosis.

\section{OS5: Oral paper 4}

\section{Functional and morphological analysis of glaucomatous retina by mfERG}

\section{Kajita F, Shirato S, Miura G, Yamamoto S}

Department of Ophthalmology and Visual Science, Chiba University Graduate School of Medicine, Chiba, Japan

\section{Purpose}

The aim of this study is to evaluate the relationship between electrophysiological function and micro-structure in glaucomatous retina by mfERG and spectral-domain OCT.

\section{Methods}

Twenty-four eyes of 24 glaucoma patients were studied. The mfERGs were elicited by red stimuli presented on a blue background at $9.4 \mathrm{~Hz}$. Responses from central 5 elements within 20 degrees were analyzed. The $\mathrm{N} 2$ amplitudes, measured at $80 \mathrm{~ms}$ from flash, were compared with the retinal nerve fiber layer thickness (RNFLT) by spectral-domain OCT (RT-vue100) of the corresponding area.

\section{Results}

There were significant correlations between $\mathrm{N} 2$ amplitude and RNFLT both for the superior and the central retina $(\mathrm{r}=0.58$, $P=0.005 ; \mathrm{r}=0.45, P=0.03$, respectively). However, there was no significant correlation in the inferior retina $(\mathrm{r}=0.29$, $P=0.17$ ).

\section{Conclusions}

The amplitude of 2 decreased as the RNFLT became smaller. The $\mathrm{N} 2$ amplitude reflects the function of the retinal ganglion cells.

\section{OS5: Oral paper 5}

The electrophysiological characteristics of ethambutol toxicity

Shiying $\mathrm{Li}^{1}$, Robson $\mathrm{AG}^{2,3}$, Neveu $\mathrm{MM}^{2,3}$, Zheng Qin Yin ${ }^{1}$, Holder GE ${ }^{2,3}$

${ }^{1}$ Southwest Hospital/Southwest Eye Hospital, Third Military Medical University, Chongqing, China, 
${ }^{2}$ Moorfields Eye Hospital, London, UK, ${ }^{3}$ UCL Institute of Ophthalmology, London, UK

\section{Purpose}

To investigate the electrophysiological and ophthalmic characteristics associated with ethambutol optic neuropathy (EON). Methods

Thirty patients with a diagnosis of EON underwent pattern reversal VEPs (PVEP), flash VEPs (FVEP), PERGs and full-field ERG examinations including 8 cases that were monitored over periods of up to 3 years. The clinical findings were reviewed retrospectively and compared with the electrophysiological findings.

\section{Results}

The majority of patients (28/30) with clinical evidence of EON showed delayed PVEP and FVEPs and 3 additionally showed an inter-hemispheric VEP timing asymmetry. The VEP abnormalities were greatest in those patients with severe vision loss. There was close correlation between the visual acuity (VA) drop of EON and the degree of peak-time delay in PVEP. Five of 25 subjects showed a reduced N95:P50 ratio in the PERG. Full-field ERGs were normal in all patients. Of the 8 subjects that were monitored, 6 showed improvement both in VA and VEP after ceasing ethambutol. There was no VA improvement in one patient with severely reduced N95:P50 ratios and in 1 other case that developed a vitreous haemorrhage in one eye.

\section{Conclusions}

Ethambutol toxicity may be associated with severe optic nerve and retinal ganglion cell dysfunction. Inter-hemispheric VEP asymmetry can occur and may imply chiasmal or retrochiasmal involvement. Severe PVEP abnormalities may resolve and the need for early diagnosis is highlighted.

\section{Oral Session 6: Animal studies}

\section{OS6: Oral paper 1}

Protective effect of anthocyanin extract from vaccinium uliginosum on rabbit retinaeagainst light-induced damage

\section{Lan Yin, Mao-nian Zhang}

Department of Ophthalmology, First Affiliated Hospital of the General Hospital of PLA, Beijing, China

\section{Purpose}

To study the effect of vaccinium uliginosum (VU) on the ERG and retinal pathological changes in rabbits after light-induced damage.

\section{Methods}

Twenty-eight Chinchilla rabbits were randomly divided into four groups: administration beforehand (A), administration after injury (B), light injury without administration (C), and blank group (D). After 4-week administration of VU homogenate once a day to group A, ERGs were recorded. Except for group D, the groups were exposed to strong light. Just after that, group A stopped receiving VU treatment but group B started. Then ERG in all groups was recorded after 1 day, 1 week, and 2 weeks. Finally, the tissues and structures of all the groups were observed and the thickness of the outer nuclear layers (ONL) was measured.

\section{Results}

(1) After four weeks of feeding with VU, latency of ERGs in group A became shorter than other groups and the amplitude increased. After being exposed to strong light, latency lengthened and amplitude decreased in all injured groups, but comparing at each time point, the measured values in group A were better than group C. With the accumulation of VU, ERGs in group B improved. Finally, all detected values became better than group C. (2) Retinae in group D were normal in histology and the layers were in order, but group $\mathrm{C}$ became disarranged. Injuries in group $\mathrm{A}$ and $\mathrm{B}$ were minor compared to $\mathrm{C}$. Thickness of ONL in group $\mathrm{C}$ was significantly thinner than other groups, and that in group A and B was thicker than C, while thinner than D. That in group A was thicker than B.

\section{Conclusions}

(1) VU can relieve injury to rabbit retinae exposed to normal day and night rhythm. (2) VU can relieve the harm caused by light when used beforehand. (3) VU can repair the light damage to retina.

\section{OS6: Oral paper 2}

\section{A rodent ERG system based on Maxwellian view optics} with green and UV light sources

\section{Massie N, Jungtae Rha}

Phoenix Research Laboratories, San Ramon, CA, USA

\section{Purpose}

To study the use of a ganzfeld ERG based on Maxwellian view optics with green and UV light sources as designed for rodents. Methods

A Maxwellian view ERG system was constructed with the ability to set levels, pulse lengths, timings, flicker and background in both green and UV wavelengths. This unit was tested with both mice and rats. One objective was to measure separately the responses of $\mathrm{S}$ and $\mathrm{M}$ cones, all with the Maxwellian view illumination rather than the classical Ganzfeld bowl.

Results

The Maxwellian view system, with the advantages of using single LEDs, was shown to provide an effective means of whole-eye ERG measurement. Mixing the green UV signals yielded additional insights into the $\mathrm{S}$ vs the $\mathrm{M}$ cone responses. Conclusions

This approach may prove more productive as the flexibility of the Maxwellian illumination with LEDs immediately gives access to various timings of pulses and $\mathrm{M}$ and $\mathrm{S}$ cones.

Financial declaration: N Massie is company owner of Phoenix Research Laboratories

\section{OS6: Oral paper 3}

Experimental research of light-induced retinal degeneration in Bama miniature pigs

\section{Jun Liu}

Key Lab of Visual Damage and Regeneration and Restoration of Chongqing, Southwest Hospital, Third Military Medical University, Chongqing, China 


\section{Purpose}

To explore the visible light continuously uninterrupted lighting on the retinal degeneration in Guangxi Bama miniature pigs.

\section{Methods}

Bama miniature pigs aged 3-4 months were exposed to continuous intermittent white light to induce slowly retinal degeneration. Fundus photography, OCT, full-field ERGs and mfERGs were utilized to compare the differences between normal pigs and pigs with light-induced retinal degeneration, identifying optical retinal degeneration model from form and function in vivo.

\section{Results}

Compared with normal pigs, no significant visible fundus photography changes were seen in the light-induced retinal degeneration pigs; nevertheless, the inner limiting membrane-retinal pigment epithelium layer was thinner on OCT (statistical significance at $P<0.05$ ), the amplitudes of dark-adapted combined rod-cone ERGs a-waves, b-waves and OPs, and light-adapted b-waves were decreased, p1 waves of the mfERG were also clearly decreased $(P<0.05)$ and the average amplitude density of $\mathrm{p} 1$ waves of the mfERG were significantly lower $(P<0.05)$.

\section{Conclusion}

Continuous, uninterrupted visible light can lead to modifications of structure and function in porcine retina: this may be an appropriate chronic retinal degeneration model.

\section{OS6: Oral paper 4}

ERG responses of Nrl knockout mice reveal two distinct signal pathways

\section{Haohua Qian, Yichao Li, Roger JE, Rachel RA, Swaroop A}

Visual Function Core, National Eye Institute, Bethesda, MD, USA

\section{Purpose}

$\mathrm{Nrl}$ is a transcription factor that controls the fate of photoreceptor progenitor cells. Lack of $\mathrm{Nrl}$ expression in Nrl-ko mouse retina leads to the conversion of rod photoreceptors to cone photoreceptors. The purpose of this study is to understand the changes in visual signal pathway and processing mechanisms in the cone-only retina of Nrl-ko mouse.

\section{Methods}

Adult wild type (WT) (C57BL/6J) and Nrl-ko animals (2-4 month old) were used in this study. ERG responses were recorded with Espion E2 system (Diagnosys LLC) equipped with UV $(365 \mathrm{~nm})$ and green $(514 \mathrm{~nm})$ LED. Expression patterns of M-opsin in the retina were examined by immunohistochemistry. The OptoMotry system (CerebralMechanics Inc.) was used to measure spatial sensitivity of mice by their opto-kinetic tracking (OKT) responses.

\section{Results}

Compared with WT animal, Nrl-ko mice exhibited larger b-wave amplitudes and a higher sensitivity for UV flash ERG $\left(\mathrm{R}_{\max }=550 \pm 35 \mu \mathrm{V}\right.$ and $273 \pm 25 \mu \mathrm{V}, \sigma=1.3 \times 10^{3}$ and $7.2 \times 10^{3} \varphi / \mu \mathrm{m}^{2}$ for Nrl-ko $(\mathrm{n}=6)$ and WT $(\mathrm{n}=4)$, respectively). On the other hand, Nrl-ko mice had smaller b-wave amplitudes and a lower sensitivity for ERG responses elicited by green light $\left(\mathrm{R}_{\max }=185 \pm 20 \mu \mathrm{V}\right.$ and $275 \pm 30 \mu \mathrm{V}$, $\sigma=1.0 \times 10^{5}$ and $2.3 \times 10^{4} \varphi / \mu \mathrm{m}^{2}$ for Nrl-ko $(\mathrm{n}=6)$ and WT $(n=4)$, respectively). ERG responses to green flashes had a faster kinetics with shortening in both latency and peak implicit time than those evoked by UV flashes $(n=6)$. UV flicker ERG responses had a frequency-response turning curve that lay in between WT scotopic and photopic response curves, whereas green flicker responses followed closely to WT photopic response curve. Spatial sensitivities measured by opto-kinetic tracking responses to visible light stimuli were $0.354 \pm 0.001 \mathrm{c} / \mathrm{deg}$ for Nrl-ko $(\mathrm{n}=4)$ and $0.384 \pm 0.002 \mathrm{c} / \mathrm{deg}$ for WT $(\mathrm{n}=5)$. Immunohistochemistry revealed $\mathrm{M}$-opsin was preferentially expressed in a small subset of cone photoreceptors in Nrl-ko retina. Conclusions

Two subtypes of cone photoreceptors are present in the Nrl-ko animals. The majority of them express high levels of S-opsin and also communicate with "rod"-bipolar cells in the retina. This pathway mediates large, highly sensitive, and slow UV flash ERG responses. M-opsin is preferentially expressed in a small subset of cone photoreceptors, and these photoreceptors likely only connect with cone-bipolar cells. Spatial sensitivity determined by visible-wavelength light is largely mediated by this pathway.

\section{OS6: Oral paper 5}

Inhibition of IGF-IR alters the in vitro neural differentiation of rat optic cup-derived retinal stem cells

Xiaoyong Huang, Zheng Qin Yin, Qiyou Li, Haiwei Xu, Yuxiao Zeng

Southwest Hospital/Southwest Eye Hospital, Third Military Medical University, Chongqing, China

\section{Purpose}

IGF-1R was evaluated to promote the differentiation rate of optic cup-derived retinal stem cells (OC-RSCs) to photoreceptors.

\section{Methods}

Immunohistochemistry, immunocytochemistry, Western-blot, and flow cytometry.

\section{Results}

$\sim 90 \%$ of differentiated OC-RSCs expressed the Thy 1.1 antigen and the proportion of Thy 1.1 cells appeared to be FBS concentration dependent. Thy $1.1+$ cells decrease significantly by treatment of IGF-1Ra antibody, and the same changes is observed in $\mathrm{PKC}+$ cells production. However, rhodopsin + cells increased significantly under the treatment of IGF-1Ra antibody. Conclusion

OC-RSC is a feasible cell model in studying RGCs development. These results suggest that IGF-IR inhibition can remodel the differentiation bias of OC-RSCs towards photoreceptor lineages and plays a key role in RGC differentiation.

\section{OS6: Oral paper 6}

Long-term preservation of cone vision following rodspecific gene therapy in experimental retinitis pigmentosa 
Seeliger $\mathbf{M W}^{1}$, Sothilingam $\mathbf{V}^{1}$, Koch $\mathrm{S}^{2}$, Tanimoto $\mathbf{N}^{1}$, Garcia Garrido $\mathbf{M}^{1}$, Biel $\mathbf{M}^{\mathbf{2}}$, Mühlfriedel $\mathbf{R}^{1}$, Michalakis $\mathbf{S}^{2}$

${ }^{1}$ Division of Ocular Neurodegeneration, Institute for Ophthalmic Research, Centre for Ophthalmology, Eberhard Karls-Universität, Tuebingen, Germany, ${ }^{2}$ Center for Integrated Protein Science Munich (CIPSM), Department of Pharmacy-Center for Drug Research, Ludwig-

Maximilians-Universität, Munich, Germany

\section{Purpose}

Retinitis pigmentosa (RP) is a severe and frequently blinding form of human retinal degeneration. The term denotes a genetically and phenotypically heterogeneous group of diseases in which the loss of the primarily affected rod photoreceptors is followed by secondary dysfunction and decay of cones ('bystander effect'). Here, we assess the effect of rod-specific gene therapy in experimental RP on long-term preservation of cone vision.

\section{Methods}

Mice lacking the $\beta$ subunit of the rod cyclic nucelotide gated (CNG) channel (Cngb1-/-), a deficiency that leads to RP in human patients, were treated by adeno-associated virus (AAV)mediated gene replacement therapy. Specifically, rAAVs expressing mouse CNGB1 under control of a rhodopsin promoter were injected into the subretinal space of 2 weekold knockout mice. The treatment success was monitored longitudinally in vivo for 9 to 18 months using ganzfeld ERGs, scanning laser ophthalmoscopy (SLO), OCT, and was compared to immunohistochemical ex vivo data.

\section{Results}

Based on the degeneration of cones secondary to rods in RP, it is a common hypothesis that, conversely, preservation of rods might save cones from degeneration, but this has to our knowledge not yet been demonstrated. Our long-term functional and imaging data now show that in fact it is possible to preserve cone survival in the area of treatment. We found that in the rescue area, extending to about $1 / 3$ to $1 / 4$ of the retina, a substantial portion of rods were still present at the end of the observation period. In this region, cones were also morphologically present, and signs of regular outer retinal layering was found on OCT, including the OS/IS border. Functionally, there was no difference between cone ERGs in treated and untreated eyes at early post-injectional time points, but a marked difference at the late time points, when the outer retina had practically vanished in the untreated regions.

\section{Conclusions}

In this work, we present long-term data for rod and cone survival and functionality following gene therapy in a model of RP with a targeted deletion of the $\beta$ subunit of rod CNG channels (Cngb1-/-). Our results provide clear evidence that the maintenance of morphological and functional integrity of the cone system is topographically limited to areas of rod rescue. These findings settle a longstanding issue regarding the importance of the rod system for cone survival, and are a major advance towards strategies for the therapeutic rescue and the preservation of visual function in RP.
Oral Session 7: Electrophysiology across the visual pathway

\section{OS7: Oral paper 1}

\section{Effects of visual acuity of cataract patients on the mfERG}

Jing An, Zuoming Zhang, Lei Zhang

School of Aerospace Medicine, Fourth Military Medical University, Xi'an, China

\section{Purpose}

To evaluate the correlation between visual acuity (VA) and the mfERG in cataract patients.

\section{Methods}

The amplitude of the ERG was used to choose cataract patients who had no significant eye disease except for cataract. VA before and after cataract surgery, VEPs, ERGs and mfERGs were measured from 140 subjects with cataract and 20 subjects with normal ocular health as the normal controls. The subjects were divided into eight groups according to their degree of visual acuity. $95 \%$ confidence intervals (CI) were calculated for different VA.

\section{Results}

VEP amplitudes were significantly reduced in cataract patients whose VA was less than 0.3. MfERG amplitudes of the central retina $\left(5^{\circ}\right)$ were significantly reduced when VA less than 0.7 compared with that of control groups. Compared with VEP amplitude, central point mfERG amplitude was much more closely related to VA before surgery. For VA of hand movement, $95 \%$ CI for mean amplitude of centre point mfERG was 4.71-8.28. For VA of counting fingers, $95 \%$ CI was 3.51-9.53. For VA from 0.01 to $0.1,95 \%$ CI was 8.91-10.97. For VA from 0.1 to $0.2,95 \%$ CI was 11.86-14.19. For VA from 0.2 to 0.3 , $95 \% \mathrm{CI}$ was $12.46-14.18$. For VA from 0.3 to $0.5,95 \% \mathrm{CI}$ was 13.31-16.22. For VA from 0.5 to $0.7,95 \%$ CI was $14.94-18.00$. For VA more than 0.7, $95 \%$ CI was 19.23-23.23. The mfERG paracentral amplitude and VA after surgery had little correlation. The VA of post cataract surgery most of them were more than 0.5 for the ratio was $95.9 \%$.

\section{Conclusions}

The correlation of VA in cataract patients with the amplitude of central point mfERG was closer than with VEP. The $95 \% \mathrm{CI}$ of central point mfERG were given in different visual acuities in simple cataract, and could help to evaluate macular function and guide clinical prognostic evaluation of patients with cataract. The central point mfERG was one of the best items to evaluate VA after surgery.

\section{OS7: Oral paper 2}

The mfERG and OCT data associations in macular hole surgery

Zueva M, Neroev V, Tsapenko I, Bychkov P, Sarygina O, Ilyukhin P, Semenova $\mathbf{N}$

Moscow Helmholtz Research Institute of Eye Disesases, Moscow, Russian Federation 


\section{Purpose}

To determine mfERG and OCT parameter correlations after the surgical closure of idiopathic macular full-thickness holes $(\mathrm{MH})$. Methods

12 patients with $\mathrm{MH}$ (3-4 stage by Gass) were tested before and 1, 3 and 6 months after subtotal vitrectomy with inner limiting membrane peeling. The $\mathrm{MH}$ duration was $6-8$ months. Stratus 3000 OCT was used to detect the maximal basal and minimal apical size of MH, total macular volume (TMV), retinal thickness (RT) within $1 \mathrm{~mm}$ (foveal thickness FT), at the inner (1-3 mm) and outer (3-6 mm) scanning diameters to the MH center. MfERG (61 hexagons) was recorded with RETIport/scan21 (Roland Consult); P1 and N1 were analyzed by rings (R1-R5).

\section{Results}

Before surgery, best corrected visual acuity (BCVA) was $0.2 \pm 0.06$. The basal MH diameter was $612 \pm 91 \mu \mathrm{m}$ and the apical diameter $312 \pm 25 \mu \mathrm{m}$. The average FT was $338 \pm 18$ $\mu \mathrm{m}$ and RT at the outer scanning diameter was $306 \pm 8 \mu \mathrm{m}$, and $237 \pm 7 \mu \mathrm{m}$ at the inner diameter. TMV was $7.25 \pm 0.1 \mathrm{~mm}^{3}$. One month after surgery, BCVA increased up to $0.46 \pm 0.06$, $p<0.05$. In rings $\mathrm{R} 2$ and $\mathrm{R} 3$, the mfERG $\mathrm{P} 1$ was reduced less sharply than in R1, but did not improve for a longer period. BCVA before the surgery correlated with P1 density in R1. In the postsurgery period, high associations were found between the BCVA, basal MH diameter and P1 density ratio in R1/R2 $(p=0.0017$, $\mathrm{r}=0.8)$ and $\mathrm{R} 1 / \mathrm{R} 3(p=0.02, \mathrm{r}=0.65)$. For apical MH diameter, a correlation with $\mathrm{P} 1$ latency in $\mathrm{R} 4(p=0.028, \mathrm{r}=-0.656)$ and $\mathrm{R} 5$ rings ( $p=0.016, \mathrm{r}=-0.7)$ was detected. BCVA and $\mathrm{RT}$ were intimately related to the mfERG N1 latency in R2. After one and three months, a direct correlation was observed between average N1 latency and the photopic flicker ERG at 10 and $12 \mathrm{~Hz}$.

\section{Conclusions}

Strict correlations were detected between the OCT, BCVA, and mfERG data. The mfERG changes suggest that bigger basal MH size influences the retina function in the fovea and parafoveal zones, possibly determining the post-surgical functional outcome. N1 latency tested in the R2 ring may be a good indicator of central cone functional recovery.

\section{OS7: Oral paper 3}

\section{Comparison of cone ERGs after indocyanine green-, brilliant blue G-, or triamcinolone acetonide-assisted macular hole surgery}

\section{Shigeki M, Toba Y, Nishimura T, Ohzeki T, Murai K, Kurosaka D}

Department of Ophthalmology, Iwate Medical University School of Medicine, Iwate, Japan

\section{Purpose}

The photopic negative response (PhNR) of the cone ERG originates from the neural activity of retinal ganglion cells (RGCs). Indocyanine green (ICG) and other dyes are used during macular hole $(\mathrm{MH})$ surgery to make the internal limiting membrane (ILM) more visible. However, ICG has been shown to be toxic on RGCs in animal studies, and Ueno et al. demonstrated that the PhNR amplitude was selectively reduced in patients treated with macular hole surgery using ICG (IOVS,
2006). We compared the RGC function using the PhNR in patients who underwent ICG-, brilliant blue G- (BBG-), and triamcinolone acetonide (TA)-assisted ILM peeling during MH surgery.

\section{Method}

Forty-eight eyes of 48 patients with a macular hole were randomly divided into those undergoing ICG-, BBG-, or TA-assisted vitrectomy ( $\mathrm{n}=16$ for each group). Their ages ranged from 47 to 76 with a mean of 65 years of age. All patients underwent cataract and macular hole surgery combined with ILM peeling. Full-field cone ERGs were recorded pre- and 1, 3, and 6 months postoperatively. The ERGs were elicited by red stimuli on a blue background. The amplitudes and implicit times of the a- and b-waves and the amplitudes of the OPs and PhNRs were measured. The mean deviations of standard automated perimetry (SAP) were used for the evaluations. The best-corrected visual acuity (BCVA) was measured at every visit. The circumferential retinal nerve fiber layer thickness (RNFLT) was measured by spectral domain OCT pre- and 6-months postoperatively.

\section{Results}

All macular holes were closed with a significant improvement of the BCVA and SAP, and there were no significant differences among the groups. There was no significant difference between the pre- and postoperative RNFLT in any group. The amplitudes of the a- and b-waves were not significantly changed postoperatively. The implicit time of these waves were significantly prolonged, and the summed OPs:b-wave amplitude ratio was significantly decreased postoperatively in all groups. These ERG changes were not significantly different among the groups. The postoperative PhNR:b-wave amplitude ratio was significantly lower in the ICG group than the BBG or TA group. This amplitude ratio was significantly reduced in the ICG and BBG groups at 1 month postoperatively compared to the preoperative value, while it remained unchanged in the TA group.

\section{Conclusions}

Although there were no significant differences in the BCVA and SAP among the groups, the PhNR was decreased in the ICG group. This indicates that the PhNR may detect subclinical impairments of the RGC caused by the toxic effect of ICG. We conclude that BBG and TA are safer than ICG for use during MH surgery.

\section{OS7: Oral paper 4}

\section{Clinical study of topical isopropyl unoprostone for retinitis pigmentosa}

\section{Yosuke N, Hagiwara A, Kumagai K, Yamamoto S}

Department of Ophthalmology and Visual Science, Chiba University Graduate School of Medicine, Chiba, Japan

\section{Purpose}

Recently we have reported results of the phase 2 clinical trial of topical $0.15 \%$ isopropyl unoprostone (IU), a BK-channel activator, for retinitis pigmentosa (RP). After 24 weeks of IU instillation, there was a significant dose-dependent responsiveness of the central $2^{\circ}$ retinal sensitivity (Ophthalmol Ther 2012;1:5). We reviewed the changes 2-year after the end of the IU treatment. 


\section{Methods}

We studied 22 eyes of $22 \mathrm{RP}$ patients who were enrolled in the P2 clinical trial (P2) for 24 weeks and followed thereafter. These patients had been randomly assigned to one of the three doublemasked treatments; high dose $(\mathrm{H}$, two drops/dose; $\mathrm{n}=6)$, low dose ( $\mathrm{L}$, one drop/dose; $\mathrm{n}=8)$, and placebo $(\mathrm{P}, \mathrm{n}=8)$. The therapy was terminated in all the patients after the 24 week trial. We examined the mean retinal sensitivity of four central sites using the Humphrey field analyzer (HFA) 10-2 program and best corrected visual acuity (BCVA), at the start and end of the clinical trial, and at 120 weeks after the start of the trial.

\section{Results}

At the end of the P2 trial, the $\mathrm{H}$ group showed a statistically significant improvement in central retinal sensitivity $(P=0.018)$. At 120 weeks after the start of the trial, the mean change in sensitivity of the four central sites were $1.52 \pm 2.33 \mathrm{~dB}$ for the $\mathrm{H}$ group, $-1.30 \pm 2.08 \mathrm{~dB}$ for the $\mathrm{L}$ group, and $-0.78 \pm 1.74 \mathrm{~dB}$ for the P group. Although differences among the three groups were not statistically significant, the degree of worsening was the least in the $\mathrm{H}$ group suggesting that the disease progression was slower than that in the L group after the ending of the therapy. No significant changes were observed in the final BCVA.

\section{Conclusions}

The retinal sensitivity in the $\mathrm{H}$ group was maintained even 2 years after the IU therapy was stopped. This suggests a longterm preservation of visual sensitivity by IU. Based on the results of the P2 trial, the P3 trial has just started in Japan. Onehundred-eighty RP patients will be randomly assigned to one of the two double-masked treatments; IU (two drops/dose) and placebo. The primary endpoint is changes of the mean retinal sensitivity of four central sites by HFA after 52 weeks.

\section{OS 7: Oral paper 5}

\section{Functional damage of eight patients with Leber's hereditary optic neuropathy}

\section{Wang M, Shiying Li, Gang Wang, Xiaohong Meng, Yi} Wang, Zhengqin Yin

Southwest Hospital/Southwest Eye Hospital, Third Military Medical University, Chongqing, China

\section{Purpose}

To observe the characteristics of functional damage in eight cases of Leber's hereditary optic neuropathy (LHON).

\section{Methods}

Eight patients with a mean age of 29 years (range 15-59 years) were diagnosed with LHON by mitochondrial DNA tests. We compared the functional tests of the subjects to normal values. Results

Five patients had visual field (VF) testing which demonstrated visual defect of the superior quadrant in association with enlarged blind spots. Five patients had flash VEPs: implicit times of P2 waves were all within the normal range. Four patients had pattern VEPs; three showed mildly prolonged P100 implicit time, one had moderately prolonged P100 implicit time. Six patients had PERG testing and five of them showed reduced value of N95/P50 in both eyes while the P50 amplitudes were normal. Seven patients had flash ERGs and the results were all normal. MfERGs of three patients manifested a decrease of amplitude density within $10^{\circ}$ of the macular area while higher amplitudes were found between $10^{\circ}$ to $30^{\circ}$, which is a very interesting and worth studying thing. Two patients had reduced amplitude density of the whole $30^{\circ}$ of macular area. Five patients' OCTs showed decreased ganglion cell and IPL complex layer in macular and retinal nerve fiber layer (RNFL) around optic disc.

\section{Conclusions}

From these patients, the decrease of N95/P50 value in the PERGs showed the damage of ganglion cells in LHON, which was confirmed by the structural loss in macular and RNFL shown on OCT. Retinal function of macula area was usually impaired. The latency of the VEP usually did not delay as much as seen in patients with retrobulbar optic neuritis.

\section{OS7: Oral paper 6}

Atypical features at the fovea on spectral domain OCT in cases with uncorrectable high hypermetropia

\section{Shehab A, Rady H}

Opthalmology Department, Minya University; Orman, Giza, Egypt

\section{Purpose}

To report atypical features at the fovea on spectral domain OCT in cases with uncorrectable high hypermetropia

Methods

4 cases ( 1 female at 6y, 3 males at 20, 35, 50 years of age) suffering from defective central vision in both eyes. BCVA ranges from 0.2 to 0.4 . Refraction ranges from +5 to +10 . Patients had complete ophthalmic examination. Patients were tested with electrophysiology (FERG, PERG, mfERG, PVEP), OCT and FA. Results

Fundus examination was normal except for flat foveal reflex OU. Normal FERG, PERG was abnormal, mfERG showed abnormal foveal peak, PVEP was normal or slightly delayed. FA showed absent or nearly absent FAZ. OCT showed in one case a grade 1 foveal hypoplasia while the other 3 cases showed loss of the foveal pit and localised loss of the ILM and abnormality of the inner retina with preservation of IS/OS integrity.

\section{Conclusions}

Correlation between electrophysiology, FA and OCT can confirm the diagnosis of isolated foveal hypoplasia in cases with uncorrectable high hypermetropia.

\section{OS7: Oral paper 7}

Waveform characteristics of optimized four-channel mfVEP with normal gaze and experimental scotoma in Chinese young males

\section{Jian Xiang}

Key Laboratory of Evidence Science, University Of Political Science and Law, Ministry of Education, Beijing, China 


\section{Purpose}

To investigate the waveform characteristics of optimized fourchannel mfVEP with normal gaze and experimental scotoma in Chinese young males.

\section{Methods}

MfVEPs with four bipolar channels were performed using the RETI-Port/Scan system (Roland Consult, Germany). The study included 30 healthy young males (51 eyes). The age range was 23-35 years. 51 eyes were measured with full field stimulation, and 30 eyes randomly chosen were measured with experimental scotoma (inferior-temporal quadrant, outer three rings area or two sectors covered). A pattern reversal dartboard

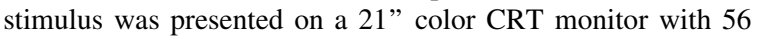
sectors (maximal eccentricity $25^{\circ}$ ) and two additional nasal sectors (up to $42^{\circ}$ ). Each sector had 16 checks, 8 white and 8 black. Luminances of the checks were $120 \mathrm{~cd} / \mathrm{m}^{2}$ (white) and $0 \mathrm{~cd} / \mathrm{m}^{2}$ (black) (Michelson contrast $99 \%$ ). Stimuli had a repetition rate of $60 \mathrm{~Hz}$ and the pattern of reversals followed a pseudo-random sequence. Visual stimuli were presented in four cycles of $140 \mathrm{~s}$ each. The patient was instructed to watch the central fixation mark (red cross). During each cycle, the patient's visual fixation was monitored with a video camera to ensure adequate cooperation. VEPs were recorded at four different locations over the primary visual cortex by means of a cross-shaped electrode fixation device with two fixed and two horizontally jointed arms. Four channels were derived: $\mathrm{CH} 1$, vertical; $\mathrm{CH} 2$, horizontal; $\mathrm{CH} 3$, left oblique; and $\mathrm{CH} 4$, right oblique. Two active electrodes were placed along the vertical midline $5.5 \mathrm{~cm}$ above and $1 \mathrm{~cm}$ below the inion. Two additional active electrodes were placed $4 \mathrm{~cm}$ on either side. A forehead electrode placed at the glabella served as the ground electrode. This electrode configuration relative to the cortical electric dipole ensured large VEPs. Biosignals were recorded with $1020 \mathrm{~Hz}$ sampling frequency The data were processed with the custom-designed program of RETI-Port/ Scan which selects the largest VEP recorded on each channel for each of the 58 stimulus fields: this recording represents the response to the concerning visual field and forms a new channel (CH5; best VEP of CH1-4). Subsequent analysis of $\mathrm{CH} 5$ data was performed with regard to four quadrants, five rings and twenty locations using the reconstructed waveforms method. Peak-to-trough amplitude, latency and amplitude density from a 50-160 ms window were calculated. Peak-totrough amplitudes in different fields before and after covering were compared.

\section{Results}

(1) Amplitude densities at inferior-nasal quadrant (304.32 \pm $128.47 \mathrm{nV} / \mathrm{deg}^{2}$ ), inferior-temporal quadrant (274.96 \pm $116.05 \mathrm{nV} / \mathrm{deg}^{2}$ ) were greater than at superior- nasal quadrant $\left(194.12 \pm 78.03 \mathrm{nV} / \mathrm{deg}^{2}\right)$, superior-temporal quadrant (177.56 $\pm 64.91 \mathrm{nV} / \mathrm{deg}^{2}$ ), but the amplitude densities at the upper retina were no difference from that at the lower retina within a $10^{\circ}$ eccentricity. Latencies at superior-nasal quadrant showed a prolonged trend compared to superior-temporal quadrant or superior- nasal quadrant. (2) Amplitude densities were greatest at the central fovea $\left(240.36 \pm 96.60 \mathrm{nV} / \mathrm{deg}^{2}\right)$ and reduced peripherally $\left(1.83 \pm 0.73 \mathrm{nV} / \mathrm{deg}^{2}\right)$. (3) Amplitude densities of responses at the 20 locations ranged from $287.71 \pm 132.84$ $\mathrm{nV} / \mathrm{deg}^{2}$ to $1.76 \pm 0.73 \mathrm{nV} / \mathrm{deg}^{2}$, and latencies ranged from $104.38 \pm 7.33 \mathrm{~ms}$ to $96.75 \pm 8.00 \mathrm{~ms}$. (4) MfVEPs in covered areas were undetectable or obviously decreased in all subjects.

\section{Conclusions}

Our results suggest mfVEP reflect visual function at different visual field locations correctly and objectively. MfVEP is influenced by experimental scotoma and changes are consistent with the visual field covered. MfVEPs offer the possibility of performing an objective visual field assessment in forensic medicine identification.

\section{Oral Session 8: Retinal degeneration II}

\section{OS8: Oral paper 1}

Comparisons among annual measures of spectral domain OCT, full-field ERG and static perimetry in patients with RPGR-mediated X-linked retinitis pigmentosa (XLRP)

\section{Birch $D^{1}$, Locke KG ${ }^{1}$, Klein $M^{1}$, Wen $Y^{2}, \operatorname{Hood~} \mathrm{DC}^{3}$, Wheaton DKH ${ }^{1}$, Hoffman DR}

${ }^{1}$ Retina Foundation of the Southwest, Dallas, TX, USA, ${ }^{2}$ Baylor University Medical School, Houston, TX, USA, ${ }^{3}$ Columbia University, New York, NY, USA

\section{Purpose}

We recently reported that short-term repeat variability is low compared to the annual rate of change in the width of the inner segment ellipsoid zone (EZ) from spectral domain (SD) OCT in patients with retinitis pigmentosa (RP). To further test the validity of EZ width as an outcome measure for clinical trials, we used a within-subjects design to compare the yearly decrease in EZ width with the mean decrease in full-field cone ERG amplitude and the mean decrease in visual field total sensitivity. Methods

Measures were obtained from 44 patients with XLRP due to a mutation in the RPGR gene. Each patient had cone $31 \mathrm{~Hz}$ ERG amplitude $>1.0 \mu \mathrm{V}$ at baseline. On at least three consecutive yearly visits, SD-OCT scans were obtained with a Heidelberg Spectralis HRA + OCT (Heidelberg, Germany). Automatic real time registration was used with an average of 100 scans. Annual scans were captured with the aid of automatic registration to ensure the same scan placement on each visit. Manual segmentation was aided by subroutines written in Igor Pro 6.03A (WaveMetrics Inc., USA) based on previously published Matlab (Mathworks, Natick, MA) routines. Full-field ERGs (ISCEV protocol) were obtained with an Espion E2 (Diagnosys, Lowell, MA). Static perimetric fields were obtained with a Humphrey field analyzer (Carl Zeiss Meditec, Dublin, CA).

\section{Results}

The average yearly decrease in EZ width in patients with RPGR-mediated XLRP was $0.86^{\circ}(248 \mu \mathrm{m}-8 \%)$. Assuming equal decrease in each meridian, this translates to a $15 \%$ average rate of change in equivalent area. By comparison, the mean annual decrease in cone $31 \mathrm{~Hz}$ flicker amplitude was $8 \%$, while the annual decline in total field sensitivity was $7 \%$. Within each patient, analyses of segmented retinal layer thicknesses show positive interrelationships between structural and functional measures of progression. 


\section{Conclusions}

EZ width declines rapidly in patients with RPGR-mediated XLRP ( $8 \%$ annually). Moreover, unlike cone ERG amplitude and visual field sensitivity, EZ width repeat variability is less than the annual rate of change. These two advantages support the use of EZ width as an outcome measure in prospective clinical trials in RP.

\section{OS8: Oral paper 2}

\section{RDH5 retinopathy (fundus albipunctatus) with normal rod ERGs in Chinese patients}

\section{Sui Ruifang, Xiaowei Liu, Liang Liu, Hui Li, Fei Xu, Ruxin Jiang}

Ophthalmology, Peking Union Medical College Hospital, Beijing, China

\section{Purpose}

The aim of this study was to characterize the clinical features of four unrelated Chinese patients with retinol dehydrogenase 5 (RDH5) retinopathy (fundus albipunctatus) and to identify the genetic defects underlying this disorder.

\section{Methods}

Complete ophthalmic examinations including slit-lamp biomicroscopy, dilated indirect ophthalmoscopy, spectral domain OCT (SD-OCT), and full-field ERGs were performed. Genomic DNA was prepared from peripheral venous leukocytes. Polymerase chain reaction and direct sequencing were used to screen the coding exons and exon/intron boundaries of the RDH5 gene (11-cis-retinol dehydrogenase).

\section{Results}

Four patients with RDH5 retinopathy, including two 6-year-old boys, from four unrelated Chinese families were recruited in this study. A novel c.1015C > T (p.Arg278Ter) nonsense mutation of the RDH5 gene was identified in one 6-year-old boy, who has a compound heterozygous mutation with c.928delC/InsGAAG (p.Leu310GluVal). Homozygous Leu310GluVal mutations were identified in two male patients including the other 6-year-old boy. The other patient was a 29-year-old woman, in whom compound heterozygous changes c.683G $>\mathrm{A}$ (p.Arg167His) and Leu310GluVal in RDH5 were identified. All patients manifested the fundus phenotype of fundus albipunctatus. ERGs recorded in the two boys showed scotopic waveforms within the normal range, while higher amplitudes were recorded after prolonged dark adaptation. The two adult patients had decreased scotopic ERG responses under standard conditions. OCT showed discrete highly reflective lesions extending from the retinal pigment epithelium to the level of the external limiting membrane.

\section{Conclusions}

Novel c.1015C > T (p.Arg278Ter) nonsense mutation in RDH5 was identified. Leu310GluVal mutations were frequently related to Chinese patients with RDH5 retinopathy. Normal scotopic ERG responses were observed in this study.

\section{OS8: Oral paper 3}

Identification of the CHM gene mutation in a Chinese family with choroideremia
Yan Teng, Xubo Mengqingfeng, Sunqing Tengyufei

Affiliated Ophthalmology Hospital, the First Clinical

College of Harbin Medical University, Harbin City, China

\section{Purpose}

To identify the mutation in the CHE gene in a Chinese fourgeneration family with choroideremia (CHM).

\section{Methods}

Patients and family members were given complete examinations. Genomic DNA was extracted from leukocytes of venous blood of 15 individuals in the family and 180 healthy Chinese individuals. All of the 15 coding exons were amplified in the proband by polymerase chain reaction and followed by direct sequencing. Protein structure was modeled based on the protein data bank and mutated in DeepView v4.0.1 to predict the effect of the mutation.

Results

All affected male adults had characteristic signs and symptoms of CHM, decreased visual acuity (VA) in the first and third decades. Best-corrected visual acuity of the affected males was 0.43 (0.3 to $0.6)$. Follow up at 3 years showed signs of decreased vision $0.11(0.01-0.3)$. Three year rate of VA change was 0.32 . In affected male children, fundus has only mild peripapillary sclerosis and peripheral retinal pigment epithelium mottling. One older affected female showed classic choroideremia, decreased visual acuity in the fifth decade, best-corrected visual acuity of 0.5OD and 0.25OS. Follow up at 3 years showed signs of decreased vision (0.2OU). Three year rate of VA change was 0.35 . One young female carrier did not have any symptoms, but her fundus demonstrated a conspicuous pattern of retinal pigment epithelium mottling, mainly located at the peripheral retina, while another older female carrier did not show any signs or symptoms. A novel heterozygous c. $1837 \mathrm{G}>$ A change in exon 15 of CHM was detected in the proband, which resulted in the substitution of serine by proline at codon 613 (p.D613 N). This mutation was also present in six family members but absent in the others and the 180 healthy Chinese individuals. Based on molecular modeling, the misfolded protein caused by the mutation might destabilize the structure of the helix that potentially could affect the global stability of the Rep-1 protein. Conclusions

Chorioretinopathy of $\mathrm{CHE}$ originate from peripapillary and peripheral. Chinese male patients with CHM may experience severe vision loss at an earlier age, whereas female CHM carriers may manifest signs and symptoms at a later age, but a fast rate of VA loss and the prognosis for central VA. The difference in phenotype of female carriers is not only related to age. A novel p.D613 N mutation was identified in the CHM gene, which may be the causative mutation for choroideremia in this family.

\section{OS8: Oral paper 4}

More morphological details in autosomal recessive bestrophinopathy (ARB)

Wildberger JGH ${ }^{1}$, Berger $\mathbf{W}^{2}$, Bahr $\mathrm{A}^{2}$, Hanson $\mathbf{J}^{1}$, Gerth-Kahlert $\mathbf{C}^{1}$

${ }^{1}$ Department of Ophthalmology, University Hospital Zurich, Switzerland, ${ }^{2}$ Institute of Medical Molecular 
Genetics, University of Zurich, Zurich-Schwerzenbach, Switzerland

\section{Purpose}

To describe morphological features in a small clinical case series with bestrophin 1 retinopathy.

\section{Methods}

Visual dysfunction of variable severity was demonstrated with retinal electrophysiology (ERG, mfERG). Fundus photographs were taken including OPTOS-wide angle, near-infrared (NIR) and autofluorescence (AF). A detailed spectral domain OCT exploration with quantitative segmentation of retinal layers was performed at the posterior pole and near-periphery including an en face/ transverse display of the foveolar region (Heidelberg 261 scan lines, $\mathrm{HR}$, distance between scans 11 micrometers, test area $10 \times 15$ degrees-of-arc). In addition, circumpapillary RNFL measurements including the papillomacular bundle (PMB) were obtained. Genetic analyses were performed on DNA extracted from blood samples and Sanger DNA sequencing for all exons of the gene.

\section{Results}

The identification of bi- and mono-allelic mutations in the BEST1 gene supported the clinical diagnosis of recessive or dominant bestrophin-associated retinopathies in all patients. The OPTOS photography disclosed a diffuse increase of AF across the posterior pole indicating a retinal involvement extending beyond the arcuate retinal vessels. Localized subretinal multiple deposits of high reflectivity and of increased AF in addition to the diffusely increased AF, were scattered either in the fovea and/or in the retinal near-periphery at the RPE layer level and followed also the peripheral border of the presumed advanced structural damage in form of pearl-string arrangements. The enface imaging demonstrated localization and extent of these deposits. The retina was elevated in the foveola with the creation of a subretinal slit-like space. These cavities found their peripheral continuation in form of a marked thickness gain of the outer photoreceptor segment and of a structural loss of the 3 bands. This space was not empty, eventually contained retinal structures like rosettes (tubulations of photoreceptor elements). A disappearance of the RPE was questionable at the posterior pole, since the degree of enhanced reflectivity from the underlying choroid was often less pronounced compared with atropic areas of Stargardt maculopathy or RP. The outer nuclear layer $(\mathrm{ONL})$ as measured from the external limiting membrane (ELM) to the outer plexiform layer (OPL) showed a widespread thickness loss. The inner nuclear layer (INL) was thicker than normal and contained cavities. Retinal ganglion cell layer (GCL) and RNFL demonstrated tendency for an increased thickness as well, the latter mainly in the PMB. The normally contourless inner plexiform layer (IPL) was easier separated from CGL in ARB.

\section{Conclusions}

Morphological alterations typical for bestrophin retinopathy should be separated from unspecific alterations appearing in other retinal dystrophies as well. The following changes may be directly related to bestrophin retinopathy: these are the highly reflective circumscribed deposits in the subretinal foveolar seemingly empty space and towards the periphery continuing structually-deprived and markedly thickened outer photoreceptor segment. The pearl-string-like punctual arrangement of increased AF, which follows the outer borders of the main retinal damage in the near-periphery seems to be bestrophin retinopathy-specific as well. The recently introduced en-face OCT imaging furnishes an improved insight into the size and distribution of the outer-retinal deposits and of the INL-cavities.

\section{OS8: Oral paper 5}

Phenotypic and genotypic characterization of Bietti crystalline dystrophy

Holder $\mathrm{GE}^{1,2}$, Liew $\mathbf{G}^{\mathbf{1}}$, Halford $\mathrm{S}^{\mathbf{3}}$, Mackay $\mathrm{D}^{\mathbf{1}}$, Sergouniotis $P^{1,2}$, Holt $R^{3}$, Broadgate $S^{3}$, Ocaka $L^{4}$, Robson $A^{1,2}$, Moore $A^{1,2}$, Webster $A^{1,2}$

${ }^{1}$ Moorfields Eye Hospital, London, UK, ${ }^{2}$ UCL Institute of Ophthalmology, London, UK, ${ }^{3}$ University of Oxford, UK, ${ }^{4}$ Institute of Child Health, London, UK

\section{Purpose}

To provide a detailed phenotype/genotype study of Bietti crystalline dystrophy (BCD).

\section{Methods}

Observational case series. Twenty patients recruited from a multi-ethnic British population. Patients underwent colour fundus photography, near infra-red (NIR) imaging, fundus autofluorescence (AF) imaging, spectral domain (SD) OCT and ERG assessment. The gene CYP4V2 was sequenced.

Results

Patients ranged in age from 19 to 72 years (mean 42.1), with visual acuity of $6 / 5$ to perception of light (mean 6/15). There was wide intra- and inter-familial variability in clinical severity. Retinal crystals were not evident on fundus AF imaging, and were best visualized with near infra-red imaging. SD-OCT showed them to be principally located on or in the retinal pigment epithelium (RPE)/Bruch's membrane complex. Disappearance of the crystals, revealed by serial recording, was associated with severe disruption and thinning of the RPE/Bruch's membrane complex. Cases with extensive RPE degeneration ( $\mathrm{N}=5$ ) had ERGs consistent with generalized rod and cone dysfunction, but those with more focal RPE atrophy either showed amplitude reduction without delay $(\mathrm{N}=3$ ), consistent with restricted loss of function, or were normal $(\mathrm{N}=2)$. Seven novel likely disease-causing variants were identified with the most common variant in the cohort being the novel p.Met66Arg, found in all 11 patients of South Asian descent. This mutation appears to be associated with earlier onset (median age 30) compared to other substitutions (median age 41). Deletions in exon 7 were associated with more severe disease.

\section{Conclusions:}

The phenotype is highly variable. Several novel variants are reported, including a highly prevalent substitution in patients of South Asian descent associated with earlier onset disease. Crystals were generally situated on or in the RPE/Bruch's complex, supporting a role for the RPE in disease pathogenesis, but could disappear over time with associated RPE disruption.

\section{Oral Session 9: Vessels and toxicology}

\section{OS9: Oral paper 1}

Study on the correlations of visual acuity and OCT with mfERGs in diabetic macular edema 


\section{Zhen Tian, Wu Pei-pei, Huang Shi-zhou, Luo Guang-wei, Jin Chen-jin}

Zhong Shan Ophthalmic Center of Sun Yat-sen University, Guangzhou, China

\section{Purpose}

To investigate the correlations of best correct visual acuity (BCVA), OCT and mfERG in patients with diabetic macular edema and to analyze the relationship of visual acuity, morphological changes and retinal function.

\section{Methods}

Nineteen patients (38 eyes) with diabetic macular edema in severe non-proliferative stage of diabetic retinopathy participated in the study. Veris mfERG measurement and Heidelberg OCT examination were performed. Correlations among values of latent period (lp) and response densities (rd) of the mfERG components and macular retina thickness (within $1 \mathrm{~mm}$ area, 1-3 $\mathrm{mm}$ annular area and 3-6 $\mathrm{mm}$ annular area) as well as BCVA were evaluated by software SPSS 13.0.

\section{Results}

The average macular retina thickness was positively correlated with the lp of N1, P1, N2 at three separate regions $(\gamma=0.535-0.754$, $P<0.05)$. The correlation coefficient for N1 was higher than for $\mathrm{P} 1$, and was lowest for N2 ( $\gamma \mathrm{N} 1-\mathrm{rd}>\gamma \mathrm{P} 1-\mathrm{rd}>\gamma \mathrm{N} 2-\mathrm{rd})$. In the three regions, the correlation coefficient between lp of N1, P1, N2 and average macular retina thickness descended in 1 mm, 1-3 mm and 3-6 mm annular area. BCVA was positively correlated with the response densities of $\mathrm{N} 1, \mathrm{P} 1, \mathrm{~N} 2$ in every region $(\Gamma \mathrm{P} 1=0.263-0.770, \quad P<0.05 ; \quad \Gamma \mathrm{N} 1=-0.611$ to -0.797 , $P<0.05)$. The correlation coefficient increased gradually from inside to outside regions ( $\gamma 1 \mathrm{~mm}>\gamma 1-3 \mathrm{~mm}>\gamma 3-6 \mathrm{~mm})$.

\section{Conclusion}

In the severe non-proliferative stage of diabetic retinopathy, retinal function is affected by macular retina thickness. Within the $6 \mathrm{~mm}$ macular region, the correlation coefficient is higher with closer distance.

\section{OS9: Oral paper 2}

Fundus autofluorescence imaging of acute zonal occult outer retinopathy

\section{Qian Wang, Libin Jiang}

Eye Center, Beijing Tongren Hospital, Capital Medical University, Beijing Ophthalmology \& Visual Sciences Key Lab, Beijing, China

\section{Purpose}

To investigate fundus autofluorescence (FAF) findings in eyes with acute zonal occult outer retinopathy (AZOOR).

\section{Methods}

Retrospective analysis of FAF findings in a series of patients with AZOOR, and characterisation of FAF manifestations and the relationships between FAF and OCT, Humphrey visual field tests (HVF), mfERG and full field ERG are discussed.

\section{Results}

There were 20 eyes of 20 patients (16 women, $80.0 \%, 4$ men, $20.0 \%$ ), who had a mean age of $32.14 \mathrm{yr}(9-57 \mathrm{yr})$, diagnosed with AZOOR according to the diagnostic criterion proposed by Gass. In all patients unilateral involvement was found (100\%). At first presentation, the best corrected visual acuity ranged from $2 / 200$ to $24 / 20$. Non-contact tonometer tests were all within normal limits. Anterior segment examinations were normal, except the presence of relative afferent papillary defect (RAPD) in $9(45.0 \%)$ of the affected eyes. FAF abnormalites were detected in 18 of 20 eyes $(90.0 \%$ ), and all of them showed broad areas of hyperautofluorescence. OCTs, HVFs, and mfERG/ERGs were obtained from all of the 20 eyes. OCT revealed an absent inner segment/outer segment (IS/OS) boundary corresponding to the areas of dysfunction demonstrated by mfERG and HVF. In most patients, abnormalities found on FAF corresponded to the abnormalities demonstrated in HVF, mfERG and OCT.

Conclusions

FAF abnormalities were detected in most AZOOR eyes, and some of them corresponded to the dysfunctional areas revealed by OCT, HVF and mfERG/FERG. It was suggested that FAF might be used to diagnose AZOOR.

\section{OS9: Oral paper 3}

\section{Non-mydriatic diabetic retinopathy screening using} $30 \mathrm{~Hz}$ flicker ERG with skin electrodes

\author{
Davis $\mathbf{Q}^{1}$, Severns $\mathbf{M}^{1}$, Johnson $\mathbf{M}^{2}$, Liss $\mathbf{R}^{2}$ \\ ${ }^{1}$ LKC Technologies, Inc., Gaithersburg, MD, USA, \\ ${ }^{2}$ University of Maryland, Baltimore, MD, USA
}

\section{Purpose}

Diabetic retinopathy (DR) is one of the leading causes of vision loss in working-age adults, causing 12,000 to 24,000 new cases of blindness annually in the United States (CDC National Diabetics Fact Sheet, 2007). The $30 \mathrm{~Hz}$ flicker ERG implicit time has been shown to correlate with the extent of DR (Bresnick and Palta 1987; Satoh et al. 1994; Han and Ohn 2000). Nevertheless, the use of the ERG for DR screening has been limited because of, among other reasons, the use of corneal-contact electrodes, mydriatic eye drops, long test durations, and the difficulty in interpreting ERG results. A proof-of-principle prototype DR screener that eliminates the above-mentioned problems was developed, and its performance in a small population of diabetics is described.

\section{Methods}

An LKC UTAS SunBurst ganzfeld was modified by adding an ArringtonResearch ViewPoint EyeTracker. The pupil diameter was measured before the start of data collection to set the desired retinal illuminance. Pupil diameters were assumed to be constant during the test. Pediatric ECG electrodes manufactured by Nikomed (Huntingdon Valley, PA, USA) were attached to the skin to measure the retina's response. Using an IRB-approved protocol, 36 humans were tested after informed consents were obtained. Stimuli were $30 \mathrm{~Hz}$ flickering light having 1, 3.2, 10, and $32 \mathrm{Td}$-s flash retinal illuminance energies with and without a $1500 \mathrm{Td}$ background. Not all subjects were tested with each stimulus. $1500 \mathrm{Td}$ is equivalent to the ISCEV standard background luminance of $30 \mathrm{~cd} / \mathrm{m}^{2}$ through an $8 \mathrm{~mm}$ pupil. A retinal specialist (Liss) determined each subject's level of DR using fundus photos that included the posterior pole, the $\mathrm{ONH}$, and peripheral views as well as for some subjects a 
comprehensive eye exam. Performance was characterized by sensitivity and specificity to vision-threatening DR, which is defined as severe non-proliferative DR and worse or equivalently, as an ETDRS level of 53 or greater.

\section{Results}

The background light reduced the changes seen in implicit times from increasing levels of DR; results described below have the background light off. Using $10 \mathrm{Td}$-s flashes, a sensitivity of $67 \%(2 / 3)$ and a specificity of $86 \%$ (30/35) was obtained. Using $3.2 \mathrm{Td}$-s flashes was an improvement, having a sensitivity of $100 \%(3 / 3)$ and a specificity of $83 \%$ (29/35). Using 1 Td-s flashes provided complete separation of the populations, with a sensitivity of $100 \%(2 / 2)$ and a specificity of $100 \%(34 / 34)$. Using a combination of intensities provided better results. Using $3.2 \mathrm{Td}-\mathrm{s}$ and $10 \mathrm{Td}-\mathrm{s}$, a sensitivity of $100 \%(3 / 3)$ and a specificity of $89 \%(31 / 35)$ was obtained. Using 1 Td-s and 10 Td-s has a sensitivity of $100 \%(2 / 2)$ and specificity of $100 \%$ (34/34) with greater separation than using 1 Td-s alone.

\section{Conclusions}

$30 \mathrm{~Hz}$ flicker ERG testing can be used to screen for visionthreatening DR, without the need to dilate the eye and without the need to contact the eye with electrodes. Sensitivity and specificity using the flicker ERG is better than 1-field and 2-field fundus photography with mydriasis, and non-dilated wide-field scanning laser ophthalmoscopy (WSLO), all of which have sensitivities in the range of $65-69 \%$ and specificities in range of $83-88 \%$ (Wilson et al. 2010). The device and electrodes used to obtain these results have been re-engineered into the LKC RETeval device and sensor strips to provide an easy-to-use platform for performing $30 \mathrm{~Hz}$ flicker testing for DR screening and other applications.

Acknowledgment: The project was supported by Award Number R43MD003432 from the National Center on Minority Health and Health Disparities. The content is solely the responsibility of the authors and does not necessarily represent the official views of the sponsor.

\section{OS9: Oral paper 4}

\section{Sensitivity and specificity of mfERG in screening for hydroxychloroquine/chloroquine induced retinopathy}

\section{Coupland S, Gottlieb C, Leonard K, Gao J, Hamilton J}

University of Ottawa Eye Institute, Ottawa, ON, Canada

\section{Purpose}

Hydroxychloroquine (HCQ) and chloroquine (CQ) are commonly prescribed medications for the treatment of certain rheumatic and autoimmune diseases. While toxicity is uncommon it can cause a progressive loss in vision and irreversible retinopathy and is a widely recognized complication of long term HCQ and CQ therapy. The purpose of this study was to evaluate the effectiveness of the mfERG in detecting HCQ/CQ induced retinopathy in comparison to diagnosis by an expert panel consensus, which acts as a reference standard representative of the clinical setting.

\section{Methods}

A retrospective chart review of 165 patients on HCQ or CQ therapy referred to the University of Ottawa Eye Institute between
2007 and 2011 for mfERG was undertaken. Seven patients were excluded due to unrelated pre-existing retinopathy. All mfERG tests were interpreted by an experienced clinical electrophysiologist. An expert panel independently assessed patients for the development of retinal toxicity using the composite results of serial clinical exams, visual field testing (Humphrey 10-2), and time- or spectral-domain OCT. Any discrepancies were resolved by a meeting of the expert panel. Comparison was then made between the assessment of toxicity based on the expert panel findings, and the interpretation of the mfERG results.

\section{Results}

The expert panel assessment identified a total of 28 of 158 (17.7\%) patients who developed some evidence of retinal toxicity during follow-up. In $25.0 \%$ of the cases, the mfERG detected retinopathy prior to the reference test, suggesting higher sensitivity. In another $57.1 \%$ of cases, the mfERG confirmed the retinal toxicity previously detected by the reference test. The mfERG had false negative detection in $17.9 \%$ of cases. Of the 130 patients in whom no retinopathy was detected by the reference test, $70.0 \%$ were confirmed by mfERG. In the other $30.0 \%$, the mfERG revealed some level of retinopathy, which suggests either false positive detection or higher sensitivity. The mfERG had a sensitivity of $82.1 \%$ and specificity of $70.0 \%$ for detection of $\mathrm{HCQ} / \mathrm{CQ}$ induced retinopathy.

\section{Conclusions}

Our results demonstrate the utility of mfERG in screening for HCQ/CQ induced retinopathy. The mfERG may be more sensitive for the early detection of retinal changes associated with these medications, as compared to the current standard of clinical examination and automated perimetry.

$\mathrm{S}$ Coupland is a consultant to Diagnosys LLC

\section{OS9: Oral paper 5}

Early signs of hydroxychloroquine and chloroquine toxicity in patients with autoimmune connective tissue diseases: two years follow up with multifunctional tests

Ruberto $G^{1}$, Cavagna $L^{2}$, Raimondi $M^{1}$, Mogavero $V^{1}$, Piccinini $\mathbf{P}^{1}$, Milano $\mathbf{G}^{1}$, Bianchi $\mathbf{P E}^{1}$

${ }^{1}$ Ophthalmic Clinic IRCCS, S. Matteo Hospital, Pavia, Italy, ${ }^{2}$ Rheumatology Clinic IRCCS, S. Matteo Hospital, Pavia, Italy

\section{Purpose}

To follow up early functional and morphologic signs of hydroxyhcloroquine (HCQ) and chloroquine (CQ) toxicitytime and dose effects.

\section{Methods}

We present a 2-year follow-up of 63 patients (53 females, 10 males, mean age $55.4 \pm 13.6 \mathrm{yr}$ ) affected by various autoimmune tissue diseases (i.e. rheumatoid arthritis, systemic lupus erythematosus, connective tissue diseases and others). The patients, who had undertaken therapy for $0-5,5-10$ and $>10$ years respectively, were examined using multiple testing procedures, including a complete ophthalmologic visit, logMAR visual acuity (VA), mfERG (63 hexagons), frequency doubling technology (FDT), automated perimetry (AP, program 10-2), Spectralis OCT and autofluorescence (FA). The 
average amount of drug administered was $147.1 \pm 143.2$ g. For the statistical analysis, we considered the latencies and amplitudes of the 5 mfERG rings, the pattern and mean deviation (PSD, MD) results of the FDT and AP, the fiber thickness (FT) of the OCT and the FA positivity or negativity. All the tests were performed two times, at baseline and one year later.

\section{Results}

We found multiple statistically significant results in the mfERG measurements, both in amplitudes (mainly) and latencies: the latency increased in the first ring from $14.83 \mathrm{~ms}$ to 15.52 $(p=0.04)$. The amplitudes of $\mathrm{N} 1$ and $\mathrm{P} 1$ waves decreased in nearly all the rings: N1 from -244.86 to $195.70(p=0.002), \mathrm{N} 2$ from -170.40 to $142.48(p=0.005)$, N5 from -150.99 to $-136.22(p=0.05)$. Variations in $p$ amplitudes between the first and the second control were: P1 608.20-522.34 ( $p=0.0001)$, P2 491.55-431.68 $(p=0.0001)$, P3 451.81-401.02 $(p=$ $0.0001), \mathrm{P} 4459.57-417.81$ ( $p=0.0009), \mathrm{P} 5472.61-424.57$ ( $p=$ 0.0001). The PSD in both AP and FDT did not change significantly, while paradoxically the MD improved from -1.32 to 0.96 and from 0.94 to 0.15 respectively ( $p=0.005$ and 0.0008 ). Interestingly, OCT also showed a variation in fiber thickness: more precisely, a thickening in various sectors, which was statistically significant in two of these sectors: the nasal sector (from 320.42 to $325.97(p=0.04)$ ), and the peripheral inferior sector (from 277.83 to $284.61(p=0.01)$ ).

\section{Conclusions}

MfERG seems to be the best functional test to follow up early changes in the retina of patients in treatment with HCQ and $\mathrm{CQ}$. The AP and FDT bias probably reflect a learning effect. Finally, the OCT suggests a correlation between functional and morphological effects of the considered drugs.

\section{OS9: Oral paper 6}

Using the mfERG to evaluate the prevalence, onset, and progression of hydroxychloroquine retinal toxicity: a review of 90 cases

\section{Lyons JS}

Georgetown University/Washington Hospital Center Program in Ophthalmology, Silver Spring, MD, USA

\section{Purpose}

To show that hydroxychloroquine (HCQ) retinal toxicity is not a rare event and is becoming more prevalent with long-term use and increasing cumulative dose. To further confirm the time course of retinal toxicity defining an initial relatively sudden loss of visual function, which appears to be arrested or show limited progression once the medication is discontinued.

\section{Methods}

We have now evaluated over 600 patients referred for screening for plaquenil toxicity. Screening included visual acuity, Humphrey visual fields, slit lamp examination, fundus examination, digital photography, mfERG using the Veris 61 hexagon 4 min test strategy, and full field ERGs where indicated. Spectral domain (SD) OCT was more recently added. We analyzed the clinical data as well as the full field and mfERG data using ring ratio analysis including comparison of ring 1 to ring 2 and ring 2 to ring 5 to define the loss of paracentral function.

\section{Results}

Of the more than $600 \mathrm{HCQ}$ patients screened, we found 90 patients with HCQ retinal toxicity as defined by ring ratio analysis, visual field defects and clinical characteristics. All but 7 of these patients had taken more than 1,000 $\mathrm{g}$ of HCQ cumulative dose. Of those 7, three had taken CQ in addition to HCQ, one had liver disease, and two others received a higher than standard daily dose. While it is difficult to derive an accurate populational prevalence of HCQ retinal toxicity, our data shows a prevalence of approximately $15 \%$ of all patients screened and of over $30 \%$ for patients who have taken more than $1,000 \mathrm{~g}$ cumulative. Estimate of the time of onset of toxicity to the time of the patient evaluation confirms toxicity occurs between 6 and 18 months based on earliest reported symptoms and/or prior visual fields or mfERGs where available. In the patients on HCQ who initially showed deficits limited to the central and/or paracentral regions, there was no loss of visual acuity and only minor loss of visual function as measured by Humphrey 10-2 visual fields or mfERG in follow-up ranging from 6 months to 9 years.

\section{Conclusion}

This confirms our earlier findings that HCQ toxicity increases significantly with increasing cumulative dose. Since many of these patients were referred for evaluation of visual field defects, our prevalence data is likely to be skewed but not to the extent that toxicity can be described as a rare occurrence. Our estimate of 6-18 months from time of onset to confirmation of toxicity validates the recommendation that annual testing, accompanied by immediate reporting of changes in vision, should be effective in detecting early stages of toxicity. Our findings also indicate that stopping the drug upon detection of toxicity by screening with visual fields and confirming toxicity with mfERG measuring paracentral defects with two complementary ring ratio tests appears to prevent significant further loss of visual function at least in the near to intermediate term.

\section{Poster Session A}

\section{PS A: Poster A1}

Mueller cell sheen dystrophy - 10 year clinical and electrophysiological follow-up

Kellner $\mathbf{U}^{1,2,3}$, Renner $\mathrm{AB}^{3,4}$

${ }^{1}$ RetinaScience, Bonn, Germany, ${ }^{2}$ Zentrum für seltene Netzhauterkrankungen, AugenZentrum Siegburg, Siegburg, Germany, ${ }^{3}$ Klinik und Poliklinik fuer Augenheilkunde, Charite Universitaetsmedizin Berlin, Berlin, Germany,

${ }^{4}$ Klinik und Poliklinik für Augenheilkunde,

Universitaetsklinikum Regensburg, Regensburg, Germany

\section{Purpose}

To describe the clinical and electrophysiological data of a patient with Mueller cell sheen dystrophy (MCSD) during progression.

\section{Methods}

Examinations included best corrected visual acuity (BCVA), kinetic perimetry, biomicroscopy, funduscopy, fundus photography, fluorescein angiography, fundus autofluorescence (FAF), OCT, full-field ERG and mfERG. 


\section{Results}

A 61-year-old woman presented with folds of the internal limiting membrane (ILM) at the posterior pole of both eyes, and cystoid macular edema (CME) in the right eye. After 3 and 9 years, always a rapid and distinct visual loss appeared along with CME, increase of folds, cystoid spaces intraretinal and subretinal fluid. During 10 years, BCVA decreased from 0.2 to $1 / 15$ at OD and from 0.7 to hand movements at OS. ERG showed negative waveforms with ongoing amplitude reduction at OD over time and was normal at OS over 8 years.

Conclusions

MCSD presents with late onset, ILM folds, cystoid spaces in all retinal layers and a negative ERG.

\section{PS A: Poster A2}

Combined confocal near-infrared reflectance $(815 \mathrm{~nm})$ and macular spectral domain OCT identify optic atrophy in patients with bilateral unexplained visual loss

Kellner $\mathbf{S}^{1,2}$, Neuhann $\mathbf{T M}^{3}$, Abicht $\mathrm{A}^{3}$, Wissinger $\mathrm{B}^{4}$, Renner $\mathbf{A B}^{5}$, Weinitz $\mathrm{S}^{1,2}$, FarmandG ${ }^{1}$, Kellner $\mathrm{U}^{1,2}$,

${ }^{1}$ Zentrum seltene Netzhauterkrankungen, AugenZentrum Siegburg, Siegburg, Germany, ${ }^{2}$ RetinaScience, Bonn, Germany, ${ }^{3}$ Medizinisch Genetisches Zentrum, Muenchen, Germany, ${ }^{4}$ Molekulargenetisches Labor Tuebingen, Zentrum für Augenheilkunde, Universitaetsklinik Tübingen, Tuebingen, Germany, ${ }^{5}$ Klinik und Poliklinik für Augenheilkunde, Universitaetsklinikum Regensburg, Regensburg, Germany.

\section{Purpose}

To report distinct phenomena in near-infrared reflectance (NIR) and spectral domain (SD-OCT) indicative of optic atrophy in patients with unexplained visual loss.

\section{Methods}

In a consecutive series of 426 patients referred to a tertiary care center for evaluation of unexplained visual loss a group of 21 unrelated patients with optic atrophy were identified. In addition to clinical eye examination they underwent combined NIR and SD-OCT imaging (Spectralis OCT, Heidelberg Engineering, Germany), as well as fundus and near-infrared autofluorescence (FAF, NIA; HRA2, Heidelberg Engineering, Germany). MfERG or VEPs according to ISCEV standards as well as molecular genetic evaluation were performed in the majority of patients. Results

All 21 patients complained about variable loss of visual function. The majority of patients were referred after previous diagnostic processes were inconclusive. Outer retinal disorders were excluded based on normal retina on ophthalmoscopy, normal outer layers in the SD-OCT, normal FAF and NIA and normal mfERG in 14/14 examined patients. In 12/12 examined patients VEP latency was either delayed or amplitudes were not measureable. In all patients macular SD-OCT was suspicious of optic atrophy based on marked thinning of the nerve fiber layer. In addition, all patients presented with areas of reduced reflectance in NIR in the macula. Molecular genetic evaluation revealed four patients with OPA1 mutations, one patient with an m.14495 mutation, was denied by six patients and was without results in nine patients. In five patients without mutations family history indicated additional affected family members.

\section{Conclusion}

Confocal NIR and macular SD-OCT imaging may present valuable and readily identifiable signs indicative of optic atrophy facilitating the diagnostic process for patients and professionals.

\section{PS A: Poster A3}

The follow-up of a case of vitelliform macular dystrophy over an 8 year period

Huang Shizhou, Lezheng Wu, Feng Wen, Guangwei Luo, Futian Jiang

Zhongshan Ophthalmic Center, Sun Yat-sen University; State Key Laboratory of Ophthalmology, Guangzhou, China

\section{Purpose}

To show the follow-up of a case of vitelliform macular dystrophy with morphological and visual functional tests over an 8 year period.

\section{Methods}

The morphological examination included color photography, fluorescein angiography, OCT and the visual functional tests included visual acuity, EOG, mfERG. These were observed in a case of vitelliform macular dystrophy during the follow-up period from 2003 to 2011 .

\section{Results}

During the follow-up, color photography and fluorescein angiography showed that the sensory retina detachment and yellow-white deposits were improved. OCT examination showed that the sensory retinal detachment and subretinal hyper reflective deposits were decreased, which corresponded with the changes in fluorescein angiography. Visual acuity stayed the same during the follow-up. Although the Arden ratio of EOG was decreased, the amplitudes of mfERG had slightly increased. Conclusions

The retinal morphological changes and visual function have slight improved in this case of vitelliform maculopathy. The prognosis is good.

\section{PS A: Poster A4}

The clinical application of PERG in macular diseases

Wu Dezheng", Li Meng yuan², Yao Cuiqun², Gao Rulong², Liang Jiongji ${ }^{1}$

${ }^{1}$ Zhongshan Ophthalmic Center, Sun Yat-sen University, Guangzhou, China, ${ }^{2}$ Guangzhou Aier Eye Hospital, Zhongnan University, Guangzhou, China

\section{Purpose}

To evaluate the clinical application value of PERG for visual function in macular diseases.

\section{Methods}

According to ISCEV standard for clinical pattern electroretinography (PERG): 2012 update methods, 75 normal cases (150 
eyes) were tested, one eye randomly selected from each case, and divided into two groups on the basis of age: one group for 10-44 yr (45 cases, 45 eyes, 20 males and 25 females, visual acuity greater than or equal to 1.0), the another group for 45-74 yr (20 cases, 20 eyes, 8 males and 12 females, visual acuity greater than or equal to 0.8 ). By statistics, median and 5-95\% confidence limits were determined, which defined normal reference values. PERG testing was performed in the 21 patients (27 eyes) with macular diseases including age-related macular degeneration, pathologic myopia, macular atrophy, macular hole and diabetic macular edema and other diseases, aged from 10 to $76 \mathrm{yr}$ and with visual acuity from 0.1 to 0.5 . With reference to normal values of the control group, PERG abnormalities were determined in the macular disease patients. Results

The measured components included N35, P50 and N95 peak times, P50-N35 amplitude, N95-P50 amplitude with 4rps stimulation, and response amplitude with 15rps stimulation. In the macular disease group aged less than $45 \mathrm{yr}, 100 \%$ of eyes had abnormal P50-N35 amplitude with 4rps stimulation, and $100 \%$ of amplitudes with $15 \mathrm{rps}$ stimulation were abnormal. The abnormal rates of other parameters varied. In the macular disease group aged greater than or equal to $45 \mathrm{yr}$, $100 \%$ of eyes had abnormal N35 peak times and P50-N35 amplitudes with 4rps stimulation and $100 \%$ of amplitudes with $15 \mathrm{rps}$ stimulation were abnormal. The abnormal rates of other parameters varied.

\section{Conclusion}

The PERG is a relatively simple, fast and secure way for testing macular function and can determine visual functional abnormalities in patients with macular diseases, especially for the elderly, children and other patients. Therefore PERG should be widely applied.

\section{PS A: Poster A5}

Retinal function in severe and resistant retinoblastoma, and the effect of superselective intra-arterial chemotherapy

Tsapenko I, Zueva M, Saakyan S, Jarwa A, Panteleeva O

Moscow Helmholtz Research Institute of Eye Diseases, Moscow, Russian Federation

\section{Purpose}

To detect the ERG and VEP changes in severe and resistant forms of retinoblastoma (RB), and their dynamics after super selective intra-arterial chemotherapy (SIACT).

\section{Methods}

18 children (aged 11-48 months) were examined in two groups: (1) with RB resistant to earlier conducted treatment, including neoadjuvant polychemotherapy (NPCT) (14 eyes), and (2) with primarily diagnosed severe RB (8 eyes), whose first stage of treatment was SIACT. The flashVEP, standard cone ERG and flicker $30 \mathrm{HzERG}$, photopic negative response $(\mathrm{PhNR})$ in the red-on-blue ERG, photopic flicker ERGs (FERG) at 8.3, 10, 12, 24 and $30 \mathrm{~Hz}$ and mfERG were elicited with RETIport/scan21 (Roland Consult). MfERG (19 hexagons) was recorded before and 3 weeks after the SIACT in three eyes. Flash VEPs were tested in all patients before and after the SIACT, while the ERGs only in one eye with severe $\mathrm{RB}$ and in three eyes with resistant RB.

Results

For both forms of RB, the significant reduction of the cone ERG b-wave and standard flicker ERG up to $20-25 \%$ of the fellow eye values was revealed. The PhNR amplitude decrease was more significant in the eyes with resistant than severe RB (up to $12 \%$ and $29 \%$ respectively). FERG amplitude decreased most significantly for $24 \mathrm{~Hz}$ : up to $8 \%$ and $12 \%$ (of the fellow eyes) for the resistant and severe $\mathrm{RB}$ respectively, while the reduction of FERGs at 8.3-12 Hz was less prominent. This may reflect the greater dysfunction of bipolar cells than photoreceptors in RB. We found twice smaller amplitude and longer latency of N1components in ring 1 than in rings 2 and 3 of the mfERG. A similar tendency was found for the magnitude of P1 component. Three weeks after SIACT, an increase of 2.5-3 times was shown for $\mathrm{N} 1$ and $\mathrm{P} 1$ amplitudes in responses from the macula. Before treatment, the average amplitude of flashVEP P2in the group with resistant $\mathrm{RB}$ was $27.6 \%$ (of the fellow eyes), and in the group with severe RB was $75 \%$. Three weeks after the SIACT, the average VEP P2 amplitude in the resistant RB group increased up to $37.2 \%$ of fellow eye's values, and in severe RB eyes it was not changed or declined moderately.

\section{Conclusion}

In children with $\mathrm{RB}$, selective changes in the amplitudes of b-wave cone ERG, flicker ERG on $24 \mathrm{~Hz}, \mathrm{PhNR}$, and P1 of mfERG testified to the sharp dysfunction in bipolar and ganglion cells of the retina, more pronounced in the macula. After SIACT, macular function was improved more significantly compared to midperipheral retina function. In children with RB, the flash VEP may be a useful test in monitoring of chemotherapy, especially when the reliable registration of ERGs is not possible.

\section{PS A: Poster A6}

Recurrence of acute zonal occult outer retinopathy showing excellent response to steroid therapy

\section{Chai Yuzhu, Yamazaki H}

Department of Ophthalmology, Kohnodai Hospital, National Center for Global Health and Medicine, Chiba, Japan

\section{Purpose}

We present detailed ophthalmic findings in a case of acute zonal occult outer retinopathy (AZOOR) showing excellent response to steroid therapy at the second episode.

\section{Methods and results}

A 24 year-old woman visited our hospital for a sudden visual field defect with photopsia in the right eye. She had experienced the same symptoms at the age of 21 , and was diagnosed with AZOOR. Significant subjective and objective improvements were observed in the natural course at the first episode. At the second onset, her visual acuity was 0.8 in the right eye and 1.2 in the left eye. A relative afferent pupillary defect and an enlarged blind spot were found in the right eye. Mild inflammation in the anterior chamber and vitreous was 
observed in the right eye. Fluorescein angiography showed hyperfluorescence of the right optic disc. Pattern VEPs had a prolonged P100 latency of $140 \mathrm{~ms}$ when the right eye was stimulated. The amplitudes of both rod and cone full-field ERGs were reduced in the right eye. Disappearance of the inner segment/outer segment (IS/OS) line of the photoreceptors were observed by OCT in the right eye. Magnetic resonance imaging revealed no abnormalities. She was diagnosed with recurrence of AZOOR, possibly associated with optic neuritis. She was treated with steroid pulse therapy, and her visual acuity and visual field defect improved to normal in 2 weeks. At 1 month after the treatment, reappearance of the IS/OS line was detected by OCT. At 3 months, the P100 latency recovered to normal value and the reduced full-field ERGs recovered to normal size.

\section{Conclusion}

Our case showed that steroid therapy could be effective in treating AZOOR. Electrophysiological and OCT findings are helpful in diagnosis and following of patients with AZOOR.

\section{PS A: Poster A7}

PERG ratio correlation with Humphrey standard automatic perimetry and OCT in glaucoma and ocular hypertensive patients in a cross-sectional study in the Chilean population

\section{Cevo T, Hoehmann M, Metzler U}

Hospital Carlos van Buren, Universidad de Valparaiso, Chile

\section{Purpose}

Analyze the correlation between PERG ratio $\left(0.8^{\circ} / 16^{\circ}\right)$ and Humphrey standard automatic perimetry and OCT in ocular hypertensive (OHT) and glaucoma (GC) patients in the Chilean population.

\section{Methods}

16 OHT eyes and 23 GC eyes where studied through intraocular pressure curves, Humphrey standard automatic perimetry and OCT. Only patients with corrected visual acuity $>0.8$ were included. Patients had at least 3 previous coherent Humphrey standard automatic perimetry tests prior to entering the study. All patients had controlled ocular pressure through medical therapy or surgery prior to entering the study. Results

No statistically significant correlation was found between perimetry and PERG ratio for the OHT and the GC patients (Pearson correlation). A significant correlation was found between OCT RNFL thickness and PERG ratio in the OHT population ( $p=0.0154$, Pearson correlation). In the GC group correlation was found to be non-significant $(p=0.0521)$

\section{Conclusions}

PERG ratio has a significant correlation with RNFL in OHT patients in our analyzed group. Humphrey standard perimetry did not show a good correlation with either GC or OHT patients.

\section{PS A: Poster A8}

\section{Electrophysiological characterization of chick retinal function after neurotoxic damage using flash and pattern ERG}

\section{Fernandez-Nava D, Ostrin L, Wildsoet C}

University of California, Berkeley, CA, USA

\section{Purpose}

Several inner retinal cell populations have been implicated in the signal pathway that regulates eye growth and refractive error. To understand better the components of this pathway, neurotoxins have been used to ablate specific populations. Two such toxins are quisqualate, and colchicine, the former eliminating most amacrine cells, and the latter, peripheral glucagonergic amacrine cells, at least when used in low doses (Fischer 2008). Previous studies have primarily only examined retinal structural changes, and furthermore, usually through histology, which presents various confounds. More complete characterization of the effects of such neurotoxins, including changes over time, are now possible with in vivo modern imaging and functional recording systems. The purpose of this study is to investigate changes to retinal function in chicks exposed to the above neurotoxins.

\section{Methods}

Chicks (age 7d, $\mathrm{n}=30$ ) were monocularly injected with a single dose of $10 \mu \mathrm{l}$ of $250 \mathrm{ng}$ of colchicine (cch), $20 \mathrm{mM}$ quisqualate (QA), or phosphate buffered saline (PBS, control). Full field ERGs were recorded 3 and 7 days after cch and 7 days after QA or PBS injection. From the records, a-, b-, and c-waves, as well as photopic negative response (phNR) amplitudes were identified and treatment effects analyzed. A filter of $55-300 \mathrm{~Hz}$ was used to extract OPs from flash ERGs. ERGs were recorded in each eye 7 days after injection. PERG recordings included 13 increasing contrast levels (1-100 \%) for a $5^{\circ}$ alternating check size. 250 trials were collected for each step, and P50 and N95 amplitudes were analyzed. Induced retinal structural changes were tracked in vivo using spectral domain OCT (SD-OCT, Bioptigen, NC), from 4-10 days at 2 day intervals. The thicknesses of the individual retinal layers were computed and compared across treatment groups. For comparison, immunohistochemistry and TUNEL staining were used to determine the location of apoptotic cells.

\section{Results}

For full field ERG, cch injected birds exhibit an attenuation of a-wave $(p=0.002), \quad c$-wave $(p=0.002$,$) and phNR$ $(p=0.029$,$) , with no change in the b-wave (p=0.08$, $p=0.352)$. These decreases persisted 7 days after injection ( $p=0.037, p=0.048, p=0.007$ respectively). OPs in cch birds were not significantly affected. QA injected birds 7 days after injection exhibit an attenuation of a-wave $(p=0.018)$, b-wave $(p=0.024)$ and c-wave $(p=0.047)$ as well as a decrease in OP1 at around $18 \mathrm{~ms}(p=0.009)$ and OP2 at around $28 \mathrm{~ms}(p=0.002)$. For PERG, cch injected eyes demonstrated a decrease in the P50 amplitude $(p=0.000)$ for contrast levels greater than $10 \%$. TUNEL staining after cch confirmed cell death in the ganglion and inner nuclear layers, as well as some photoreceptor death. OCT imaging revealed significant thinning of the total retina, and ganglion cell, nerve fiber, and inner plexiform layers, significant 5-7 days after injections. 


\section{Conclusions}

The decrease of the PERG response, as well as the phNR of flash ERG, is consistent with structural changes in the inner retina observed using OCT and histology. ERG results also suggest that cch may also affect outer retinal function. In addition, the ERG suggests that the RPE is affected by cch and $\mathrm{QA}$, as indicated by decreases in the c-wave, which may be significant in terms of the role of the RPE as intermediary between retina and sclera in the pathway of eye growth regulation. The flash and pattern ERG both show promise as a sensitive measure of detecting inner and outer retinal changes to complement in vivo and ex vivo structural studies.

\section{PS A: Poster A9}

\section{Intravitreal injection of TTX induced two-phase photopic ERGs change in both Sprague-Dawley and Brown-Norway rats}

\section{Yang Hongfang, Wang Min, Sun Xinghuai}

Ophthalmology, Fudan University Affiliated Eye \& ENT Hospital, China

\section{Purpose}

To investigate short- and long-term effects of a simple intravitreal injection of TTX to retina of both albino rats and pigmented rats using photopic ERGs.

\section{Method}

Photopic ERGs were recorded on anesthetized adult SpragueDawley (4, male) and Brown-Norway rats (3, male) (ketamine/ xylazine, $50 \mathrm{mg} / \mathrm{kg}$ and $12.5 \mathrm{mg} / \mathrm{kg}$ ) using gold ring electrodes. ERGs were elicited using green Ganzfeld flashes (step1:11.38 cds $/ \mathrm{m}^{2}$, $0.333 \mathrm{~Hz}$; step2: $11.38 \mathrm{cds} / \mathrm{m}^{2}, 1 \mathrm{~Hz}$; step3: $22.76 \mathrm{cds} / \mathrm{m}^{2}, 0.333 \mathrm{~Hz}$; step4: $\left.22.76 \mathrm{cds} / \mathrm{m}^{2}, 1 \mathrm{~Hz}\right)$ and a green background $\left(40 \mathrm{~cd} / \mathrm{m}^{2}\right)$. Photopic negative response ( $\mathrm{phNR}$ ), $\mathrm{b}$-wave and $\mathrm{phNR}+\mathrm{b}$ were compared between TTX injected eyes and the contralateral sterile PBS injected eyes before and $30 \mathrm{~min}, 1 \mathrm{~h}, 2 \mathrm{~h}, 24 \mathrm{~h}$ and 1 week after a simple TTX intravitreal injection $(0.5 \mu \mathrm{mol} / \mathrm{l} 8 \mu \mathrm{l})$. HE stained paraffin sections were applied to evaluate the number of RGC and the thickness of inner nuclear layer after the last recording. All the evaluated indexes were calculated as TTX:PBS eye ratios.

\section{Results}

For SD rats, all the phNR, b- and $\mathrm{NR}+\mathrm{b}$ amplitude ratios (TTX:PBS) reduced to their lowest at $1 \mathrm{~h}$ after TTX injection before climbing almost to the pre-level at $24 \mathrm{~h}$ time point, but continued to decline in the following 1 week post injection: (1 h-24 h-1 week).PhNR: step1 0.662-1.047-0.828; step2 $0.676-1.225-0.623$; step3 $0.705-1.018-0.599$; step4 $0.711-$ $1.090-0.543$. b-wave: step1 0.979-1.044-0.629; step2 0.8941.249-0.466; step3 0.948-1.157-0.450; step4 0.709-1.097-0.368. PhNR + b: step10.726-1.032-0.703; step2 0.793-1.047-0.508; step3 0.750-0.985-0.509; step4 0.867-1.053-0.416. For BN rats, the results were similar except that trough appeared at $2 \mathrm{~h}$ post injection: (2 h-24 h-2 weeks). PhNR: step1 0.796-1.078-0.645; step2 0.806-0.913-0.678; step3 0.848-0.987-0.611; step4 0.8390.903-0.691. b-wave: step1 0.713-0.741-0.604; step2 0.718-0.7990.543; step3 0.736-0.763-0.578; step4 0.693-0.832-0.588. PhNR + b: step1 0.743-0.822-0.615; step2 0.747-0.821-0.615; step3 0.779$0.836-0.586$; step4 $0.740-0.855-0.609$. HE stained sections also showed that both RGC and cells in the inner nuclear layer decreased significantly 1-2 weeks after TTX intravitreal injection.

\section{Conclusion}

TTX intravitreal injection induced a two-phase decline of both phNR and b-wave in both albino and pigmented rats, which may reflect the electrical conductive function of RGC and bipolar cells in inner nuclear layer. The first phase is quick and reversible in several hours after injection, while the second is slow but irreversible in the following days to weeks.

\section{PS A: Poster A10}

Identification of a novel gene and novel mutations associated with LCA and juvenile RP

\section{Hui Wang}

Hangzhou Normal University, Hangzhou, China

\section{Purpose}

Leber congenital amaurosis (LCA) and juvenile retinitis pigmentosa (RP) are inherited retinal diseases that cause early-onset severe visual impairment. An accurate molecular diagnosis can refine the clinical diagnosis and allow gene-specific treatments. Methods

We developed a capture panel that enriches the exonic DNA of 163 known retinal disease genes. Using this panel, we performed targeted next-generation sequencing (NGS) for a large cohort of 179 unrelated and prescreened patients with the clinical diagnosis of LCA or juvenile RP. Systematic NGS data analysis, Sanger sequencing validation, and segregation analysis were utilized to identify the pathogenic mutations. Patients were re-visited to examine the potential phenotypic ambiguity at the time of initial diagnosis.

Results

Pathogenic mutations for 72 patients $(40 \%)$ were identified, including 45 novel mutations. Of these 72 patients, 58 carried mutations in known LCA or juvenile RP genes and exhibited corresponding phenotypes, while 14 carried mutations in retinal disease genes that were not consistent with their initial clinical diagnosis. We re-visited patients in the latter case and found that biallelic mutations of PRPH2 may cause LCA/ juvenile RP. Guided by the molecular diagnosis, we reclassified the clinical diagnosis in 2 patients.

\section{Conclusions}

We have identified a novel gene and a large number of novel mutations that are associated with LCA and juvenile RP. Our results highlight the importance of molecular diagnosis as an integral part of clinical diagnosis.

\section{PS A: Poster A11}

Comparison of normal SD rat ERG by two visual electrophysiology systems: GT2000NV and RETIscan under different color stimuli

\section{Cao Qinglin, Cao Qing-Lin, Zhang Zuo-Ming, An Jing, Zhang Lei}

School of Aerospace Medicine, Fourth Military Medical University, $\mathrm{Xi}^{\prime}$ an, China 


\section{Purpose}

To compare the ERGs of normal SD rat recorded by two visual electrophysiology systems: GT2000NV and RETIscan and to evaluate the features of rat ERG under different color stimuli. Methods

Using the GT-2000 and RETIscan systems, six rats were stimulated by red, blue, green and white light respectively after dark adaptation and the ERG was recorded. The parameters of two systems were identical or close.

\section{Results}

Excluding the amplitude of the white light cone-ERG b waves, the other ERG values were significantly different between the two systems. The ERG responses under blue and green light stimulus were stronger compare with the responses under red and white light stimulus excepting the OPs, but there were no significant differences.

\section{Conclusions}

Most of the results between the two systems have significant differences, which are mainly caused by the system-specificity. As the retinal photoreceptors of rat are mainly composed of rods, which are sensitive to about $500 \mathrm{~nm}$ wavelength, and its cone system lacks L-cones, we found that most of the ERGs were stronger under blue and green light stimulus than red and white. OPs reflects the retinal neurons electrical activity; it maybe more complex and further work should be done to formulate the mechanism of rat ERG under different color stimuli.

\section{PS A: Poster A12}

\section{A mutation in GUCA1A gene causes cone dystrophy in a Chinese family}

\section{Shijie Zheng, Hui Wang, Rui Chen, Bo Lei}

Department of Ophthalmology, the First Affiliated Hospital of Chongqing Medical University, Chongqing Key Laboratory of Ophthalmology, Chongqing Eye Institute, Chongqing, China

\section{Purpose}

To investigate the underlying molecular genetic basis of cone dystrophy identified in a Chinese family.

\section{Methods}

The proband was a 21-year-old female college student who complained of progressive decrease of central vision, poor visual acuity in daylight, photophobia and dyschromatopsia for 6 years. Detailed ophthalmologic examinations, color fundus photography, macular pigment density, OCT and dark- and light-adapted ERG assessments were performed. Blood samples were taken for DNA extraction, DNA sequencing was performed followed by Sanger validation.

\section{Results}

Best corrected visual acuity (BCVA) was 20/200 in both eyes. Foveal reflex was absent and macular pigment density significantly reduced. OCT showed the thickness of the retina especially the outer nuclear layer in the macular area was reduced, and the photoreceptor inner segment/outer segment (IS/OS) junction was disrupted and missing. ERG revealed profoundly decreased and prolonged light-adapted ERG and flicker responses, but preserved dark-adapted ERGs. Direct sequencing and mutational analysis revealed an $\mathrm{A}$ to $\mathrm{T}$ transition in exon 4 of GUCA1A gene which encodes the guanylate cyclase-activating protein 1 (GCAP1), resulting in a glutamic acid to valine (E111 V) in GCAP1 in the proband. The mutation was not present in her parents or other healthy controls.

\section{Conclusions}

A novel c.A332T (p.E111 V) mutation in gene GUCA1A was identified in a Chinese family with cone dystrophy and the patient shared similar clinical and ERG characteristics with other autosomal dominant cone dystrophy caused by mutations in GUCA1A. Previous reports have identified 10 mutations in the GUCA1A gene in Caucasian families with cone dystrophy. Our data confirmed that GUCA1A gene mutations also cause similar cone system dysfunction in Chinese population.

\section{PS A: Poster A13}

No need to avoid high luminances in the mfERG tobetterbring out the blind spot

\section{Poloschek C, Bach M}

Department of Ophthalmology, University of Freiburg, Freiburg, Germany

\section{Purpose}

The optic nerve head (ONH) has higher reflectance than neighboring retinal tissue; this could explain why it is frequently not picked up in the mfERG; non-linearities may also play a role. We here ask whether detection of the $\mathrm{ONH}$ depends on stimulus luminance.

\section{Methods}

We obtained four mfERG recordings from 11 eyes (6 subjects, $26 \pm 3 \mathrm{yr}$ old), two with natural pupils and two in mydriasis, with a luminance of $150 \mathrm{~cd} / \mathrm{m}^{2}$ and $500 \mathrm{~cd} / \mathrm{m}^{2}$, respectively, resulting in four different retinal illuminances ("low", "medium", "high", "very high") spanning a factor of 18. Stimulus resolution was 61 scaled hexagons per eye. In right eyes the hexagon that most covered the optic disc was the second to the right in the horizontal meridian; in left eyes it was the second to the left in the horizontal meridian. Amplitude ratios from these two hexagons were calculated.

\section{Results}

There is a slight tendency for a "less deep" ONH response for higher illuminances, but there is no significant correlation between the four different retinal illuminances and amplitude ratios of the blind spot. However, the scatter of amplitude ratios at the "low" illuminance condition is twice as large compared to that at the "high" condition, because in the "high" condition amplitude is higher and consequently the peaks are better defined.

\section{Conclusions}

Avoiding high retinal illuminance in order to improve the detectability of the blind spot seems not effective. On the contrary, using high retinal illuminances shows equally good results and has the advantage of higher local amplitudes, making it easier to assess local pathology manifesting as localized amplitude loss. 


\section{PS A: Poster A14}

Effects of repeated intravitreal bevacizumab injections on the inner-retinal function in neovascular age-related macular degeneration

Kim Hoon Dong, Seung Hoon Kim, In Hwan Cho, Chan Hee Moon, Tae Kwann Park, Young-Hoon Ohn

Department of Ophthalmology, College of Medicine, Soonchunhyang University, Bucheon, Republic of Korea

\section{Purpose}

To evaluate the effects of repeated intravitreal bevacizumab injections on the retinal function in eyes with neovascular agerelated macular degeneration (AMD).

\section{Methods}

Full-field ERGs (ffERGs) and photopic negative responses $(\mathrm{PhNR})$ were recorded in 30 eyes of 30 patients with neovascular AMD. All the participants were treated with three-time six-week scheduled intravitreal bevacizumab injections. Comprehensive ophthalmic examinations including electrophysiological studies were performed before the treatment (baseline), four weeks after each intravitreal bevacizumab injection, and six months after the first injection. The amplitude of PhNR was defined as the difference between baseline and the lowest peak of the negative wave following the cone b-wave. Results

None of the standard ffERG parameters changed significantly 6 months after the repeated intravitreal bevacizumab injections. PhNR amplitude and $\mathrm{PhNR} / \mathrm{b}$-wave ratio decreased four weeks after the first injection but with no significant change ( $p=0.13, p=0.49$, respectively). However, four weeks after the second injection, $\mathrm{PhNR}$ amplitude and $\mathrm{PhNR} / \mathrm{b}$-wave ratio significantly decreased $(p<0.001, p=0.010$, by paired t-test with Bonferroni correction), and reached the minimum value compared to baseline. They recovered gradually and showed no significant difference from baseline $(p=0.39, p=0.45)$ at endpoint.

\section{Conclusions}

The results suggest that repeated intravitreal bevacizumab injections might induce temporary dysfunction of the inner retina, but showed no significant toxicity to the photoreceptor function. As the dysfunction of the inner retina was a reversible change, repeated treatment may not be a serious consideration when using intravitreal bevacizumab for the treatment of AMD.

\section{PS A: Poster A15}

Treatment for limited long-standing retinal detachment with Stargardt disease

\section{Xiaoyong Huang, Ya Qu}

Southwest Eye Hospital, Southwest Hospital, Chongqing, China

A 38-year-old man was admitted to our hospital with a 20 -year history of visual deterioration of left eye. There was no history of ocular injury. Visual acuity (OD) was $8 / 20$, and could be corrected to 10/20; visual acuity (OS)was $2 / 20$ and could not be corrected. In the left eye, binocular indirect ophthalmoscopy revealed a horizontal oval of atrophic pigment epithelium appears, which measures about 2.0 disc diameters in width and 1.5 disc diameters in height; a broad ring of flecks surrounds this area. A shallow localized detachment is present nasal to the optic disconly and there is a tide-mark indicating chronicity, but no retinal hole was found through careful examination with three-mirror contact lens. Intraocular pressure of right eye was $13 \mathrm{mmHg}$, intraocular pressure of left eye was $10 \mathrm{mmHg}$. Serum glucose was normal. Fluorescein angiogram showed many flecks: the entire posterior pole show a blotchy pattern of hyperfluorescence and central choroidal atrophy was visible. Ultrasound B-scan showed localized retinal detachment of left eye. MfERGs showed central amplitudes decreased significantly. OCT showed no retinal detachment in fovea. Diagnosis include: retinal detachment and Stargardt disease of the left eye, reflective error of both eye. Because of the difficulties of treating his left eye and poor prognosis, the patient is presented for discussion of further management, specifically whether to be treated with laser demarcation to reduce the risk of progression, or to undertake other retinal reattachment surgery. The aim is to achieve best results in managing a patient with a condition with such low success rate and such dim outcome.

\section{Poster Session B}

\section{PS B: Poster B1}

Observation of the clinical efficacy of perceptual learning training in the treatment of elder amblyopia patients

\section{LinBo He, Bo Liu, Tao Yu, Hong Yang, Hui Wang}

Southwest Hospital, Southwest Eye Hospital, Third Military Medical University, Chongqing, China

\section{Purpose}

To observe the clinical efficacy of perceptual learning training in the treatment of older patients with amblyopia.

\section{Methods}

Prospective case study where 43 amblyopic subjects undertook the perceptual learning training. Before training, $\log M A R$ visual acuities were taken for the non-amblyopic eye and the amblyopic eye with best correction. Seven sets of spatial frequency contrast sensitivity function (CSF) measurements were also taken. With the nonamblyopic eye covered, perceptual learning training was performed on the amblyopic eye for $30 \mathrm{~min}$ once a day, for a treatment course of 10 days. At the end of the 10 day treatment period, logMAR visual acuities and contrast sensitivity function measurements were measured for the non-amblyopic and amblyopic eye.

\section{Results}

Before treatment, the mean $\log$ MAR visual acuity of the nonamblyopic eye was $0.033 \pm 0.112$. After treatment, the mean $\log$ MAR visual acuity was $0.016 \pm 0.107$. This was a statistically significant change $(p<0.05)$. Before training, the average $\log$ MAR visual acuity of the amblyopic eye was $0.487 \pm 0.358$ while after training, the mean $\log$ MAR visual acuity was $0.406 \pm 0.286$; a statistically significant change $(p<0.01)$. For all 7 sets of spatial frequencies, the changes in contrast sensitivity 
for the non-amblyopic eye were not statistically significant. For the amblyopic eye, comparing the contrast sensitivity of spatial frequencies $0.5,1$ and 2 taken before and after training, no statistically significant improvements were noted. However, for $4,8,12$ and 16 , there were statistically significant improvements. For amblyopic patients $\leq 12$ years old, the changes in contrast sensitivity before and after training, at all 7 spatial frequencies, were not statistically significant in both the non-amblyopic and amblyopic eye. For amblyopic patients $>12$ years old, the difference in contrast sensitivity before and after training for the non-amblyopic eye was statistically significant only at the spatial frequency of 16 and not for the other 6 groups. In comparison, the difference in contrast sensitivity before and after training for the amblyopic eye was statistically significant for the spatial frequencies of $0.5,1,2,8,12$ and 16 .

\section{Conclusion}

Perceptual learning can significantly improve visual acuity and contrast sensitivity in amblyopic patients with some improvements seen in the non-amblyopic eye. This type of training displayed better results for the amblyopic eye in older subjects compared to younger subjects.

\section{PS B: Poster B2}

\section{Natural history of five patients with acute zonal occult outer retinopathy}

Tanaka $\mathbf{H}^{1,2}$, Fujinami $K^{1,4}$, Noda $T^{1,2}$, Miyake $Y^{1,5}$, Tsunoda $\mathbf{K}^{1,2}$

\author{
${ }^{1}$ National Institute of Sensory Organs, Tokyo, Japan, \\ ${ }^{2}$ National Hospital Organization, Tokyo Medical Center, \\ Tokyo, Japan, ${ }^{3}$ Keio University, School of Medicine, \\ Tokyo, Japan, ${ }^{4}$ UCL Institute of Ophthalmology, London, \\ UK, ${ }^{5}$ Aichi Medical University, Aich, Japan
}

\section{Purpose}

To describe the natural history of patients with acute zonal occult outer retinopathy (AZOOR).

\section{Methods}

Five patients diagnosed as AZOOR and followed over two years were ascertained. The clinical diagnosis of AZOOR was made based on the characteristic features described by Gass (Gass et al. 1992; Gass et al. 2002); acute onset of zonal field loss associated with photopsia, unremarkable fundus changes, and ERG abnormalities. A detailed history and a comprehensive ophthalmic examination was undertaken, including visual acuity (VA), visual field (VF) test, fundus imaging, and spectral-domain OCT (SD-OCT). Electrophysiological assessment was performed with ISCEV standard full-field ERGs (ffERGs) and mfERG/focal macular ERG (FMERG). The data obtained at baseline were compared to those at follow-up.

\section{Results}

Five patients were recruited (four female and one male). The mean age of onset was $33.8 \mathrm{yr}$ (range 23-44 yr) and the mean follow-up interval was $4.8 \mathrm{yr}$ (range $2-7$ years). Initial complaint of four patients was unilateral visual loss and two subjects among them showed an asymptomatic retinal disorder in the other eye; one individual noticed bilateral blind spot enlargement. Two patients with central scotoma detected by VF test had severely decreased VA (group 1); the VA was preserved both in two subjects with bilateral ring scotoma connected to enlarged blind spot (group 2) and one individual with para-central scotoma (group 3). VA was maintained in all patients over the follow-up period, but the enlargement of VF defect was observed in two patients (group 2). SD-OCT detected disappearance of the focal-spotted lesion in the outer retinal layers in one patient (group 3). ffERGs demonstrated abnormal responses in four patients (group 1 and 2); one subject (group 1) showed improvement of cone responses. MfERG/FMERG detected the enlarged area with reduced responses in one patient (group 3).

\section{Conclusion}

The variable natural history of patients with AZOOR was described. Progression of the disease was observed in group 2; meanwhile improvement was demonstrated in two patients (group 1 and 3). The type of VF defect at the initial visit may indicate the prognosis of AZOOR.

\section{PS B: Poster B3}

\section{Binocular asymmetry of retinitis pigmentosa patients:clinical phenotype analysis}

\section{Hongxuan Lie, Shiying Li, Gang Wang, Ming Wang, Mingfang Zhang, Sun, Zhengqin Yin}

Southwest Hospital/Southwest Eye Hospital, Third Military Medical University, Chongqing, China

\section{Purpose}

Analyse binocular asymmetry of primary retinitis pigmentosa (RP) patients' clinical phenotypeto explore the clinical characteristics of different processes in two eyes of the same individual patient and possible prevention mechanisms.

\section{Methods}

A retrospective analysis of our hospital clinical data from 2005 to 2013 revealed 1,450 cases of adult patients diagnosed with RP. Patients with differences caused by asymmetrical lesions (such as asymmetric cataract, refractive errors) were excluded through visual acuity and fundus autofluorescence imaging (FAF) inspection. Screening out patients with the diameter of hyperautofluorescent ring differences on the same individual eyes $>5 \mathrm{~mm}$, the visual acuity differences on the same individual eyes $>20$ letters (Early Treatment Diabetic Retinopathy Study ETDRS visual chart). The patients who are screened out to examine FAF and OCT to detect the morphology of retina, then to examine mfERG, flash ERG and visual field (VF). To detect possible protectors of slow down the progressive disease, we examine the thickness of the choroid and armto-retinal circulation time, axis oculi and intraocular pressure.

\section{Results}

Binocular asymmetric progression of retinal lesions were rare in RP patients $(1 \%, 11 / 1,450)$. The clinical presentation is characterized by: the eye with poorer visual function displaysa hyperautofluorescent ring diameter smaller than the other eye and closer to the fovea.OCT shows disappearance of the IS/ OS layer and the external membrane location closer to the fovea in the eye with poorer visual function, the area just disappear corresponding on the hyperautofluorescent ring 
boundaries. FERG and mfERG amplitude reduction and latencies extension is more obvious in the eye with poorer visual function.

\section{Conclusion}

Patients with large differences in RP progression between eyes are rare, and its progression in the death of photoreceptor cells in the retina associated with Muller outer endplate lost. Binocular asymmetric of primary RP possible mechanisms of the process to be further explored.

\section{PS B: Poster B4}

\section{Generalized retinal dysfunction in a case of early stage macular telangiectasia type 2}

\section{Ledolter AA, Hasler PW, Palmowski-Wolfe AM}

Department of Ophthalmology, University of Basel, Basel, Switzerland

\section{Purpose}

Macular telangiectasia type 2 is characterized by bilateral dilatation and tortuosity of juxtafoveolar capillaries. Its aetiology is unknown. In individual patients, retinal dysfunction has been discussed as a possible underlying pathology. Here we describe retinal function in another patient with early stage of macular telangiectasia type 2 .

\section{Methods}

A 43-year-old woman complained of blurred vision, predominantly in the left eye. Three months earlier, restless-legssyndrome (RLS) had been diagnosed and benzodiazepine was prescribed. The patient underwent detailed ophthalmic clinical evaluations including: static automated perimetry (SAP) (Octopus 900, Haag-Streit, Switzerland), fundus autofluorescence (FAF), fluorescein angiography (FA) (Spectralis HRA2, Heidelberg, Germany); OCT (Cirrus HD-OCT, Carl Zeiss, Meditec, Switzerland); EOG and full-field ERG (Roland Consult, Germany); mfERG (VERIS by EDI, San Mateo, California, USA). Results

Best corrected Snellen acuity was $1.2 \mathrm{OD}$ and 1.0 OS. Both eyes were myopic (OD $-6.0 \mathrm{D} /-2.0 / 7^{\circ}$ and $\mathrm{OS}-6.25 \mathrm{D} /-1.25 /$ $165^{\circ}$ ). No relative afferent pupillary defect was observed. Orthoptic examination was normal with good motility and stereo functions. Slit lamp examination showed no abnormality OU. Mild pigment irregularities were noted in the macula on fundus examination. SAP revealed diffuse lowering of sensitivity in the lower range of normal in her right eye and a local visual field defect superior-temporal to the blind spot on the left eye. FAF showed perifoveal punctually decreased AF OU. FA revealed a subtle diffuse hyperfluorescence temporal to the fovea OD $>$ OS. The OCT revealed a thinning of the retina in the temporal macula of both eyes. EOG was normal (Arden ratio: $2.2 \mathrm{OD}, 1.8 \mathrm{OS}$ ). Photopic ERG showed reduced amplitudes with increased latencies; scotopic ERG revealed reduced amplitudes with normal latencies. Three months after stopping benzodiazepine therapy, scotopic ERG responses were still reduced, while photopic ERG responses were in the lower range of normal. A retinopathy was postulated. MfERG responses were in normal range compared to control except for single traces temporal OS $>$ OD.

\section{Conclusion}

Based on the typical fluorescein angiographic changes, early macular telangiectasia type 2 was diagnosed. Histological analysis of an eye from a patient with macular telangiectasia type 2 revealed prominent loss of Müller cell markers in the central retina. This suggests an important role of Müller cells in pathogenesis of the disease. Müller cell ablation leads to photoreceptor apoptosis and vascular telangiectasis, bloodretinal barrier breakdown and may lead later to intraretinal neovascularization. In a transgenic mouse-model for conditional Müller cells ablation, overnight dark adapted ERG shows both dysfunctional rod- and cone-derived b-waves. In humans, reduction of amplitudes of cone-and rod-driven responses have rarely been reported in telangiectasia. Our findings of reduced b-wave amplitudes in a patient with early macular telangiectasia type 2 support the theory that telangiectasia is a more generalized retinal disorder than previously appreciated. ERG examination in these patients may help to explain unclear visual abnormalities and can help estimate retinal function.

\section{PS B: Poster B5}

\section{Clinical usefulness of binocular mfERG in patients} with monocular macular disease

\section{Kyung Seek Choi, Hoon Dong Kim, Hyun Joon Lee, Young Hoon Ohn}

Soonchunhyang University Seoul Hospital; Soonchunhyang University Bucheon Hospital, Seoul, Republic of Korea

\section{Purpose}

To evaluate the clinical usefulness of binocular mfERG by comparing results with conventional monocular mfERG in patients with monocular macular disease.

\section{Methods}

MfERG testing was conducted on 32 patients with monocular macular disease and 30 normal subjects. An initial mfERG was recorded from both eyes simultaneously with 2 recording electrodes under binocular stimulation. A second mfERG was subsequently recorded with conventional monocular stimulation. Amplitudes and implicit times of each ring response of the binocular and monocular recordings were compared. Ring ratios of the binocular and monocular recording were also compared.

Results

In the macular disease group, there were no statistical differences in amplitudes and implicit times for each of the five concentric rings between the monocular and binocular recording, whereas with binocular simulation, the ring ratios (ring1/ring4, ring1/ring5) were significantly reduced in the affected eye. In the normal control group, there were no statistical differences in all parameters between the monocular and binocular recording.

\section{Conclusions}

The binocular test can be considered for patients who cannot perform monocular fixation due to macular disease. In addition, given the stable fixation of the affected eyes during the binocular test, the reliability of the test results could be improved. 


\section{PS B: Poster B6}

Analysis of a-wave components in the maximal combined rod-cone response of flash ERGs in Stargardt disease and cone dystrophy

Gang Wang, Shiying Li, Min Wang, Jiaman Dai, Xiaohong Meng, Zhengqin Yin

Southwest Hospital/Southwest Eye Hospital, Third Military Medical University, Chongqing, China

\section{Purpose}

In the dark-adapted 3.0ERG, formerly called the maximal combined rod-cone ERG, the a-wave has double troughs. Some scholars consider the first trough to be caused by a cone response and the second by a rod response, while other scholars consider the formation of double troughs a result of the interruption of OP1. This study aims to find some evidence for the origin of the a-wave.

\section{Method}

To analyse the a-wave of the maximal combined rod-cone ERG in 10 cases with Stargardt disease and 10 with cone dystrophy.

\section{Results}

In cases with Stargardt disease and cone dystrophy, OP1 is obvious, and the a-wave of the light-adapted 3.0 ERG (formerly the single-flash cone ERG) decreased severely. The a1-wave of the maximal combined rod-cone ERG is not obvious while the a2-wave is obvious. When the flash stimulus was increased to $50 \mathrm{cdsm}^{-2}$, the a2-wave increased significantly in amplitude while the a1-wave didnot.

\section{Conclusion}

In the maximal combined rod-cone ERG, the a-wave is not affected by the OPs. The a1-wave, the first trough of the a-wave, is caused by the cone response.

\section{PS B: Poster B7}

MfERG and OP amplitudes correlate with severity of diabetic maculopathy better than best corrected visual acuity (BCVA)

\section{Brown M, Raj A, Sahni J, Campa C, Czanner G, Hagan R, Denby C, Broadbent D, Harding S, Fisher A}

Department of Medical Physics and Clinical Engineering, Royal Liverpool University Hospital \& University of Liverpool, Liverpool, UK

\section{Purpose}

To determine objective and subjective macular function in the presence of varying levels of diabetic maculopathy.

\section{Methods}

Treatment naïve patients with diabetes mellitus (DM) were recruited prospectively into four groups: (1) DM but no retinopathy; (2) early diabetic maculopathy but no features of clinically significant macular edema (CSME); (3) CSME; (4) ischemic maculopathy. A group of healthy volunteers without DM were also recruited. All subjects underwent BCVA, contrast sensitivity (CS), fluorescein angiography (FA), mfERG, OP, microperimetry (MP) and assessment for systemic risk factors. Statistical analysis was performed on SPSS version 20 using ANOVA; post hoc analysis was performed using the Sidak test.

Results

No statistically significant difference was found in BCVA or CS between controls and subjects in groups 1-3; however, subjects with ischemic maculopathy showed significantly reduced BCVA and CS $(p<0.02)$. Subjects with early maculopathy, CSME and ischemic maculopathy showed a significantly reduced mfERG central ring amplitude ( $p=0.014, p<0.001$ and $p<0.001$ respectively) as compared to healthy controls. MP central ring sensitivity was similarly reduced in these three groups compared to healthy controls ( $p<0.05, p=0.001$ and $p<0.001$, respectively). OP sum amplitude was significantly reduced in subjects with CSME $(p<0.01)$ and ischemic maculopathy $(p<0.001)$ as compared to healthy controls and subjects in group 1 and 2 . MfERG central ring implicit time and OP 1st peak implicit time were prolonged with increasing severity of disease though statistical significance was not reached.

\section{Conclusions}

Neural macular function appears to decrease with increasing severity of disease, and especially in the presence of macular ischemia. MfERG, MP and OP appear to be more sensitive markers of macular function than visual acuity or contrast sensitivity

\section{PS B: Poster B8}

Clinical study of Stargardt disease

Hui Li Ruifang, Sui Huajin Li, Ruxin Jiang

Department of Ophthalmology, Peking Union Medical College Hospital, Chinese Academy of Medical Sciences, Beijing, China

\section{Purpose}

To study the clinical features of Chinese patients with Stargardt disease related to ABCA4 mutations.

\section{Methods}

Seventeen unrelated patients diagnosed with Stargardt disease and ABCA4 mutations. Seven patients were male and twelve patients were female. The patients' ages ranged from 8 to 44 years. All patients underwent detailed ocular examinations including ERG, OCT, visual field and fundus antofluorescence (FAF).

\section{Results}

All patients showed impaired visual acuity except one adult patient who had normal visual acuity with fundus flavimaculatus. 7 patients with visual field data have progressive loss of central vision and near normal peripheral visual fields. 16 patients with OCT images show disorganization and/or loss of the IS-OS junction in the foveal area. Full-field ERG showed that 3 patients were normal; 11 patients had both rod and cone dysfunction and 3 patients had isolated cone dysfunction. Common FAF features include well-defined low AF signal in macular region surrounded by high AF flecks; low AF signal patches and high AF signal flecks were observed in the posterior pole and midperipheral retina. 


\section{Conclusions}

Clinical heterogeneity exists in Stargardt disease patients related to ABCA4 mutations. FERG maybe used to classify the type of photoreceptor dysfunction present for these patients (normal, cone dysfunction and rod and cone dysfunction). Patient with fundus flavimaculatus may have more visual function preserved.

\section{PS B: Poster B9}

\section{Clinical characteristics of paraneoplastic retinopathy and optic neuropathy}

HouBin Huang, Shi-hui Wei, Ying Li, Feng-xiang Wang, Yi Yao, Cai-hui Jiang, Zheng-qin Yin, Mao-nian Zhang, Wen-bin Wei

Department of Ophthalmology, the General Hospital of Chinese People Liberation Army, Beijing, China

\section{Purpose}

To analyze the clinical characteristics of paraneoplastic retinopathy and optic neuropathy (PRON).

\section{Methods}

Case series study. Nine patients were enrolled from October 2006 to March 2013 who visited the ophthalmology department of the PLA General Hospital. The patients were underwent a series of examinations, including fundus photography, visual electrophysiology, fundus fluorescein angiography, OCT, fundus autofluorescent imaging, perimetry, ultrasonography, magnetic resonance imaging, spinal tap and cerebrospinal fluid test, paraneoplastic syndrome (PNS) antibody test. The patients were followed up in outpatient department and/or by phone. The clinical manifestation, entity types, and treatment were analyzed. Results

Of the nine patients, there were 3 cases of cancer associated retinopathy (CAR), 3 cases of bilateral diffuse uveal melanocytic proliferation (BDUMP) and 3 cases of paraneoplastic optic neuropathy (PON). In five patients PNS antibodies were detected and three patients were revealed with positive results. As for the primary malignancy, four of the nine patients were lung carcinoma, others included invasive thymoma, kidney cancer, carcinoma of gastric cardia, acute lymphocytic leukemia and cervical cancer, each for one case. All the patients complained of vision blurring or progressive visual decrease. Other complaints included dark shadow in two patients, shimmering, dazzling, double vision and eye pain, each in one patient. One patient complained of progressive decreased vision in both eyes prior to the diagnosis of lung cancer. Of the 18 eyes of the nine patients, there were six patients with no light perception, five with vision from light perception to 0.05 , and other five with 0.4 vision or betterat the end of follow up. Five patients were treated with steroids with unsatisfactory efficacy.

\section{Conclusions}

Each entity of PRON has its own clinical characteristics. PRON especially BDUMP, may be a pre-metastatic disease. It is important for practicing clinicians especially for ophthalmologist or neuro- ophthalmologist to be familiar with each syndrome to allow early diagnosis of the disease and the underlying malignancy.

\section{PS B: Poster B10}

\section{Autoantibodies against TRPM1 in Japanese patient} with melanoma-associated retinopathy

Kondo $\mathbf{M}^{1}$, Morita $\mathbf{Y}^{2}$, Kimura $\mathbf{K}^{2}$, Fujitsu $\mathbf{Y}^{2}$, Enomoto $A^{3}$, Ueno $S^{3}$, Sonoda $K^{2}$

${ }^{1}$ Department of Ophthalmology, Mie University Graduate School of Medicine, Japan, ${ }^{2}$ Yamaguchi University Graduate School of Medicine, Japan, ${ }^{3}$ Nagoya University Graduate School of Medicine, Japan

\section{Purpose}

To report our findings in a Japanese patient with melanoma associated retinopathy (MAR) who was found to have autoantibodies against the transient receptor potential cation channel, subfamily M, member 1 (TRPM1).

\section{Methods and results}

Our patient complained of blurred vision, night blindness, and photopsia. Fundus photographs, fluorescein angiograms, and the spectral domain OCT (SD-OCT) findings were essentially normal. However, the full-field scotopic ERGs had a negativetype pattern in both eyes suggesting extensive bipolar cell dysfunction. Systemic examination revealed that our patient had malignant melanoma of the lungs and anus. Autoantibodies against TRPM1 were identified in the serum of this patient by Western blot analysis. After oral corticosteroid therapy, his visual symptoms and vitreous opacity were markedly improved. The patient died from the complications of the melanoma.

\section{Conclusion}

Our case is the first MAR patient in whom the TRPM1 autoantibody was detected in an ethnic group other than the Caucasians.

\section{PS B: Poster B11}

Functional and structural changes after pattern scanning laser photocoagulation in diabetic retinopathy

\section{Zhongshan Chen, Yanping Song}

Department of Ophthalmology, Wuhan General Hospital of Guangzhou Military Command, Wuhan, China

\section{Purpose}

To compare retinal function and morphology of diabetic retinopathy after treatment by pattern scanning laser photocoagulation and conventional multi-wavelength laser photocoagulation.

\section{Methods}

160 patients diagnosed with proliferative diabetic retinopathy were randomly divided into group A: pattern scanning laser photocoagulation group and Group B: multi-wavelength laser group. All the patients underwent panretinal photocoagulation. Before and after treatment of 1 week, 1 month, 6 months and 12 months the patients underwent fundus photography, OCT, microperimetry and fundus autofluorescence (AF).

Results

At the same time point after treatment, fundus photography showed lighter photocoagulation spots in group A than group B. The range of light spots was more limited in group A, and 
became more diffused in group B and appeared more proliferative pigment at late stage. OCT showed the lesions limited in RPE layer in group A, but diffused both in RPE and inner neural retina in group B. Microperimetry indicated higher light sensitivity in the macula of group A than that of group B. Lesser AF changes appeared in group A than in group B, and the lesion range were enlarged or $\mathrm{AF}$ was discontinuous.

\section{Conclusions}

The pattern scanning laser photocoagulation is a safe, rapid, effective method for diabetic retinopathy. Pattern scanning laser photocoagulation can benefit from smaller inner retina damage, more preservation of retinal fine function, better protection for retinal barrier than conventional laser photocoagulation.

\section{PS B: Poster B12}

Clinical study of the therapeutic effect of conjunctival flap cover surgery done on corneal perforation due to infective corneal ulcer

\section{Sha Zheng, Hanping Xie, Yuli Yang, Chunming Hu, Jie Xiong}

Southwest Eye Hospital, Southwest Hospital, the third Military Medical University, Chongqing, China

\section{Purpose}

To investigate the therapeutic effect of conjunctival flap cover surgery done on corneal perforation due to infective corneal ulcer Methods

Conjunctival flap cover surgery was performed on 95 patients (95 eyes) admitted to our hospital suffering corneal perforation due to infective corneal ulcer. Male/female ratio 3:1, age ranged from 35 to $76 \mathrm{yr}$ (average $53 \mathrm{yr}$ ), follow-up period after surgery ranged from 7 to 20 months. Infection types: viral 37 eyes, fungal 49 eyes, bacterial 9 eyes.

\section{Results}

95 eye balls were all preserved after surgery. 3 months to 6 months after conjunctival flap cover surgery, conjunctival flap removal surgery was done on 27 eyes. After conjunctival flap removal surgery, those patients' corneas were smooth and transparent without neovessels, and improvements in different degrees were obtained in their vision acuity (the best visual acuity was 0.2 ).

\section{Conclusions}

Conjunctival flap cover surgery is applicable for infective corneal perforation in which diameter is less than $2 \mathrm{~mm}$. For this kind of infective corneal perforation, conjunctival flap cover surgery works effectively to save the eyeball and supplies a base for the next, vision-improving surgery.

\section{PS B: Poster B13}

The test-retest reliability of the photopic negative response (phNR)

Tang $J^{1}$, Edwards $T^{2}$, Crowston $J^{1,2}$, Sarossy $M^{1,2}$

${ }^{1}$ Glaucoma Research Unit, Centre for Eye Research Australia (CERA), Melbourne, Australia, ${ }^{2}$ The Royal Victorian Eye and Ear Hospital, Melbourne, Australia

\section{Purpose}

The photopic negative response (PhNR) has been suggested as an early indicator of glaucomatous damage. There is limited evidence concerning the reliability of the common approaches to measure its amplitude. This study seeks to assess the testretest reliability of the $\mathrm{PhNR}$ by these different approaches and to compare this reliability to that of the b-wave of the ERG.

Methods

Photopic ERGs were recorded from the right eye of 31 normal subjects (mean age $=34.4 \mathrm{yr}$, range $=21-72 \mathrm{yr}$ ) on two occasions separated by mean 7.5 days. Signals were acquired using DTL electrodes in response to brief red Ganzfeld flashes $\left(2.25 \mathrm{cds} / \mathrm{m}^{2}\right)$ on a rod suppressing blue background $\left(10 \mathrm{~cd} / \mathrm{m}^{2}\right)$. b-wave amplitude was measured from a-wave trough to peak. PhNR amplitude was recorded from pre-stimulus baseline to PhNR trough (BT), b-wave peak to PhNR trough (PT) and b-wave peak to mean implicit time (PF); a ratio of b-wave/peak-to-trough (PTR) was also evaluated. Reliability was assessed using intra-class correlation coefficient (ICC2,1) and inter-session limits of agreement (LOA).

Results

ICC $(95 \%$ CI) for b-wave was $0.66(0.40-0.82)$ with LOA of $53 \%$. ICC for BT, PT, PF amplitudes and PTR were 0.30 (0-0.59), 0.59 (0.31-0.78), $0.54(0.24-0.75)$ and $0.25(0-0.55)$, respectively. LOA for PhNR measurements were $153 \%, 51 \%$, $52 \%$ and $37 \%$.

\section{Conclusions}

The peak-to-trough $\mathrm{PhNR}$ amplitude was moderately reliable and similar to the reliability of the b-wave. PhNR amplitude measured from baseline was least reliable. A change in amplitude of around $50 \%$ in either PT or PF for an individual over two tests could be a marker of significant change in inner retinal function.

\section{PS B: Poster B14}

\section{Anthocyanins from black rice protect against retinal damage induced by intense light exposure}

\section{Lei Zhang, Qiang Cheng, Lin-song Qi, Zuo-ming Zhang}

\section{School of Aerospace Medicine, Fourth Military Medical University, Xi'an, China}

Retinal photic damage (RPD) is an important model for studying retinal regeneration in SD rats. Black rice anthocyanins (BRACs) are natural nutrients belonging to the flavonoid family which is famous for its antioxidant properties. In this paper we compared different administration of BRACs and the influence of different times on SD rats with light-induced RPD, showing that BRACs could protect the function and morphology of retinae over time. The results could be related to the suppression of oxidative stress and the degeneration of rhodopsins. We also identified that BRACs could pass through the blood-retina barrier to affect functions from an experimental aspect. Finally, the BRACs were evidenced by their protective function on the photic damaged retinae of $\mathrm{SD}$ rats. 


\section{Poster Session C}

\section{PS C: Poster C1}

\section{Thinking about trauma of lacrimal passages}

\section{Sha Zheng, Wei Qin, Chenxing Zhang, Qin Shu}

Southwest Eye Hospital, Southwest Hospital, the third Military Medical University, Chongqing, China

\section{Purpose}

To introduce some thinking about trauma of lacrimal passages. Methods

Literature review and clinical practice of our department.

\section{Results}

1. Treatment principles and skills of traumatic canaliculus fracture. i Reasons: sharp instrument injury, contusion injuries, foreign body injury. ii Location: superior canaliculus, inferior canaliculus, superior and inferior canaliculus. 2. Treatment principles of traumatic nasolacrimal canal injury. i Degree of complicating bone fracture. ii Complicating dacryocystisis. iii Complicating other lacrimal passages injuries. 3. Iatrogenic lacrimal passages injuries and its prevention. i Rinsing lacrimal passages from superior puncta is suggested to avoid excessive and inapposite rinsing. ii Bone fracture-caused nasolacrimal canal injury. iii Lacrimal sac injurycaused by inapposite usage of lacrimal passages timber. iv Canaliculus injury caused by inapposite lacrimal passages intubation. $\mathrm{v}$ Lacrimal sac and canaliculus injury caused by inapposite laser therapy. vi Lacrimal sac endometrium injury caused by inapposite operative procedure. Conclusions

It is important to normalize treatment of lacrimal passages injury.

\section{PS C: Poster C2}

\section{The preliminary study of intravitreal infusion in rabbit eyes}

\section{Tian Jiao, Luosheng Tang}

Department of Ophthalmology, Second Xiangya Hospital, Central South University, China

\section{Purpose}

The aim of this study was to explore the method of intravitreal infusion in rabbits, compare different ways of drug delivery, and research the effects of intravitreal infusion on retinal function and structure.

\section{Methods}

1. The preliminary design of intravitreal infusion: insulin pump was chosen as the infusion pump, the matched $27 \mathrm{G}$ needle was chosen and $0.06 \%$ trypan blue (TB) solution was taken for the infusion. 24 New Zealand rabbit were randomly divided into Group A, B, C, D with 6 eyes in each group. They were separately infused over 30 min with $50,100,150$ and $200 \mu 1$ of TB. Intraocular pressure (IOP) was measured at $0,5,10,15$, 20, 25 and $30 \mathrm{~min}$. The needle was pulled out and drug reflux, residual volume and eye changes were observed in each group
2. Comparison of different drug delivery methods: according to the results from part 1, Group B was taken as reference and designed Group E, F, G. 18 New Zealand rabbits were divided into 3 groups with 6 eyes in each group. Each group chose the $27 \mathrm{G}$ needle with $6 \mathrm{~mm}$ insertion. Group $\mathrm{E}$ underwent injection of $100 \mu \mathrm{l}$ of TB. Group F was bolus infused with $100 \mu \mathrm{l}$ of TB within $1 \mathrm{~min}$. Group $\mathrm{G}$ was infused with $100 \mu \mathrm{l}$ of TB over a period of $30 \mathrm{~min}$. Intraocular pressure (IOP), drug reflux, residual volume and eye changes in each group were observed and compared 3. To investigate the influence of different drug delivery methods for retinal function and structure, 42 New Zealand white rabbits were randomly divided into 7 groups. Each group included 6 eyes. The left eyes of group 1 were infused over $30 \mathrm{~min}$ with $100 \mu \mathrm{l}$ of balanced salt solution (BSS) with $6 \mathrm{~mm}$ insertion. The right eyes of group 1 underwent injection of $100 \mu \mathrm{l}$ of BSS. The left eyes of group 2 were bolus infused with $100 \mu \mathrm{l}$ of BSS within $1 \mathrm{~min}$. The right eyes of group 2 were normal control group. Treatments were performed 3 times on the first, fourth, and seventh days respectively. ERGs wereperformed before the treatment and $30 \mathrm{~min}$ after the first and third treatment. Eyes of experimental animals were enucleated on the seventh days and retinal structures were observed under optical microscope.

\section{Results}

1. The mean IOP during intravitreal infusion for groups A, B, C and $\mathrm{D}$ were $17.65 \pm 2.56,25.19 \pm 1.76,29.13 \pm 4.38$ and $39.77 \pm 8.54 \mathrm{mmHg}$, and the highest IOP during intravitreal infusion were $19.44 \pm 4.71, \quad 26.89 \pm 2.54, \quad 32.78 \pm 2.92$, $48.78 \pm 4.53 \mathrm{mmHg}$ respectively. In all four groups, TB was observed in the anterior chamber. Mild to moderate corneal edema with the increase of IOP was observed in some of group $\mathrm{C}$ and all of group D.2. The mean IOP post-injection for group E was $46.06 \pm 7.12 \mathrm{mmHg}$ and the residual volume was about $10-40 \mu \mathrm{l}$. The IOP values of group $\mathrm{F}$ after bolus infusion were more than $60 \mathrm{mmHg}$, but were less than $20 \mathrm{mmHg}$ after $30 \mathrm{~min}$. The mean IOP during intravitreal infusion for groups $\mathrm{G}$ was $25.27 \pm 1.85 \mathrm{mmHg}$, and lens damage near the puncture site occurred in two eyes 3. Each scotopic rod b-wave amplitude, scotopic maximal combined rod-cone b-wave amplitude and the [darkroom shock potential] amplitude value of ERG in each group before treatment, after the first treatment and after the third treatment results has no statistical difference $(P>0.05)$. Each retina tissue section did not show obvious abnormality under the optical microscope, the cells of each layers are complete and the structures are clear.

\section{PS C: Poster C3}

\section{MfERG with OCT after local retina laser coagulation on $\mathrm{C} 57 \mathrm{Bl} / 6$ mice}

\section{Skosyrski S, Dutescu M, Kociok N, Joussen A, Strauss O}

Department of Ophthalmology, Charite University Medizin, Berlin, Germany

\section{Purpose}

MfERG with OCT after local retina laser coagulation on C57Bl/6 mice. To test the feasibility of mfERG in conjunction with scanning-laser ophthalmoscope (SLO), OCT and 
fluorescence angiography (FA) in a laser coagulation model for mouse retinal degeneration.

\section{Conclusion}

The mfERG technique can be used to detect retinal damage as local changes in ERG function in mice. The usage of the mfERG technique in combination with OCT allows a correlation of defects in retinal function with structural alterations. It was possible to successively perform SLO fundus imaging, mfERG, OCT and angiography with the same animal.

Commercial relation: Roland Consult

\section{PS C: Poster C4}

\section{Bench top ERG \\ Sprehn G}

Rivendell Heights, Inc.Oceanside, CA, USA

\section{Purpose}

To record rat and mouse ERG in new laboratories.

\section{Methods}

Twenty-five new installations of bench top ERG systems were attempted over a period of two years, in six countries, with equipment designed for the purpose. In all cases, mice or rat models were anesthetized, dilated, dark-adapted, connected to electrodes, and stimulated with visible light. A dark environment was provided with local materials, ranging from cardboard and tape in a hallway to double-door wet labs.ERG stimulation was available from 0.01 to 50,000 candela, with flash duration of 1 to $10 \mathrm{~ms}$. Color filtration was provided. Ganzfeld as well as focal illumination of the retina was provided. General and localized retinal response was attempted.

\section{Results}

In all but one case, a measurable b-wave was achieved. In most cases, the a-wave was also measurable. Localized retinal response was confirmed in only a few cases. Reflection from the retina in a small mouse or rat eye caused most localized stimulation to result in a pseudo-Ganzfeld response. Ketamine-zylamine cocktail was superior to isofluorane or Avertin. Cataract formation was very fast in Jackson Labs C57BL/6 J and slow or non-existent in C57BL/ 6ByJ. Patience is essential. Dark is key; even pilot LEDs on wallmounted safety equipment was enough to suppress the b-wave. Direct stimulation through a contact fundus camera compared well to a Ganzfeld white bowl in a non-contact stimulator. Repeatability under stable conditions can be excellent.

\section{Conclusions}

With the right equipment, proper technique, and attention to detail, anyone can record and study bench-top ERG with small animals. Much can be gleaned from darkroom design of the days of silver-based film.

Gregory Sprehn is President of Rivendell Heights, Inc. and holds a financial interest.

\section{PS C: Poster C5}

\section{A live subretinal cysticerci in the eye}

\section{Xiaoyong Huang, Liu Hui}

Southwest Hospital/Southwest Eye Hospital, Third Military Medical University, Chongqing, China

A 31-year-old Dai woman was admitted to the hospital with a 2-month history of floaters with visual deterioration of right eye. There was history of consumption of ill-cooked pork. Visual acuity was 20/16 in left eye and 12/20 in right eye and could not be corrected. Binocular indirect ophthalmoscopy revealed mild yellow vitreous exudates with subretinal cyst in the upper nasal quadrant. This was further viewed with OCT. B-scan ultrasound showed a subretinal cysticercosis of right eye. Diagnosis included retinal detachment secondary to subretinal cysticercosis of right eye. Vitrectomy was performed successfully, the intact live subretinal cysticerci was removed with minimal invasiveness, and visual acuity of the right eye could be corrected to 20/20. Examination of the biopsy specimen showed that the cyst contained a Taenia solium cysticerci.

\section{PS C: Poster C6}

\section{A high-density EEG mapping study of texture-defined figure/ground segmentation in human visual development}

\section{Chuan Hou, Norcia AM}

Smith-Kettlewell Eye Research Institute, Stanford

University, San Francisco, CA, USA

\section{Purpose}

The visual system uses texture discontinuities and grouping mechanisms to segment visual scenes in objects and supporting background. Previous studies (Ales et al. 2013; Appelbaum et al. 2006) indicated that texture-defined figure/ground segmentation activate the visual area lateral occipital complex (LOC) which plays an important role in the perception of objects. The goal of this study was to use similar stimuli to investigate the development of extra-striate cortex, especially the area LOC.

\section{Methods}

We recorded 128-channel VEPs from 3-5 months old infants and from adults to the texture-defined figure/ground segmentation stimuli. We compared VEPs in two conditions: in a test condition, a $3 \times 3$ arrays of $4.5^{\circ}$ circular figures that appeared and disappeared from a uniform background at $1 \mathrm{~Hz}$. The figures and background $\left(24^{\circ} \times 24^{\circ}\right.$ visual field $)$ were composed of dynamic random bars that were updated at $15 \mathrm{~Hz}$. In a control condition, the texture within the circular figures also rotated between horizontal and vertical at $1 \mathrm{~Hz}$, but the circular figures remained segmented when the texture was horizontal with phase shift. A difference in response profile between these two conditions was used to isolate responses that estimate a pure segmentation of the stimulus.

\section{Results}

Infants showed a strong, but delayed segmentation-related responses compared to the adults: the initial segmentation response started at about $200 \mathrm{~ms}$ after the onset of the figures in infants versus 70-90 $\mathrm{ms}$ in adults. The infant response waveform was much simpler than that of the adults. Infant responses were restricted to the occipital midline at all latencies. The adults, by contrast, after an initial peak (70-90 ms) at the occipital pole, the 
adult response spread to lateral electrodes over right and left lateral cortex by $150 \mathrm{~ms}$. Activity over frontal electrodes occurred by $200 \mathrm{~ms}$, followed by later activity on lateral sites at $250-300 \mathrm{~ms}$. Conclusions

Infants at 3-5 months of age are sensitive to the global organization of our stimulus. However, they process texture segmentation cues in a restricted range of cortical areas. In particular, we found no evidence of activity in lateral occipital and frontal areas that is prominent in the adult evoked response.

\section{PS C: Poster C7}

Multiple evanescent white dot syndrome in a patient with history of acute transverse myelitis

\section{Jeeyun Ahn, Tae Wan Kim}

Department of Ophthalmology, Seoul Metropolitan Government Seoul National University Boramae Medical Center, Seoul, Korea

\section{Purpose}

To report a case of multiple evanescent white dot syndrome (MEWDS) in a patient with history of acute transverse myelitis who had been initially worked up for multiple sclerosis (MS) or neuromyelitis optica (NMO) with the suspicion of optic neuritis. Methods

A 26-year-old female patient with past history of acute transverse myelitis complained of sudden visual decline, photopsia and central scotoma in her right eye. Fundus examination of the right eye showed multiple white dots in the mid-peripheral retina and mild mottling of the retinal pigment epithelium in the macula. Initial differential diagnoses were retrobulbar optic neuritis, taking her past medical history into account, and MEWDS. After admission to the neurology department, full ophthalmological and neurological assessment, including perimetry, fluorescein angiography (FA), OCT, full-field and mfERG, and brain imaging was done.

\section{Results}

OCT showed disruption of the photoreceptor layer in the macula area and the blind spot was enlarged on perimetry examination. Mild red-green color defect was present and the P100 amplitude was decreased on VEP. Full-field ERG did not show any decrease in the a-wave amplitudes but the amplitudes of the first-order kernel of N1- and P1-wave amplitudes were supernormal in the macula area on mfERG. Initial brain MRI showed prominent enhancement of the retrobulbar fat and optic nerve sheath in both eyes but followup orbit MRI showed decreased nerve sheath enhancement taken one week later. The patient was subsequently diagnosed as MEWDS and discharged without undergoing steroid pulse therapy. Conclusion

Early MEWDS in a patient with past history of transverse myelitis may be misdiagnosed as retrobulbar optic neuritis. Detailed ophthalmologic examination is important for accurate differential diagnosis.

\section{PS C: Poster C8}

Full-field, macular, foveal pseudorandom ERGs: a comparative analysis
Shimada Y, Nakamura A, Tanikawa A, Horiguchi M

Department of Ophthalmology, Fujita Health University Banbuntane Hotokukai Hospital, Nagoya, Japan

\section{Purpose}

To investigate the functional characteristics in the whole retina, the macula and the fovea with pseudorandom electroretinograms (prERGs).

\section{Methods}

Five healthy trained subjects were recruited for mfERG and the full-field PERG (ffPERG) with $18.75 \mathrm{~Hz}$ base-rate and $2.67 \mathrm{cds} / \mathrm{m}^{2}$ stimulus intensity. MfERGs were recorded with isomeric 509 stimulus elements and $29 \mathrm{~min}$ recording time. The response at the centermost 61,7 and 1 elements corresponded to the macula $(<15$ degree in diameter), fovea $(<2.5 \mathrm{deg})$ and foveola $(<1.0 \mathrm{deg})$ respectively. ffPERGs were obtained with a built-in LED contact lens electrode, $1 \mathrm{~ms}$ stimulus duration and $1 \mathrm{~min}$ recording time.

\section{Results}

The first order kernel (K1) of the ffPERG, macular and foveal mfERGs had a positive component (PC) on the ascending limb of b-wave. PC was larger in the macula and the fovea. The first slice of the second order kernel (K2.1) in the foveola was gigantic and unique in shape with the prominent component with a long peak time around $50 \mathrm{~ms}$ (P50). The K1 of ffPERG was close to that of the summed mfERGs and $\mathrm{K} 2.1$ was very small as well as the summed mfERGs.

\section{Conclusions}

While ffPERG traced mfERGs obtained from the posterior pole including the macula and the fovea, unique responses evoked in the macula and the fovea contribute little to ffPERG. Particular K1 and prominent K2.1 provided from the fovea, probably reflect its expansive neural structures.

\section{PS C: Poster C9} Retinal function change of cynomolgus monkeys
with successful sub-retinal injection

Ming Mei, Jingyi Dai, Pinhong Rao, Laisheng Jiang, Yunyun Song

Joinn-Laboratories, Taicang, Jiangsu, China

\section{Purpose}

To evaluate the retina function change of cynomolgus monkeys with successful sub-retinal injection at various time points.

\section{Methods}

Six cynomolgus monkeys underwent sub-retinal injection of $75 \% \mathrm{NaCl}$ sodium or EGFP (50 or $100 \mu \mathrm{l})$. The injection was done under visual guidance of an operation microscope. Fullfield ISCEV ERGs were recorded at pre- (day1) and postinjection (day 2, day 7, and month 1). The ERG measurement includes dark-adapted 0.01 ERG, dark-adapted 3.0 ERG, darkadapted 3.0 OPs, dark-adapted 10.0 ERG, dark-adapted 10.0 OPs, light-adapted 3.0 ERG, and light-adapted 3.0 flicker ERG. The amplitudes and latencies of a- and b-waves of each measurement at each time point were analyzed. 


\section{Results}

Out of the twelve eyes of six monkeys, one sub-retinal injection failed. For the amplitude of a-waves, a non-significant reduction in right eyes was observed at day 2 and 7 compared to day- 1 and month 1; a non-significant reduction in left eyes was observed at month 1 than other time points. For the amplitude of $b$-waves, both eyes showed non-significant reductions at day 2 and 7 compared to day1 and month 1. For the implicit time, a non-significant delay of a-waves was observed at day 2 in both eyes, while a shorter but nonsignificant implicit time of b-waves was observed at day 2 and 7 . Conclusion

In general, retinal wound caused by sub-retinal injection recovered at around one month after the injection.

\section{PS C: Poster C10}

\section{Application of pattern VEPs in the perioperative period of orbital tumor patients}

\section{Wei Qin, Mingming Liu, Zheng Qin Yin}

Southwest Hospital/Southwest Eye Hospital, Third Military

Medical University, Chongqing, China

\section{Purpose}

The aim of the present study was to evaluate changes of the pattern VEP in perioperative orbital surgery patients.

\section{Methods}

Pattern VEPs, visual fields and CT or/and MRI were performed in 15 patients with orbital tumor before and after orbital surgery. Cavernous hemangioma, schwannoma, optical nerve glioma, meningioma, lymphoma and adenoid cystic carcinoma were 4,3,1,3,3 and 1 case respectively. Before and after the surgery, we analyzed the visual acuity, orbital pressure, pattern VEP, visual field and image findings.

\section{Results}

Patients with higher orbital pressure and longer history showed obvious changes of pattern VEP. When orbital apex or/and optic nerve were involved, the changes of pattern VEP became serious. Orbital apex or/and optic nerve were involved in cavernous hemangioma, schwannoma, optical nerve glioma, meningioma, lymphoma and adenoid cystic carcinoma were 2,1,1,3,0 and 0 cases respectively.

\section{Conclusions}

Pattern VEPs could help to evaluate objectively the risk of orbital surgery.

\section{PS C: Poster C11}

Clinical features of Sorsby fundus dystrophy and photodynamic therapy plus intravitreal anti-VEGF for macular choroidal neovascularisation

\section{Zhongshan Chen}

Department of Ophthalmology, Wuhan General Hospital of Guangzhou Military Command, Wuhan, China

\section{Purpose}

To investigate the clinical features of 6 cases (11 eyes) of Sorsby fundus dystrophy (SFD), and explore the efficacy of photodynamic therapy plus intravitreal injection anti-VEGF for secondary macular choroidal neovascularization $(\mathrm{CNV})$.

\section{Methods}

Retrospectively analyze the clinical data of 6 patients ( 6 eyes) of SFD with 3 male and 3 female, and mean age $38.5 \mathrm{yr}$. Fundus photography, fundus fluorescence angiography (FFA), indocyanine green angiography (ICG) and OCT were performed. Four cases were examined for gene mutations and the target gene was TIMP-3. Gene mutation analysis showed mutation of TIMP-3. Five eyes had macular CNV and these patients had undergone photodynamic therapy (PDT) plus intravitreal injection of Lucentis. The patients had best corrected visual acuity (BCVA), fundus photography, FFA, ICGA and OCT recorded before and after treatment.

\section{Results}

The BCVA increased comparing with before treatment. The regression of the $\mathrm{CNV}$ and resolution of the subretinal fluid were observed by FFA, ICGA and OCT after PDT plus IVB. The mean thickness of central retina was reduced greatly. There was no PDT associated ocular or systemic side effect. Conclusions

SFD has special clinical features, and it is difficult for diagnosis merely depending on the fundus appearances. PDT plus intravitreal injection anti-VEGF was an effective and safe treatment for SFD with CNV. It may improve or stabilize the visual acuity of SFD patients.

\section{PS C: Poster C12}

Visual electrophysiological findings in patients with liver cirrhosis and hepatic encephalopathy

Lei Zhang, Li Li, Xinmin Zhou, Feng Xia, Zuoming Zhang

School of Aerospace Medicine, Xijing Hospital, Fourth Military Medical University, Xi'an, China

Clinical observation of the neuropsychiatric condition, psychometric tests, and EEG recordings have been used to diagnose hepatic encephalopathy. However, no standardization for the diagnosis of minimal hepatic encephalopathy (MHE) has been achieved. According to the study in normal subjects, visual electrophysiology testing is a method of objective record for the medical examination, which includes VEP, ERG, etc. 23 hepatic encephalopathy patients prospectively underwent visual electrophysiology testing and laboratory serum analysis, and 22 visual electrophysiology abnormalities were found which showed that the amplitudes were decreased and the implicit times were prolonged compared with the normal. There was a correlation between biochemical parameters and visual electrophysiology parameters. On the basis of this preliminary study, maybe we could conclude that this method can be a way to predict for hepatic encephalopathy and it appears to be a method in the recognition of the MHE. 